\title{
Dark Matter-Neutrino Scattering in the Galactic Centre with IceCube
}

\author{
by \\ AdAm MCMullen \\ A thesis submitted to the \\ Department of Physics, Engineering Physics and Astronomy \\ in conformity with the requirements for \\ the degree of Master of Science \\ Queen's University \\ Kingston, Ontario, Canada \\ May 2021
}

Copyright (C) Adam McMullen, 2021 


\begin{abstract}
While there is evidence for the existence of dark matter, its properties have yet to be discovered. Simultaneously, the nature of high-energy astrophysical neutrinos detected by IceCube remains unresolved. If dark matter and neutrinos are coupled to each other, they could exhibit a non-zero elastic scattering cross section. Such an interaction between an isotropic extragalactic neutrino flux and dark matter would be concentrated in the Galactic Centre, where the dark matter column density is the greatest. This scattering would attenuate the flux of high-energy neutrinos, which could be observed in the IceCube South Pole Neutrino Observatory. In this thesis, an unbinned likelihood analysis is performed to set sensitivities for the seven year medium energy starting event cascades dataset for a signal considering possible dark matterneutrino interaction scenarios. This signal would be observed as a suppression of the high-energy astrophysical neutrino flux in the direction of the Galactic Centre. It is shown that IceCube can set constraints on possible dark matter-neutrino interactions that are complementary to constraints set by large scale structure surveys and the cosmic microwave background.
\end{abstract}




\section{Acknowledgments}

I would like to thank my supervisor Aaron Vincent at Queen's and co-supervisor Carlos Argüelles at Harvard for their support and guidance during the course of this thesis. I have been extremely lucky to have great supervisors who patiently answer all my questions and provide invaluable advice. I would also like to thank Austin Schneider for his excellent insight with this analysis. I would like to acknowledge the support of NSERC, the IceCube collaboration, the Frontenac Cluster, and the Canadian Armed Forces in making this thesis possible.

I would also like to thank all my family and friends for their support during this degree. I would especially like to thank Eric and Mikayla McMullen for editing this thesis and Simran Nerval for providing feedback for my thesis presentation. I would also like to thank the members of my thesis committee, Joe Bramante and Nahee Park for their comments and discussion during the thesis defence. 


\section{Contents}

Abstract $\quad$ i

Acknowledgments $\quad$ ii

Contents

List of Tables $\quad$ vi

List of Figures $\quad$ vii

Chapter 1: Introduction 1

Chapter 2: Dark Matter 4

2.1 Evidence of Dark Matter . . . . . . . . . . . . . . . . . . . 4

2.1.1 Velocity Dispersion . . . . . . . . . . . . . . . 4

2.1.2 Galactic Rotation Curves . . . . . . . . . . . . . 5

2.1.3 Cosmic Microwave Background . . . . . . . . . . . . . 6

2.1.4 Large Scale Structure . . . . . . . . . . . . . . . . . . . . . 9

2.2 Dark Matter Particle Candidates . . . . . . . . . . . . . . . 9

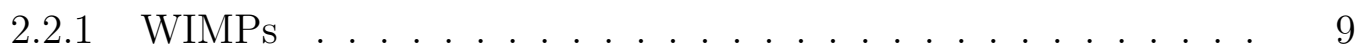

2.2 .2 Sterile Neutrino . . . . . . . . . . . . . . . . . 11

2.2.3 Axions ............................ 13

2.3 Dark Matter Density Profiles . . . . . . . . . . . . . 13

Chapter 3: Neutrinos and IceCube 16

3.1 Neutrinos . . . . . . . . . . . . . . . . . . . . . 17

3.2 Neutrino Sources . . . . . . . . . . . . . . . . . . . 17

3.3 Neutrino Production . . . . . . . . . . . . . . . . . . . . . . . 19

3.4 Neutrino Propagation . . . . . . . . . . . . . . . . . . 20

3.4.1 Extragalactic Neutrino Oscillation . . . . . . . . . . . . 20

3.4.2 Neutrino Attenuation in Matter . . . . . . . . . . . . . 23

3.5 Detecting Neutrinos . . . . . . . . . . . . . . . . 27 


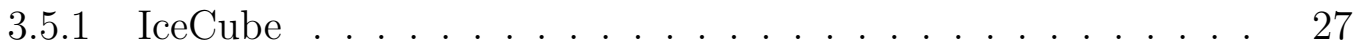

3.5.2 Event Morphologies . . . . . . . . . . . . . . . . . . . . . . . . 29

3.5.3 Distinguishing Astrophysical Events . . . . . . . . . . . . . 31

3.6 Modelling Neutrinos Observed at IceCube . . . . . . . . . . . . . . 34

3.6 .1 Astrophysical Sources . . . . . . . . . . . . . . . . . . . . 34

3.6 .2 Atmospheric Neutrinos . . . . . . . . . . . . . . . . . . . . 36

3.6.3 Atmospheric Muons . . . . . . . . . . . . . . . . . . . . . . . 39

Chapter 4: Neutrino-Dark Matter Interactions 40

4.1 Dark Matter Detection . . . . . . . . . . . . . . . . . . . 40

4.1 .1 Production at Colliders . . . . . . . . . . . . . . . . . . . 41

4.1 .2 Direct Detection . . . . . . . . . . . . . . . . . . 41

4.1 .3 Indirect Detection . . . . . . . . . . . . . . . . . . . . 42

$4.2 \quad$ Scattering . . . . . . . . . . . . . . . . . . . . 44

4.3 Dark Matter-Neutrino Scattering . . . . . . . . . . . . . . 44

4.3 .1 Previous HESE work . . . . . . . . . . . . . . . . . 46

4.3 .2 Cascade Equation . . . . . . . . . . . . . . . . . . . . 46

4.3 .3 Other Considerations . . . . . . . . . . . . . . . . . 47

4.3.4 Dark Matter-Neutrino Scattering Models . . . . . . . . . . 50

4.3.5 Dark Matter-Neutrino Interactions in Cosmology . . . . . . . 53

4.3.6 Comparing IceCube constraints with Cosmology . . . . . . . 56

$\begin{array}{lll}\text { Chapter 5: } & \text { Method } & 59\end{array}$

$5.1 \quad$ MESE-C Dataset . . . . . . . . . . . . . . . . . . . . . . . . . 59

5.1 .1 Monte Carlo Simulated Data . . . . . . . . . . . . . . . 63

5.2 Dark Matter Hypothesis . . . . . . . . . . . . . . . . . . . 65

5.3 Likelihood . . . . . . . . . . . . . . . . . . . . . . . 70

5.3.1 Kernel Density Estimation . . . . . . . . . . . . . . . . . 73

5.4 Markov Chain Monte Carlo . . . . . . . . . . . . . . . . 77

$\begin{array}{lll}\text { Chapter 6: } & \text { Sensitivity Analysis } & 80\end{array}$

6.1 Nuisance parameters . . . . . . . . . . . . . . . . . . . 80

6.2 Scalar DM-Scalar Mediator . . . . . . . . . . . . . . . 82

$\begin{array}{lll}\text { Chapter 7: } & \text { Conclusions and Future Work } & 87\end{array}$

7.1 Conclusion . . . . . . . . . . . . . . . . . . . . . 87

7.2 Future Work . . . . . . . . . . . . . . . . . . . . . . 88

$\begin{array}{ll}\text { Bibliography } & 90\end{array}$

$\begin{array}{ll}\text { Appendix A: Cross Sections } & 109\end{array}$ 
A.1 Matrix elements . . . . . . . . . . . . . . . . . 109 


\section{List of Tables}

5.1 Comparison of the HESE [1] and MESE-C [2] datasets. The number of each type of event are based on fits to models described in Sec. 3.6. 60 


\section{List of Figures}

2.1 Galactic rotation curves observed by Rubin and collaborators for seven spiral galaxies. These curves are compared with the no DM curve. Figure is modified from Ref: $[3] \ldots \ldots \ldots \ldots \ldots$

2.2 Angular power spectrum of the CMB. The large peaks correspond to acoustic oscillations, while the high multipole numbers are reduced by Silk damping. Fig from Ref: $[4] \ldots$. . . . . . . . . . . . . . .

2.3 The evolution of DM density is shown, where the comoving number of density of DM $Y=n / s$ is plotted over $x=m_{\chi} / T$, which can be taken as a proxy for time. $n$ is the number density, $s$ is the entropy density, $m_{\chi}$ is the DM mass and $T$ is the temperature.In the Early Universe (left) the temperature is high and DM annihilation and production to/from standard particles are in equilibrium. As the Universe expands and cools there is not enough energy for the Standard Model particles to produce DM, while the DM continues to annihilate. This leads to the number density of DM dropping exponentially. Eventually the Universe will have so much that the DM can no longer annihilate and the DM becomes constant (freeze out). The point at which freeze out occurs is dependent on the annihilation cross section $\langle\sigma v\rangle$. Figure from

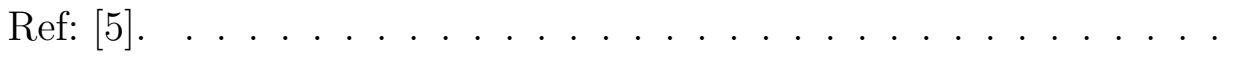


2.4 Popular DM density profiles. Most profiles are similar outside the orbit of the solar system ( $8.5 \mathrm{kpc}$ radius). These profiles are parameterized based on Ref: [6]. . . . . . . . . . . . . . . .

3.1 The grand unified neutrino spectrum shows the neutrino flux from a variety of sources across energy. Figure is taken from Ref: [7]. . . .

3.2 Triangle plot of flavour ratio observed in IceCube's 7.5 year high energy starting event sample. While the flavour ratio is not strongly constrained it is consistent with a source ratio of (1:2:0) which would indicate neutrino production from pion decay (red marker). The other coloured markers show different flavour ratios at neutrino production, that are all allowed. The best fit point is shown with the black star, and $1 \sigma$ and $2 \sigma$ contours are shown with black lines. Figure from Ref: [8]. .

3.3 Charged and neutral current interactions . . . . . . . . . . . .

3.4 Graphical layout of IceCube [9]. The detector is composed of 86 vertical strings suspended in the ice across a hexagonal grid with $125 \mathrm{~m}$ spacing. Each string contains 60 DOMs that are each separated by $17 \mathrm{~m}$. The DOM are buried from $1450 \mathrm{~m}$ to $2450 \mathrm{~m}$ below the ice's surface. IceCube is composed of a surface array, IceTop, and a denser inner subdetector, DeepCore, that significantly enhances the capabilities of the observatory, making it a multipurpose facility. DeepCore lowers the neutrino energy threshold to $10 \mathrm{GeV}[10]$. . . . . . . . .

3.5 Different event morphologies observed at IceCube from tracks, cascades or 'double bangs'. Figure from [11]. . . . . . . . . . . . . . . . . . . . 30

3.6 Veto technique . . . . . . . . . . . . . . . 33 
3.7 The veto region is used to remove an atmospheric neutrino that is coincident with a muon from the signal [9] . . . . . . . . . . . 34

3.8 The atmospheric neutrino flux at IceCube is dominated by conventional rather than prompt neutrinos. Figure from Ref: [12]. . . . . . . . . . 35

3.9 There are many different models to describe the astrophysical neutrino flux. Figure from Ref: $[12] . \ldots \ldots \ldots$

3.10 Best fits for the spectral index and flux normalization constant [13]. . 37

3.11 The cosmic ray air shower that leads to the production of the background atmospheric neutrinos observed at IceCube. The interactions shown are not complete and does not show the associated electromagnetic component and muon shower. Figure from Ref: [14]. . . . . . 38

4.1 Schematic diagram for the different methods of detecting DM. Indirect detection involve the production of Standard Model (SM) particles from dark matter $(\mathrm{DM})$. Direct detection involves the scattering of DM with SM. Collider techniques involve producing DM from SM. . .

4.2 The angular dependence of the differential cross section. Small scattering angles are strongly favoured indicating that after DM-neutrino scattering the neutrino maintains is original direction. . . . . . . . . 
4.3 A comparison of the extragalactic and galactic contributions to the DM column density. Here the distance to the neutrino source is the length from Earth over which the column density is integrated ( $x$ in Eq. (4.4)). The extragalactic component is modelled with the redshifted DM from (4.6), while the galactic component is modelled using the NFW halo described by (2.11). The line for the Galactic Centre represents the comoving distance to the Galactic Centre converted to a redshift. The contribution to dark matter column density from the Milky Way halo dominates up to redshifts $\sim 1$ where the contribution from relic dark matter outside the Milky Way. It can also be seen that the angles nearest to the galactic centre have higher column densities. $\quad 50$

4.4 DM-neutrino scattering models . . . . . . . . . . . . . . 51

4.5 DM-neutrino scattering cross sections . . . . . . . . . . 53

4.6 CMB angular power spectrum for varying effects of a neutrino-DM interaction cross section. Fig. from Ref: [15]. . . . . . . . . . . 55

5.1 Distribution of events in energy and declination . . . . . . . . . . 61

5.2 Distribution of events in energy and declination . . . . . . . . . . . 62

5.3 The angular resolution of the MESE-C dataset. The low energy neutrinos have a high angular error. Figure from Ref: [2] . . . . . . . . . 62

5.4 The different reconstruction methods that were used in [12]. These are only shown for the Monte Carlo simulation, where true is the declination that represents the neutrino energy at an astrophysical source. .

5.5 Skymap of the DM column density for a) a Burkert profile and b) an NFW profile. The column density increases at the Galactic Centre. . 
5.6 Skymap of the survival probability for the astrophysical neutrino flux. This is for the scalar mediator-scalar DM scenario with $g=1, m_{\phi}=10^{6}$ $\mathrm{MeV}, m_{\chi}=1 \mathrm{GeV}$ at $E_{\nu}=1 \mathrm{PeV} \ldots \ldots \ldots \ldots$

5.7 Survival probability for a neutrino flux across energy and angle to the Galactic Centre. This is for the scalar mediator-scalar DM scenario with $g=1, m_{\phi}=\mathrm{MeV}, m_{\chi}=1 \mathrm{GeV}$. The low energy $(\leq 20 \mathrm{TeV})$ part of the neutrino flux is completely attenuated by DM-neutrino scatter, while the high energy $(\leq 500 \mathrm{TeV})$ is unaffected. The inclusion of energies down to $1 \mathrm{TeV}$ by MESE-C compared to the $30 \mathrm{TeV}$ of HESE demonstrates how MESE-C will be able to better probe certain DMneutrino scattering interactions. . . . . . . . . . . . . .

5.8 The energy spectrum that includes a DM attenuation of the astrophysical neutrino flux. . . . . . . . . . . . . . . . . .

5.9 A comparison of kernel density estimation (KDE) and histogram binning. KDEs place a kernel at each event location and take the sum of the kernels to determine the overall probability density. . . . . . . . . 74

5.10 Probability density functions for the observable parameters . . . . . . 76

5.11 Different bandwidths and their effects on the resultant PDF. In this case the ideal bandwidth was about 0.1 and was determined using a chi squared figure of merit. . . . . . . . . . . . . . . .

6.1 Posterior probabilities as sampled by emcee for the no DM scenario. This was to ensure that reasonable values for the spectral indices and normalizations of the background models. . . . . . . . . . . . 
6.2 The posterior probability distribution for the DM parameters as sampled by emcee. ........................ 83

6.3 Best fit observables . . . . . . . . . . . . . . . . 83

6.4 The maximum coupling constant that is expected to be allowed by IceCube. Above the line constraints from IceCube are more stringent, while below the line those from cosmology dominate. . . . . . . . .

6.5 The upper limit on the sensitivity for a neutrino-DM cross section is shown in black across DM mass. Also plotted are the DM-electron and DM-nucleon scattering cross section limits from SENSEI, XENON1T and XENON10 as presented in Ref: [16]. Since the cross section is energy dependent, this is plotted for a neutrino energy of $46 \mathrm{TeV}$. . .

A.1 Feynman diagram of scalar DM-scalar mediator scattering with momenta labels. . . . . . . . . . . . . . . . . . . . . . . 110 


\section{Chapter 1}

\section{Introduction}

One of the greatest problems in astrophysics is in determining what composes the Universe. To date, the best understanding of what makes up the Universe can be extracted from Planck observations of the cosmic microwave background (CMB), which indicate that $5 \%$ of the Universe is regular matter, while the rest is dark matter (DM) (26\%) and dark energy (69\%) [17]. Whereas the Standard Model of particle physics allows one to reasonably understand the $5 \%$ of regular matter, the nature of the majority of matter remains unresolved.

Over the last century the presence of DM has been implied by numerous observations of its gravitational effects, however, it has yet to be detected. Various theories have been developed to describe DM as a particle including weakly interacting massive particles (WIMPs), axions, and sterile neutrinos [18]. Experiments to search for these models of DM have thus far proven fruitless (there have been some reports of DM detection, but none that have been verified). Despite this lack of a signal, some information about DM can be gleaned, by ruling out various theorized models and constraining parameters. These searches usually involve searching for possible interactions between DM and Standard Model particles and are typically directed towards 
areas where a large signal can be expected, such as the Galactic Centre.

The IceCube Neutrino Observatory is a $1 \mathrm{~km}^{3}$ ice Cerenkov detector located at the geographic South Pole. The detection of high energy (TeV-PeV) neutrinos of astrophysical origin in 2013 marked a key turning point in the history of multimessenger astronomy [19]. These neutrinos are believed to have originated in the high energy astrophysical sources outside the Milky Way, like active galactic nuclei (AGNs) [20,21]. In addition to supplementing the information from electromagnetic astronomy, the observation of high energy neutrinos also provides a novel means through which to probe the properties of DM [22].

As most past searches for DM have been directed at DM interactions with quarks or electrons, DM-neutrino interactions are one of the least explored (and constrained) connections of DM with the Standard Model. In certain DM models, neutrinos could elastically scatter with DM particles. This interaction would be concentrated in the Galactic Centre where the DM density is highest and would lead to an energy dependent shadow in the astrophysical neutrino flux in the direction of the Galactic Centre that could be observed by IceCube [1]. The goal of this thesis is to perform a sensitivity analysis for IceCube, so that future analyses can place constraints on the properties of DM. Through an unbinned likelihood analysis searching for a shadow in the high energy neutrino flux at the Galactic Centre,sensitivities are obtained for a model of DM-neutrino scattering. This model is particular to a sub-GeV scale DM that can scatter with neutrinos. It will be shown that the neutrino observations from IceCube can be used to probe areas of parameter space unreachable by cosmology.

This thesis is organized as follows. Chapter 2 reviews the evidence and possible candidates for DM as well as the different density models used to describe the DM 
in the Milky Way. Chapter 3 provides an overview of IceCube and the high energy neutrinos it observes. Chapter 4 delineates the DM-neutrino interaction mechanisms in the context of cosmology and particle physics. Chapter 5 explains the Bayesian likelihood method that was used. Chapter 6 gives the results of the analysis and the sensitivities that can be achieved with IceCube. Chapter 7 concludes this thesis with a discussion on future work. 


\section{Chapter 2}

\section{Dark Matter}

\section{$2.1 \quad$ Evidence of Dark Matter}

Despite not having been directly detected, DM comprises $85 \%$ of the matter in the Universe [17]. The existence of DM is well motivated by the observation of its gravitational effects across multiple scales of astronomy and cosmology including: the cosmic microwave background (CMB), large scale structure, galactic rotation curves, collisions between galaxies, and gravitational lensing [23].

\subsubsection{Velocity Dispersion}

The effects of DM were first hinted at in 1933 when Fritz Zwicky noticed that the galaxies in the Coma cluster had a large scatter across the velocities [24]. By applying the virial theorem to estimate the total mass of the galaxies and comparing this with an estimate based on the average mass of visible galaxies, he measured a mass-tolight ratio of about 500 [25]. While this mass-to-light ratio was later refined, his conclusion remained that same: that there existed a missing 'dark matter' that was more abundant than luminous matter [23]. 


\subsection{EVIDENCE OF DARK MATTER}

\subsubsection{Galactic Rotation Curves}

The next major advancement providing evidence for DM came in the 1970's when Vera Rubin and others discovered that the galactic rotation curves also indicated there was missing matter [23]. This was done with observations of the velocity of visible stars and gas for various radial distances from the galaxy's centre. These rotation curves indicated that galaxies were rotating too quickly to agree with the orbital mechanics of only visible matter, signifying the presence of a dark matter [26].

For stars orbiting around the centre of a galaxy, the centripetal and gravitational forces will be equal allowing the orbital velocity to calculated:

$$
v=\sqrt{\frac{G M(r)}{r}},
$$

where $M(r)$ is the mass enclosed within an orbit at radius $r$ and $G$ is the gravitational constant [18]. By measuring the velocities of stars, the total mass contained within a galaxy can be determined with Eq. (2.1). Since the luminous mass appears concentrated in the Galactic Centre, the enclosed mass would be expected to remain constant at large radii. From Eq. (2.1) this would lead to the expectation that velocity would drop off at large radii as $r^{-1 / 2}[18]$.

Rubin and collaborators continued to observe galactic rotation curves using spectrographs, and found that the rotation curves did not fall off as expected at large radii [26]. An example of this is shown in Fig. 2.1. Alongside numerous other researchers, galactic rotation curves provide evidence that either there existed some unseen (dark) matter or the gravitational model was incorrect [18]. 


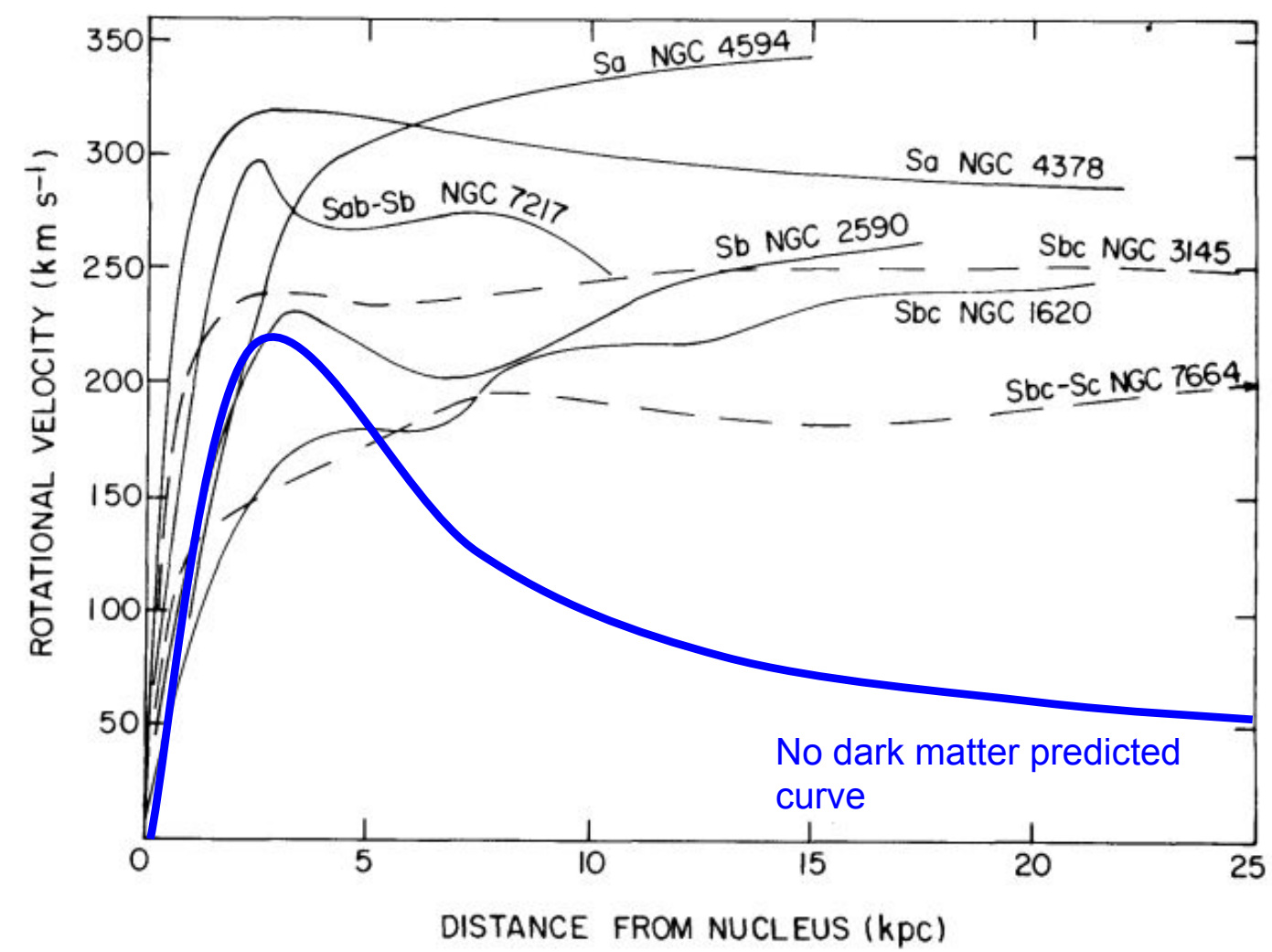

Figure 2.1: Galactic rotation curves observed by Rubin and collaborators for seven spiral galaxies. These curves are compared with the no DM curve. Figure is modified from Ref: [3].

\subsubsection{Cosmic Microwave Background}

Evidence for the existence of DM is also found in the observations of the cosmic microwave background (CMB). In the Early Universe, ordinary matter was ionized and interacted with radiation through Thomson scattering. About 370000 years after the Big Bang, the Universe had expanded and cooled enough for electrons and protons to form hydrogen in what is known in recombination [27]. At this point photons were able to freely stream. These photons mark the earliest signals that can 


\subsection{EVIDENCE OF DARK MATTER}

be observed, and are referred to as the cosmic microwave background. Observations of these photons can be used to determine the temperature field at recombination [27]. In particular, it is the deviation in the temperature that is important [28]:

$$
\Theta(\theta, \phi)=\frac{T(\theta, \phi)-\langle T\rangle}{\langle T\rangle}
$$

where $\Theta$ is the deviation from the average temperature at a location $(\theta, \phi)$ in the sky. This can be rewritten with spherical harmonics as [28]:

$$
\Theta(\theta, \phi)=\sum_{l=0}^{l=\infty} \sum_{m=-l}^{m=l} a_{l m} Y_{l m}(\theta, \phi)
$$

where $Y_{l m}$ is the spherical harmonic function with modes $m$ and $l$, and $a_{l m}$ is a coefficient given by: $a_{l m}=\int_{\theta=0}^{2 \pi} \int_{\phi=0}^{2 \pi} \Theta(\theta, \phi) Y_{l m}^{*} d \theta d \phi$. The power spectrum (Fourier transform) of these temperature fluctuations, $C_{l}$, is given as [28]:

$$
\left\langle a_{l m} a_{l^{\prime} m^{\prime}}^{*}\right\rangle=\delta_{l l^{\prime}} \delta_{m m^{\prime}} C_{l}
$$

Assuming an isotropic sky and assuming no correlations between modes, the two point temperature field is described by $[4,28]$ :

$$
C_{l}=\frac{1}{2 l+1} \sum_{m=-l}^{l}\left\langle\left|a_{l m}\right|^{2}\right\rangle .
$$

These temperature fluctuation are usually plotted as $\frac{l(l+1)}{2 \pi} C_{l}$ vs. the multipole mode $l[29]$ as shown in Fig. 2.2.

The peaks in this power spectrum are determined by the interactions in the Early 


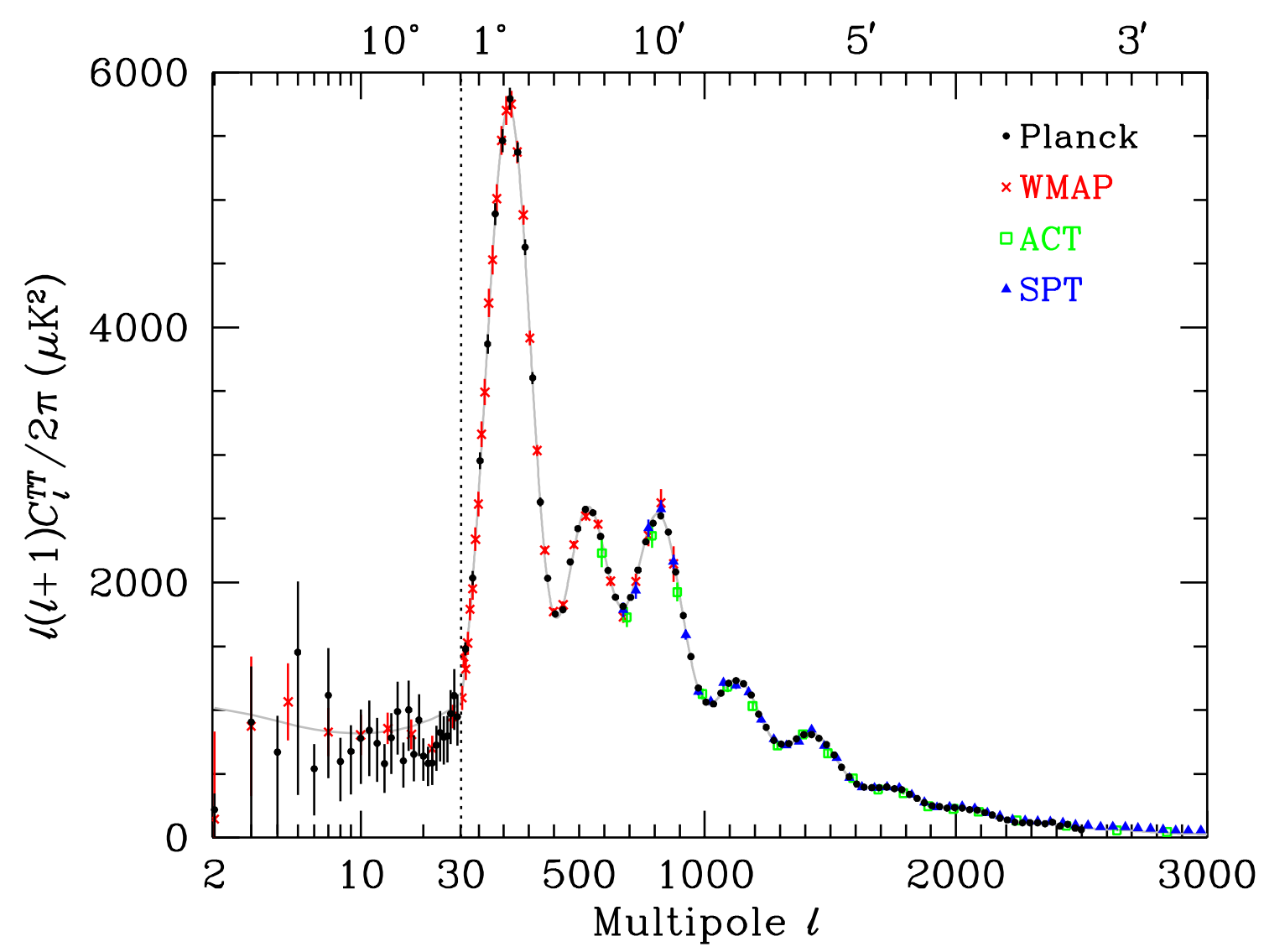

Figure 2.2: Angular power spectrum of the CMB. The large peaks correspond to acoustic oscillations, while the high multipole numbers are reduced by Silk damping. Fig from Ref: [4].

Universe. In the low multipole region of the power spectrum $1 \lesssim l \lesssim 100$ the SachsWolf effect is the dominant perturbation. Here perturbations in the gravitational potential $\delta \Phi$ can affect the temperature field through:

$$
\Theta_{\text {grav }}=\frac{1}{3} \frac{\delta \Phi}{c^{2}}
$$

At higher multipoles, the acoustic oscillations determine the magnitude and location of the peaks. These oscillations are the result of the counteracting forces of 


\subsection{DARK MATTER PARTICLE CANDIDATES}

gravitational attraction by dark and baryonic matter and the outward pressure from photon-matter interactions. These oscillations are analogous to sound waves hence the term acoustic. The peaks from acoustic oscillations are dependent on the amount of matter, with the second peak being constrained by the baryon density, while the third peak is constrained by the DM density. At high multipole numbers $(l \gtrsim 1000)$ Silk damping becomes dominant. This damping is caused by diffusing photons that travel from hot regions to cold regions and equalize the temperatures [4].

\subsubsection{Large Scale Structure}

On the large scales of the Universe, structures are formed due to the density perturbations in the Early Universe. These density perturbations collapsed to form stars, galaxies and the cosmic web [30]. N-body simulations of large scale structure are sensitive to the presence of DM and can be used to determine the relic DM density. While baryonic matter alone is washed out through constant interactions with the dominant radiation, DM does not interact with radiation and can is allowed to condense into structures [18].

\subsection{Dark Matter Particle Candidates}

There exists a myriad of models that have been theorized to describe DM. In the $\Lambda \mathrm{CDM}$ model DM is cold, collisionless and long-lived [31].

\subsubsection{WIMPs}

Weakly interacting massive particles (WIMPs) are a popular DM candidate that are massive ( $\mathrm{GeV}$ scale) and interact through the weak force or a simple weak coupling. 


\subsection{DARK MATTER PARTICLE CANDIDATES}

WIMPs are motivated by the WIMP miracle, where the annihilation of $\mathrm{GeV}$ weakly interacting DM particles to Standard Model particles can lead to the correct present abundance of DM [31].

\section{WIMP Miracle}

In the Early Universe, when the temperature was high, DM production and self annihilation were in equilibrium. However, as the Universe expanded and cooled there was not enough energy for SM particles to form DM particles. At this point, DM annihilation continued while DM production stopped, leading to the DM density falling exponentially [5]. After the universe further expanded, the DM density became so low that the DM particles could no longer annihilate. The DM density then becomes constant and is said to freeze out. This happens when [5]:

$$
n_{\chi}\langle\sigma v\rangle=H,
$$

where $n_{\chi}$ is the number density of $\mathrm{DM},\langle\sigma v\rangle$ is the thermally averaged annihilation cross section and $H$ is the Hubble parameter.

The self annihilation cross section that leads to the proper relic abundance of DM can be found by solving the Boltzmann collision equation. With the Boltzmann collision equation the number density of $\mathrm{DM}, n$ can be found as:

$$
\frac{d n}{d t}+3 H n=-\langle\sigma v\rangle\left(n^{2}-n_{e q}^{2}\right),
$$

where $\langle\sigma v\rangle$ is the thermally averaged annihilation cross section, $H$ is the Hubble 


\subsection{DARK MATTER PARTICLE CANDIDATES}

constant and $n_{e q}$ is the number density at thermal equilibrium [18]. In the nonrelativistic limit $n_{e q}$ becomes:

$$
n_{e q}=g\left(\frac{m T}{2 \pi}\right)^{3 / 2} e^{-m / T}
$$

where $g$ is the degrees of freedom, $m$ is the mass of the particle and $T$ is the temperature of the plasma.

After expanding in powers of $v^{2}$, the relic density parameter can eventually be approximated as [18]:

$$
\Omega_{\chi} h^{2} \approx \frac{3 \times 10^{-27} \mathrm{~cm}^{3} \mathrm{~s}^{-1}}{\langle\sigma \cdot v\rangle} .
$$

For a relic density of $h^{2} \Omega_{\chi}=0.12$, the cross section is $\langle\sigma v\rangle \sim 3 \times 10^{-26} \mathrm{~cm}^{3} \mathrm{~s}^{-1}$. This cross section is consistent with a $100 \mathrm{GeV}$ mass particle that interacts via the weak force. This is what is known as the WIMP miracle [5]. The evolution of the DM number density is shown in Fig. 2.3, where it can be seen that varying the the self annihilation cross section will result in different relic abundances of DM.

\subsubsection{Sterile Neutrino}

Sterile neutrinos are a hypothesized fourth flavour of neutrinos that do not interact through the electroweak force [32]. They only interact with the Standard Model through neutrino flavour mixing. Sterile neutrinos have been constrained by cosmol-

ogy to have a mass $m \lesssim 10 \mathrm{keV}$ [18]. IceCube has searched for a matter enhanced oscillation that would occur for light sterile neutrinos. They found no evidence of a fourth neutrino and constrained the mixing matrix elements $[33,34]$. 


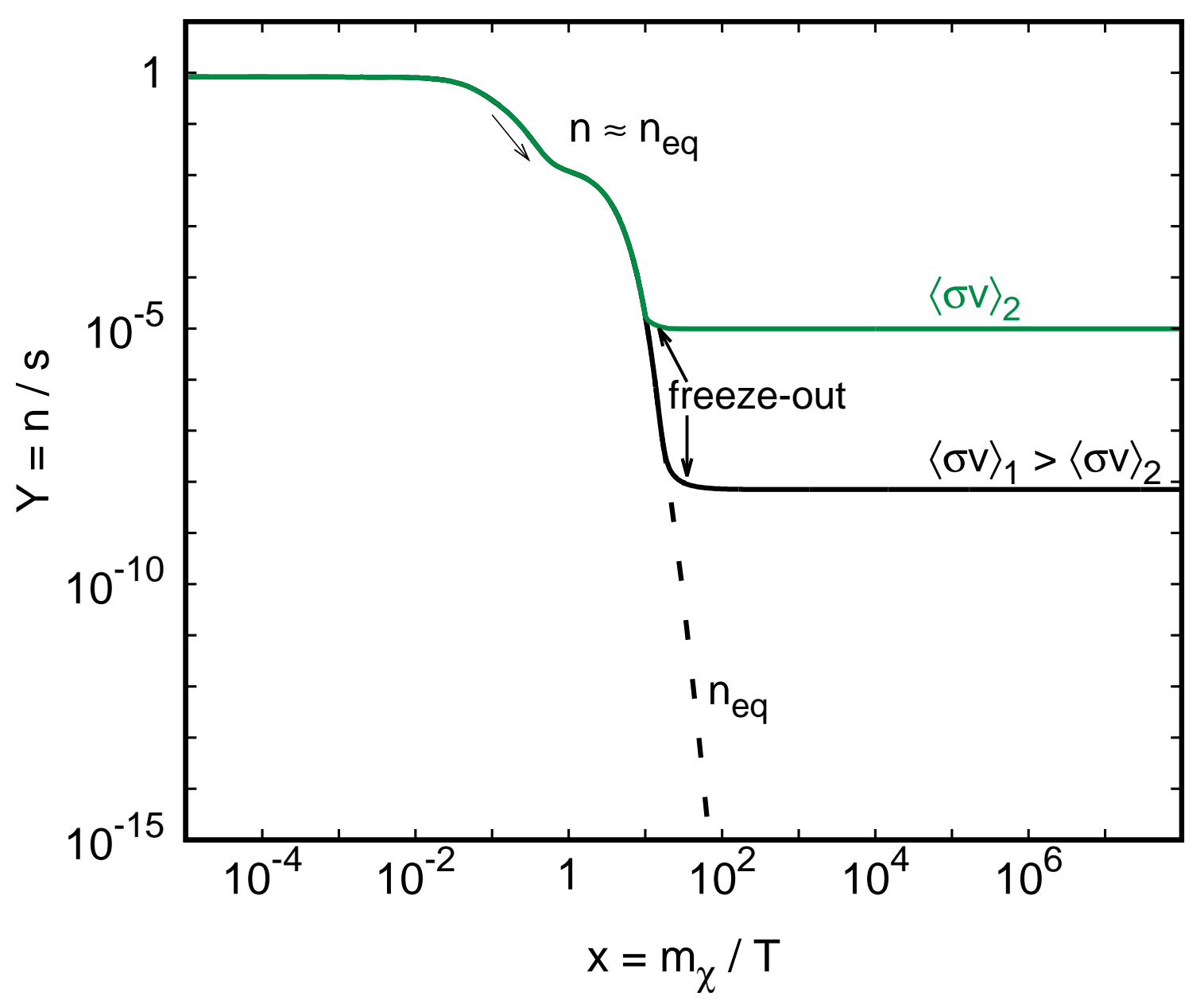

Figure 2.3: The evolution of DM density is shown, where the comoving number of density of DM $Y=n / s$ is plotted over $x=m_{\chi} / T$, which can be taken as a proxy for time. $n$ is the number density, $s$ is the entropy density, $m_{\chi}$ is the DM mass and $T$ is the temperature.In the Early Universe (left) the temperature is high and DM annihilation and production to/from standard particles are in equilibrium. As the Universe expands and cools there is not enough energy for the Standard Model particles to produce DM, while the DM continues to annihilate. This leads to the number density of DM dropping exponentially. Eventually the Universe will have so much that the DM can no longer annihilate and the DM becomes constant (freeze out). The point at which freeze out occurs is dependent on the annihilation cross section $\langle\sigma v\rangle$. Figure from Ref: [5]. 


\subsection{DARK MATTER DENSITY PROFILES}

\subsubsection{Axions}

Axions were introduced to solve the strong $\mathrm{CP}$ problem and have been considered as a DM candidate. Axions are constrained to be very light $\lesssim 10^{-2} \mathrm{eV}$. They are also very weakly interacting with normal matter [18].

\subsection{Dark Matter Density Profiles}

Numerous models to describe the DM density distribution have been proposed to agree with N-body simulations including the the Einasto, NFW, Burkert, and isothermal profiles. These density distributions are important for this thesis, as the number of DM particles with which neutrinos interact is required to determine the attenuation in neutrino energy. These distribution are shown in Fig. 2.4.

DM density profiles are generically parameterized with a double power law as shown with the Zhao profile [35]:

$$
\rho_{D M}(r)=\frac{\rho_{0}}{\left(\delta+\frac{r}{r_{s}}\right)^{\gamma}\left[1+\left(\frac{r}{r_{s}}\right)^{\alpha}\right]^{(\beta-\gamma) / \alpha}},
$$

for DM density $\rho$ at radius $r$, where parameters $\alpha, \beta, \gamma, \delta$ describe the shape of the halo, $\rho_{0}$ is the local DM density, and $r_{s}$ is the scale radius of the halo.

The Navarro-Frenk-White (NFW) profile is described with parameters $(\alpha, \beta, \gamma, \delta)$ of $(1,3,1,0) \quad[36]$ and the Burkert profile has parameters $(2,3,1,1)$ [37].

The Einasto profile is [38]:

$$
\rho(r)=\rho_{0} \exp \left(-\frac{2}{\alpha}\left[\left(\frac{r}{R_{s}}\right)^{\alpha}-1\right]\right) .
$$




\subsection{DARK MATTER DENSITY PROFILES}

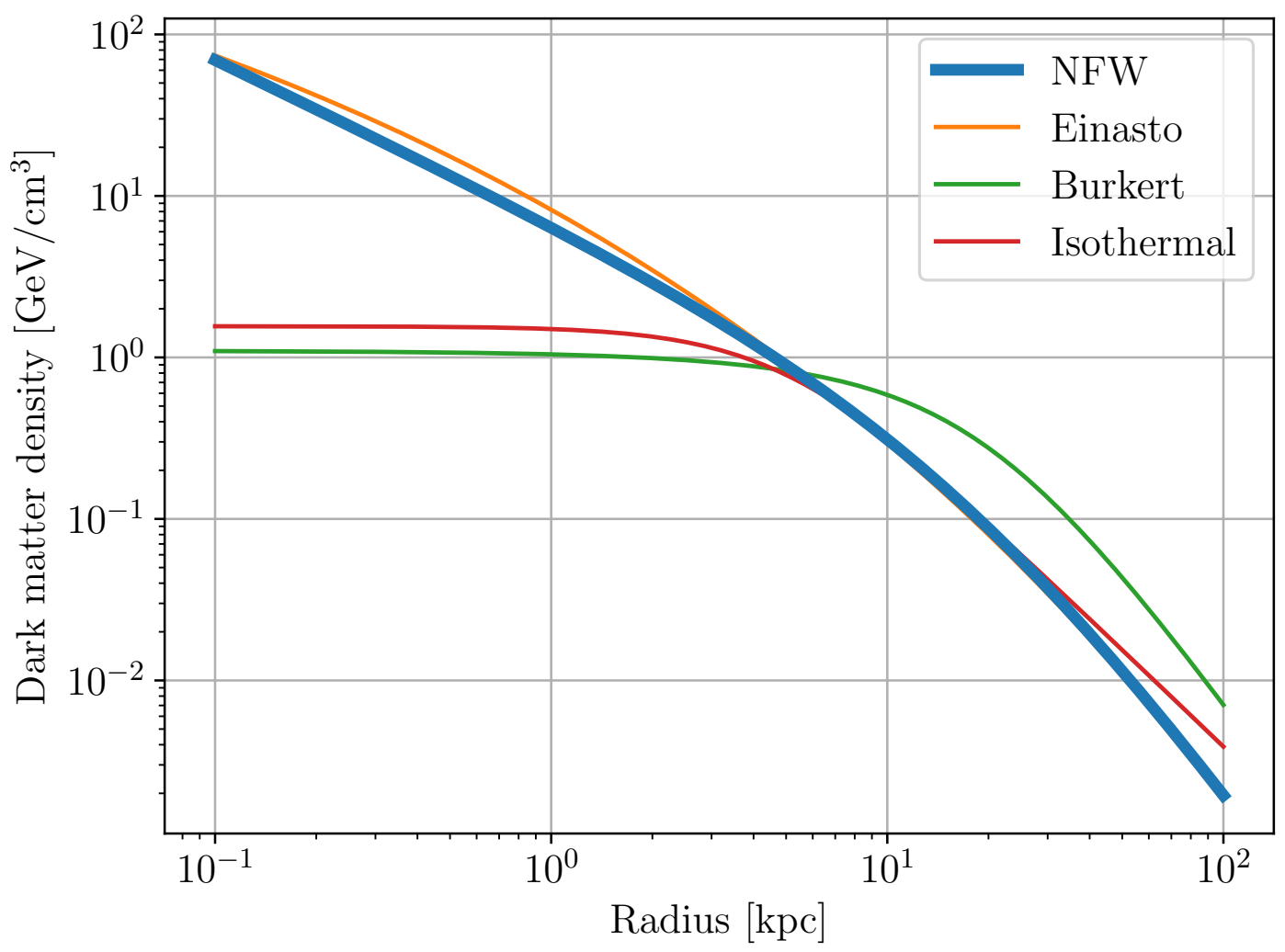

Figure 2.4: Popular DM density profiles. Most profiles are similar outside the orbit of the solar system ( $8.5 \mathrm{kpc}$ radius). These profiles are parameterized based on Ref: [6].

The DM profile that was used in this analysis was the NFW profile. The parameters that were used have been constrained by Benito et al in Ref: [39] and updated in Ref: [40]. They placed limits on the profile density and scale radius using data from Gaia and GRAVITY. The local DM density has $2 \sigma$ limits between $0.3 \leq \rho_{0} \leq 0.8 \mathrm{GeV} / \mathrm{cm}^{3}$. They find that the often used local DM density $\rho_{0}=0.3$ $\mathrm{GeV}$ and local distance to the Galactic Centre $R_{0}=8.5 \mathrm{kpc}$ is excluded by more than $4 \sigma$. They also found that there was no preference for a cored versus cuspy profile. In this thesis the following parameters are used: a scale radius of $R_{s}=26 \mathrm{kpc}$, scale 


\subsection{DARK MATTER DENSITY PROFILES}

density $\rho_{0}=0.4 \mathrm{GeV} / \mathrm{cm}^{-3}$, and distance to the Galactic Centre $R_{\text {sun }}=8.178 \mathrm{kpc}$.

Numerous observations in the past century have found evidence for DM, and many models have been theorized to describe it. While its true nature has yet to be determined, it is known to compose most of the matter in the Universe and form halos around galaxies. As one of the least explored interaction channels DM neutrino interactions could potentially occur in the DM halo of the Milky Way for which a signal could be detected in the IceCube Neutrino Observatory. The neutrino signal detected at IceCube will be explored in the next chapter. 


\section{Chapter 3}

\section{Neutrinos and IceCube}

Generally, cosmic ray research aims to answer three complementary questions: where do cosmic rays come from, how are they energized, and how do they propagate. Multimessenger astronomy can be used to answer these questions by analyzing data from gamma rays, gravitational waves, charged particles, and neutrinos. Here, data from each of the different 'messengers' provide different insights into astrophysical processes. As neutrinos are one of the most weakly interacting elementary particles in the Standard Model, they travel from their source to Earth without attenuation and without deflection by magnetic fields. They are also one of the most abundant particles in the Universe and may serve as an ideal probe of extra-galactic astrophysics. This weak interaction also makes them difficult to detect, as they require massive detectors, like IceCube, to obtain a statistically significant signal [41]. This chapter

describes the neutrinos that are detected at IceCube, which will be an important part of the analysis technique in Ch. 5. 


\subsection{NEUTRINOS}

\subsection{Neutrinos}

Neutrinos were theorized in 1930s by Wolfgang Pauli to account for the missing mass in beta decays. Enrico Fermi then coined the name neutrino or 'little neutral one'. Neutrinos were first observed in 1956 by Reines and Cowan with their underground reactor experiment [42]. Neutrinos are one of the most abundant particles in the Standard Model both in the Early Universe and today. They are predicted to have a small non-zero mass $(\lesssim 1 \mathrm{eV})$ [43], be electrically neutral, [4] and oscillate between flavours [44]. There are three flavours of neutrinos: electron, muon, and tau.

\subsection{Neutrino Sources}

Neutrinos are produced from a variety of sources across a wide energy range as shown in Fig. 3.1 [7]. They can come from cosmic rays scattering with CMB photons, astrophysical sources, atmospheric cosmic ray showers, nuclear reactors, the Sun, and the Earth [7]. At the lowest end of the energy spectrum, the neutrinos that come from the cosmic neutrino background $(\mathrm{C} \nu \mathrm{B})$ and from big bang nucleosynthesis $(\mathrm{BBN})$ have yet to be detected due to the high energy resolution that would be required to detect them. At the highest energy part of the spectrum, cosmogenic neutrinos have yet to be detected due to their low flux and significant background [45]. Since IceCube is sensitive to neutrinos in the energy range of $\mathrm{GeV}$ to $\mathrm{PeV}$, the neutrinos that IceCube observes are from atmospheric and astrophysical sources. In this case atmospheric neutrinos dominate the flux in the low energies and need to be modelled as a background, while the DM-neutrino signal will be incorporated in the model of the astrophysical neutrinos.

It is predicted that astrophysical neutrinos are produced in the highest energy 


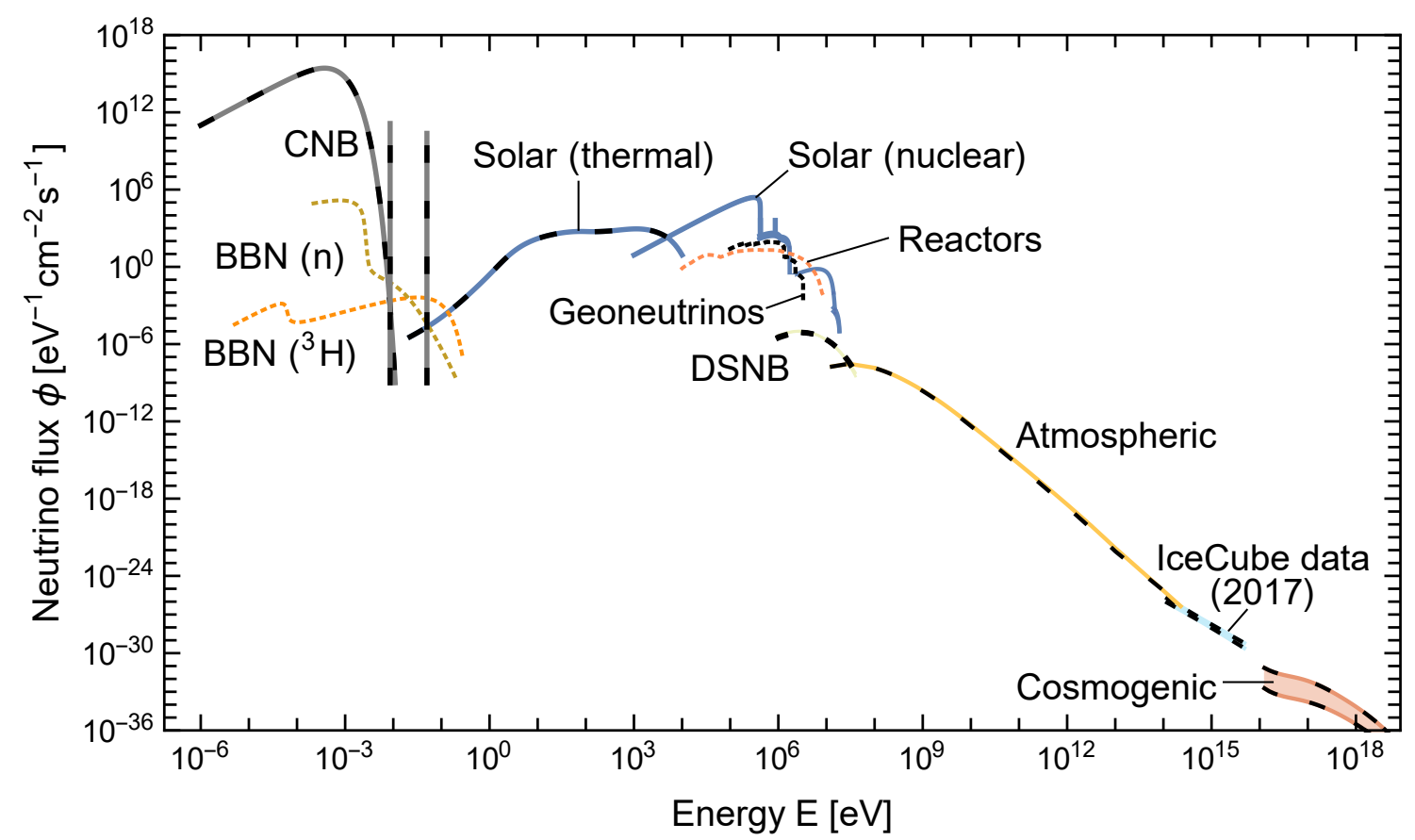

Figure 3.1: The grand unified neutrino spectrum shows the neutrino flux from a variety of sources across energy. Figure is taken from Ref: [7].

extragalactic sources such as the super-massive black holes at the centre of active galactic nuclei $[46,47]$, the collapse of massive stars in gamma ray bursts $[48,49]$, or in star burst galaxies [50]. Although some potential points sources have been identified and there is growing evidence for the blazar connection [51,52], the exact nature of astrophysical neutrino sources remain uncertain [53-62].

IceCube, which detects neutrinos in the $\mathrm{TeV}$ to $\mathrm{EeV}$ range $\left(10^{12}-10^{18} \mathrm{eV}\right)$, is dominated by atmospheric neutrinos and atmospheric muons. Atmospheric neutrinos are produced when cosmic rays interact with the Earth's atmosphere to produce mesons. In 2013, IceCube detected an excess of neutrinos above $100 \mathrm{TeV}$ that could not be explained by atmospheric sources. This excess was consistent with an an isotropic distribution and hinted to an extragalactic origin. This marked a key turning 


\subsection{NEUTRINO PRODUCTION}

point in the history of multi-messenger astronomy [19]. These neutrinos are posited to have originated in the highest energy astrophysical sources outside the Milky Way like AGNs. The astrophysical nature of these high energy neutrinos was further confirmed in 2018 when IceCube along with the gamma-ray telescopes Fermi-LAT and MAGIC announced the simultaneous detection of neutrinos and photons from blazar TXS 0506+056. This observation of neutrino and gamma-ray emission from TXS 0506+06 is the first evidence that blazars can produce the most energetic neutrinos and cosmic rays $[20,21]$.

\subsection{Neutrino Production}

Most models for astrophysical neutrino production have neutrinos as the byproducts of cosmic ray interactions with gas and radiation via hadronic $(p p, p n)$, and photohadronic $(\gamma p, \gamma n)$ interactions. In these interactions, protons that have been accelerated produce unstable mesons such as charged pions, kaons and D mesons that subsequently decay into neutrinos of all flavours [63]. The dominant $p p$ and $p \gamma$ interactions are summarized as:

$$
\begin{gathered}
p+p \rightarrow N\left[\pi^{0}+\pi^{+}+\pi^{-}\right]+X, \\
p+\gamma \rightarrow \Delta^{+} \rightarrow\left\{\begin{array}{l}
n+\pi^{+}, \\
p+\pi^{0} .
\end{array}\right.
\end{gathered}
$$

where $p$ is a proton, $\gamma$ is a photon, $N$ is a constant, $\pi^{ \pm}$are charged pions, $\pi^{0}$ are neutral pions and $X$ represents other cosmic ray particles that are produced alongside pions [64]. These interactions occur while the protons are accelerated, while they exit 


\subsection{NEUTRINO PROPAGATION}

their source and while they travel to Earth and involve transferring energies up to $5 \%$ of the energy of progenitor protons or nuclei to neutrinos. The pions produced through the mechanism of Eq. (3.1) subsequently decay through the dominant channel to muons [64]:

$$
\begin{aligned}
& \pi^{+} \rightarrow \mu^{+}+\nu_{\mu}, \\
& \pi^{-} \rightarrow \mu^{-}+\bar{\nu}_{\mu} .
\end{aligned}
$$

The muon can then decay into:

$$
\begin{aligned}
& \mu^{+} \rightarrow e^{+}+\nu_{e}+\bar{\nu}_{\mu}, \\
& \mu^{-} \rightarrow e^{-}+\bar{\nu}_{e}+\nu_{\mu},
\end{aligned}
$$

These neutrinos can then travel long distances without interacting to eventually be detected on Earth.

\subsection{Neutrino Propagation}

Due to the low average interstellar matter density, neutrinos can be expected to propagate through space without significant attenuation or deflection. This means that neutrinos observed on Earth can be expected to have retained their source energy and direction.

\subsubsection{Extragalactic Neutrino Oscillation}

While neutrino oscillation is an important factor to be accounted when observing neutrinos from nearby sources (like the Sun), flavour effects from astrophysical neutrinos can be shown to average out over extragalactic scales. In the case that the charged

pion and subsequent muon decay are the dominant processes at astrophysical sources, 


\subsection{NEUTRINO PROPAGATION}

then Eqs. (3.2) and (3.3) lead to the production of two muon neutrinos for every one electron neutrino (and zero tau neutrinos). This is contained in the neutrino flavour ratio of $\left(\nu_{e}: \nu_{\mu}, \nu_{\tau}\right) \simeq(1: 2: 0)$. While this is the expected flavour ratio at the source, over the long distances the flavours average out and the composition at the detector is expected to be near $\left(\nu_{e}: \nu_{\mu}: \nu_{\tau}\right) \simeq(1: 1: 1)$ [65]. This is shown here by calculating the oscillation probability and taking the long distance average.

In the three neutrino flavour case the mass eigenstates $\left(\nu_{1}, \nu_{2}, \nu_{3}\right)$ and flavour eigenstates $\left(\nu_{e}, \nu_{\mu}, \nu_{\tau}\right)$ are related by the PMNS matrix $U$ [65]:

$$
\left(\begin{array}{c}
\nu_{e} \\
\nu_{\mu} \\
\nu_{\tau}
\end{array}\right)=U\left(\begin{array}{c}
\nu_{1} \\
\nu_{2} \\
\nu_{3}
\end{array}\right)
$$

where each component of the PMNS matrix, $U_{i \alpha}$, shows the mixing of the flavours $\alpha$ into the three mass eigenstates $i$ where $\mathrm{U}$ is:

$$
U \equiv\left(\begin{array}{ccc}
U_{e 1} & U_{e 2} & U_{e 3} \\
U_{\mu 1} & U_{\mu 2} & U_{\mu 3} \\
U_{\tau 1} & U_{\tau 2} & U_{\tau 3}
\end{array}\right)
$$

In a vacuum the probability that a neutrino of flavour $\alpha$ will oscillate to flavour $\beta$ after a distance $L$ is [65]:

$$
\begin{aligned}
P\left(\nu_{\alpha} \rightarrow \nu_{\beta} ; L\right) & =\sum_{j=1}\left|U_{\beta j}\right|^{2}\left|U_{\alpha j}\right|^{2}+2 \sum_{i>j} \mathcal{R} e\left(U_{\beta j}^{*} U_{\alpha j} U_{\beta i} U_{\alpha i}^{*}\right) \cos \left(\frac{\Delta m_{i j}^{2} L}{2 E}\right) \\
& +2 \sum_{i>j} \mathcal{I} m\left(U_{\beta j}^{*} U_{\alpha j} U_{\beta i} U_{\alpha i}^{*}\right) \cos \left(\frac{\Delta m_{i j}^{2} L}{2 E}\right)
\end{aligned}
$$




\subsection{NEUTRINO PROPAGATION}

where $\Delta m_{i j}$ is the difference between square masses of the mass eigenstates $j$ and $k$, $E$ is the neutrino energy, and $L$ is the distance the neutrino travels.

Taking the limit of Eq. (3.6) as $L \rightarrow \infty$ and averaging over rapid oscillations this becomes:

$$
P\left(\nu_{\alpha} \rightarrow \nu_{\beta} ; L \rightarrow \infty\right)=\sum_{i=1}^{3}\left|U_{\beta i}\right|^{2}\left|U_{\alpha i}\right|^{2}
$$

The oscillation probability between each flavour can then be expanded as a matrix:

$$
P \equiv\left(\begin{array}{ccc}
P_{e e} & P_{e \mu} & P_{e \tau} \\
P_{e \mu} & P_{\mu \mu} & P_{\mu \tau} \\
P_{e \tau} & P_{\mu \tau} & P_{\tau \tau}
\end{array}\right) \equiv\left(\begin{array}{ccc}
\left|U_{e 1}\right|^{2} & \left|U_{e 2}\right|^{2} & \left|U_{e 3}\right|^{2} \\
\left|U_{\mu 1}\right|^{2} & \left|U_{\mu 2}\right|^{2} & \left|U_{\mu 3}\right|^{2} \\
\left|U_{\tau 1}\right|^{2} & \left|U_{\tau 2}\right|^{2} & \left|U_{\tau 3}\right|^{2}
\end{array}\right)=\left(\begin{array}{ccc}
0.55 & 0.25 & 0.2 \\
0.25 & 0.37 & 0.38 \\
0.2 & 0.38 & 0.42
\end{array}\right)
$$

where the PMNS values were used from [65]. Now, the extragalactic neutrino flux in the far distance can be expressed as a product of $P$ and the intrinsic flux $F^{0}\left(\nu_{\alpha}\right)(\alpha=$ $e, \mu, \tau)$ :

$$
\left(\begin{array}{c}
F\left(\nu_{e}\right) \\
F\left(\nu_{\mu}\right) \\
F\left(\nu_{\tau}\right)
\end{array}\right)=P\left(\begin{array}{c}
F^{0}\left(\nu_{e}\right) \\
F^{0}\left(\nu_{\mu}\right) \\
F^{0}\left(\nu_{\tau}\right)
\end{array}\right)
$$

With an initial flavour ratio assumed to be $\left(\nu_{e}: \nu_{\mu}: \nu_{\tau}\right)=(1: 2: 0)$ from pion 


\subsection{NEUTRINO PROPAGATION}

decay, the long distance flavour ratio can be found:

$$
\begin{aligned}
\left(\begin{array}{c}
F\left(\nu_{e}\right) \\
F\left(\nu_{\mu}\right) \\
F\left(\nu_{\tau}\right)
\end{array}\right) & =\left(\begin{array}{ccc}
\left|U_{e 1}\right|^{2} & \left|U_{\mu 1}\right|^{2} & \left|U_{\tau 1}\right|^{2} \\
\left|U_{e 2}\right|^{2} & \left|U_{\mu 2}\right|^{2} & \left|U_{\tau 2}\right|^{2} \\
\left|U_{e 3}\right|^{2} & \left|U_{\mu 3}\right|^{2} & \left|U_{\tau 3}\right|^{2}
\end{array}\right)\left(\begin{array}{c}
1 \\
2 \\
0
\end{array}\right) \\
& \simeq\left(\begin{array}{c}
1 \\
1 \\
1
\end{array}\right)
\end{aligned}
$$

Therefore, the ratio of the cosmic high-energy neutrino fluxes over extragalactic distances is about $\left(\nu_{e}: \nu_{\mu}: \nu_{\tau}\right) \simeq(1: 1: 1)$. A flavour ratio consistent with this has been observed at IceCube as shown in Fig. 3.2. In this thesis a flavour ratio of $\left(\nu_{e}: \nu_{\mu}: \nu_{\tau}\right)=(1: 1: 1)$ at the detector is assumed. This is reasonable given the low statistics possible at present detectors and uncertainty about the oscillation parameters at the source $[8,66,67]$.

\subsubsection{Neutrino Attenuation in Matter}

\section{Neutrino attenuation in the Earth}

In determining the attenuation of an extra-galactic neutrino flux as observed on Earth, the interaction with visible matter should be considered. The propagation length of neutrinos can be characterized by its mean free path, $\lambda$, which describes the distance a particle travels before scattering [68]:

$$
\lambda_{\text {matter }}=\frac{1}{n \sigma} .
$$




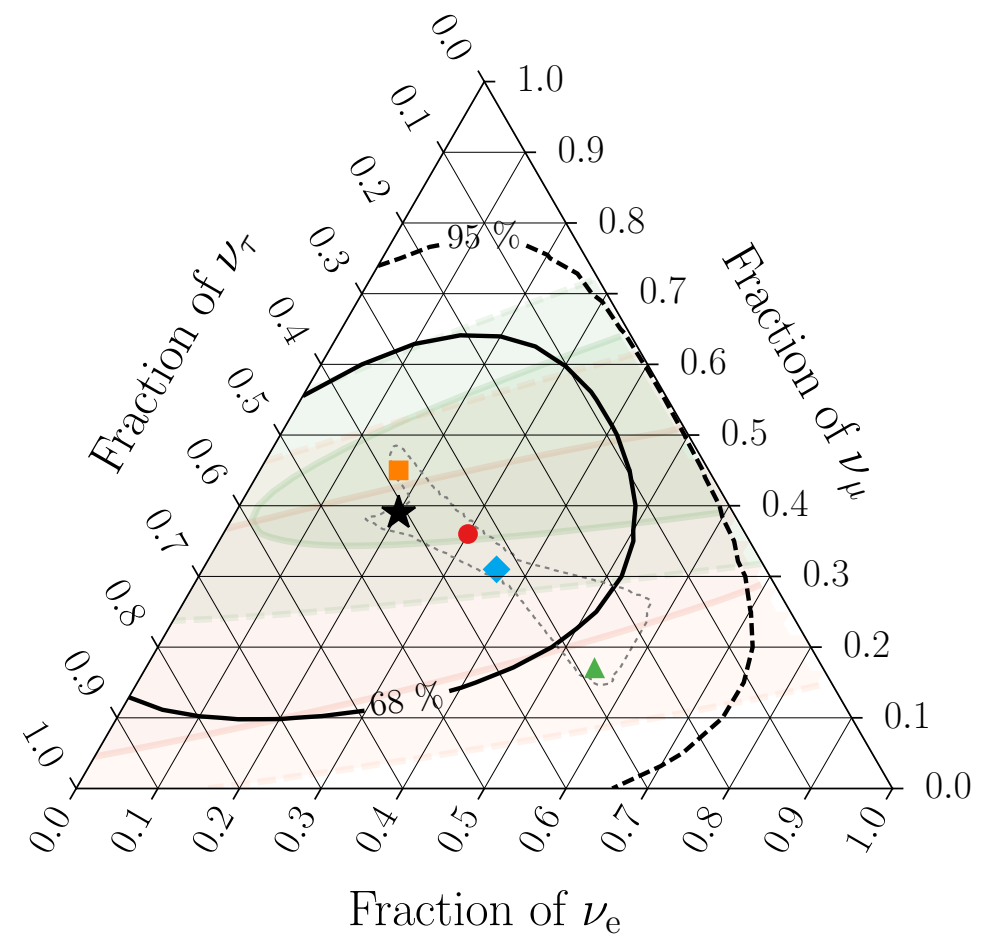
$\nu_{e}: \nu_{\mu}: \nu_{\tau}$ at source $\rightarrow$ on Earth:
$0: 1: 0 \rightarrow 0.17: 0.45: 0.37$
- $1: 2: 0 \rightarrow 0.30: 0.36: 0.34$
$\Delta \quad 1: 0: 0 \rightarrow 0.55: 0.17: 0.28$
- $1: 1: 0 \rightarrow 0.36: 0.31: 0.33$

Figure 3.2: Triangle plot of flavour ratio observed in IceCube's 7.5 year high energy starting event sample. While the flavour ratio is not strongly constrained it is consistent with a source ratio of (1:2:0) which would indicate neutrino production from pion decay (red marker). The other coloured markers show different flavour ratios at neutrino production, that are all allowed.The best fit point is shown with the black star, and $1 \sigma$ and $2 \sigma$ contours are shown with black lines. Figure from Ref: [8]. 


\subsection{NEUTRINO PROPAGATION}

where $n$ is the number density of intervening particles and $\sigma$ is the scattering cross section.

In the case of neutrinos scattering in the Earth, neutrino-nucleon deep elastic scattering is the dominant mechanism, where the number density $\sim N_{A} / \mathrm{cm}^{3}$, and the cross section for neutrino weak interactions in the lab frame is roughly $\sigma_{\text {lab }} \sim$ $G_{F}(E M) \sim 10^{-38} \mathrm{~cm}^{2} \frac{E M}{\mathrm{GeV}^{2}}[68,69]$. Here $E$ is the energy of the neutrino, $M$ is the mass of the nucleon, $N_{A}$ is Avogadro's number, and $G_{F}$ is the Fermi constant. For nucleons with masses on the order $1 \mathrm{GeV}$ the mean free path is then:

$$
\lambda_{\text {matter }}=\frac{1}{n \sigma} \sim \frac{10^{14} \mathrm{~cm}}{\left(E_{\nu} / \mathrm{GeV}\right)} .
$$

With the diameter of the Earth as $10^{9} \mathrm{~cm}$, this means that the Earth starts to become opaque to neutrinos above $100 \mathrm{TeV}$. This is confirmed with numerical simulation [70]. While neutrino detectors often use the Earth as a shield against the main background of muons, the flux of high energy astrophysical neutrinos will also be attenuated to a degree after passing through the Earth. This motivates the use of neutrino detectors in the Southern Hemisphere that use advanced veto techniques to observe high energy neutrinos in the direction of the Galactic Centre.

\section{Neutrino Attenuation in the Intergalactic Medium}

An estimate for the attenuation of the astrophysical neutrino flux after scattering with baryonic matter in the intergalactic medium can be found to confirm that normal matter interactions are negligible. Assuming the baryonic matter in the galaxy can 


\subsection{NEUTRINO PROPAGATION}

be separated into a disk and bulge, the bulge density is described as [71]:

$$
\rho_{b}=\frac{\rho_{b, 0}}{\left(1+r^{\prime} / r_{0}\right)^{\alpha}} \exp \left[-\left(r^{\prime} / r_{c u t}\right)^{2}\right],
$$

with $r^{\prime}=\sqrt{R^{2}+(z / q)^{2}}$, which was found to have best fit values of $\alpha=0.18, r_{0}=$ $0.075 \mathrm{kpc}, r_{\text {cut }}=2.1 \mathrm{kpc}, q=0.5$ and $\rho_{b, 0}=9.93 \times 10^{10} M_{\odot}$. The disk density is described as [71]:

$$
\rho_{d}(R, z)=\frac{\Sigma_{d, 0}}{2 z_{d}} \exp \left(-\frac{|z|}{z_{d}}-\frac{R}{R_{d}}\right),
$$

which was found to have best fit value of scale height $z_{d} \approx 0.6 \mathrm{kpc}$, scale radius $R_{d} \approx 3 \mathrm{kpc}$ and central density $\Sigma_{d, 0} \approx 60 M_{\odot} \mathrm{pc}^{-2}$.

With these a column density can be estimated as:

$$
\tau(\vec{x})=\int_{\text {l.o.s }} n(\vec{x}) d x,
$$

from which the mean free path is $\lambda=(\tau \sigma)^{-1}$. For the aforementioned disk and bulge distributions, a path with an angle near the Galactic Centre leads to a column density $\tau \sim 1 \mathrm{~g} / \mathrm{cm}^{3}$. For a cross section $\sigma_{\text {lab }} \approx G_{F} E M \approx 10^{-38} \mathrm{~cm}^{2} \frac{E M}{\mathrm{GeV}^{2}}$ the mean free path through galactic matter is on the order of

$$
\lambda_{\text {baryon }} \approx \frac{10^{38} \mathrm{~cm}}{(E / \mathrm{GeV})} .
$$

Considering that the Milky Way is on the order of $60 \mathrm{kpc} \sim 1 \times 10^{23} \mathrm{~cm}$ in diameter and that neutrinos observed by IceCube are $\mathrm{GeV}-\mathrm{PeV}$, scattering effects between baryonic matter and neutrinos can be neglected. 


\subsection{DETECTING NEUTRINOS}

\subsection{Detecting Neutrinos}

Due to the weakly interacting nature of neutrinos, a large detector volume is required in order to detect a statistically significant number of neutrino events. This requirement has led to the construction of numerous water Cherenkov detectors around the world. These detect the optical signals that come from charged particles traveling faster than the local speed of light in a medium. While these detectors do not directly detect neutrinos, the optical signal (Cherenkov radiation) from secondary charged particles can be used to reconstruct the energy and direction of the associated neutrino. The arrival time of photons is used to determine the particles trajectory and the number of photons is used as a proxy for the deposited energy [10].

These secondary particles that provide insight into their parent neutrino are the result of deep inelastic scattering with the quarks in a nucleus in the medium. In these processes the nucleus breaks apart and leads to hadronic shower that can be observed using Cherenkov radiation. This weak interaction between the neutrino and up or down quark can either be a charged current (CC) when mediated by a W boson, or a neutral current (NC) when mediated by a Z boson [10]. The Feynman diagrams for these interactions is shown in Fig. 3.3.

While neutral current interactions leave the neutrino intact, charged current interactions produce a charged lepton associated with the initial neutrino flavour. At these energies $80 \%$ of the neutrino energy is transferred to its charged lepton partner [72].

\subsubsection{IceCube}

IceCube is a cubic kilometer ice Cherenkov detector located at the geographic South Pole just outside the Amundsen-Scott South Pole Station. It is composed of 5160 


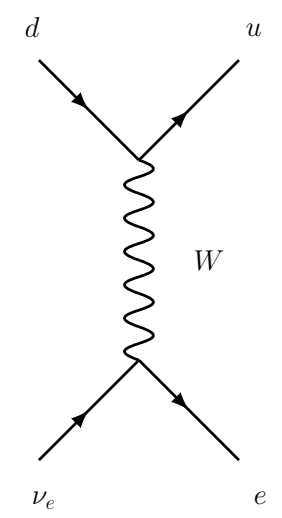

(a) $\mathrm{CC} \nu_{e}$

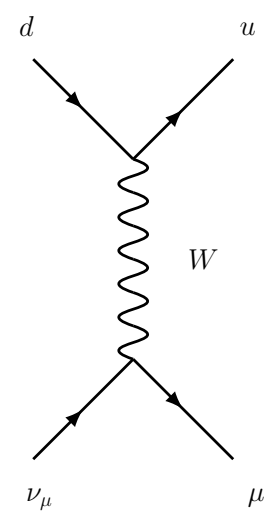

(b) $\mathrm{CC} \nu_{\mu}$

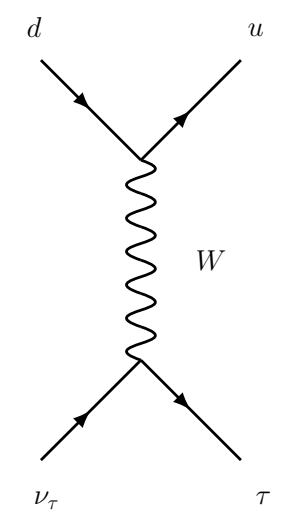

(c) $\mathrm{CC} \nu_{\tau}$

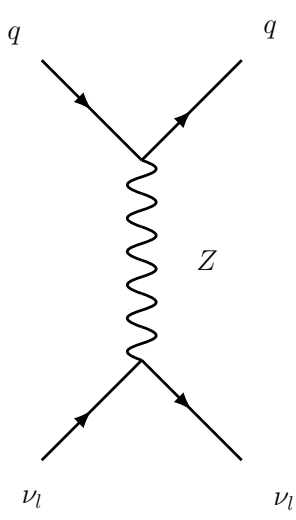

(d) $\mathrm{NC}$

Figure 3.3: Charged and Neutral current interactions Feynman diagrams representing the interactions that IceCube can detect.

digital optical modules (DOMs) that detect Cherenkov light from charged particles which are produced after high energy neutrinos interact with the ultraclear ice [10].

The large volume of the detector allows for a sufficient number of neutrino events to be observed. A graphic of IceCube's configuration is shown in Fig. 3.4. 


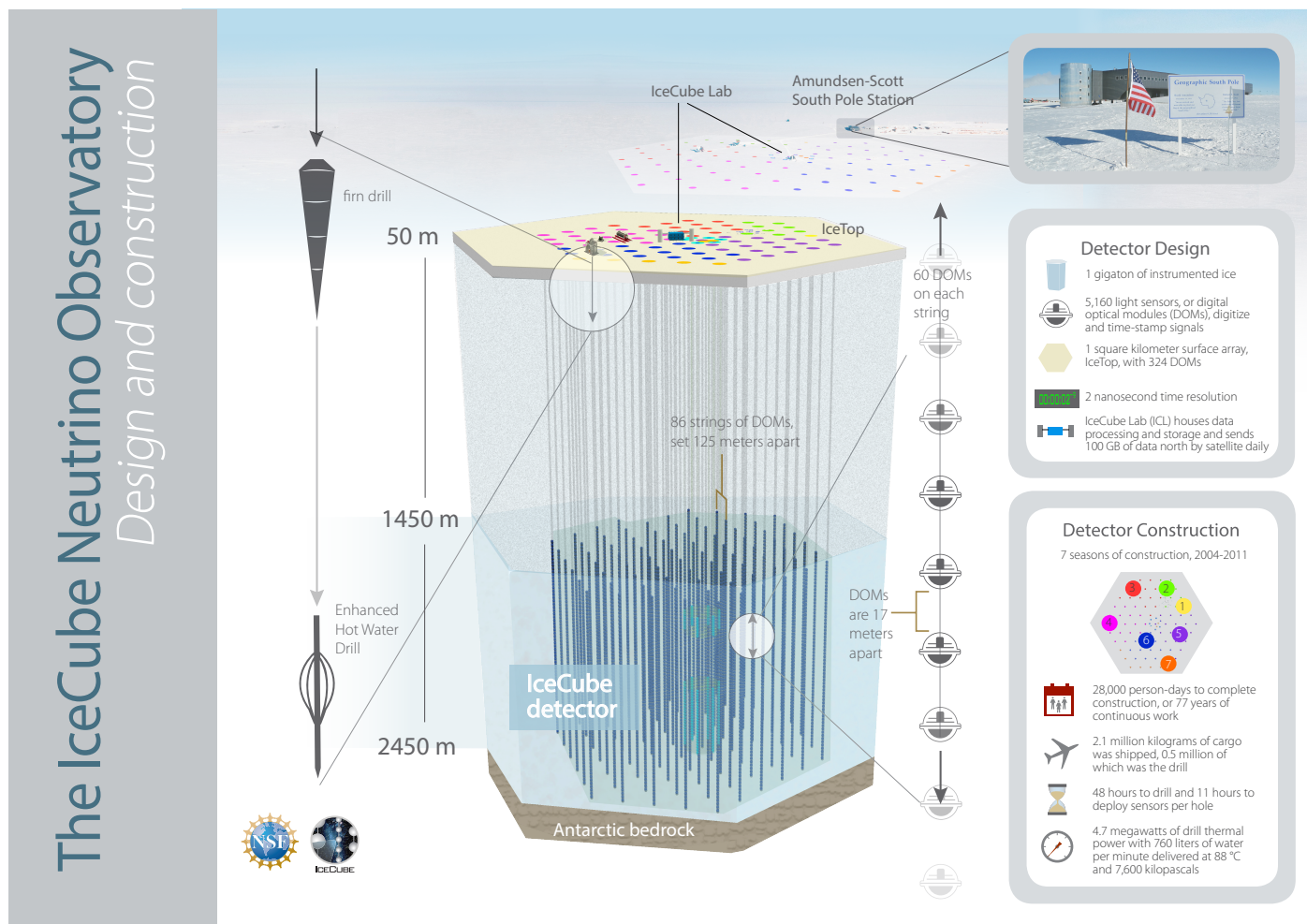

Figure 3.4: Graphical layout of IceCube [9]. The detector is composed of 86 vertical strings suspended in the ice across a hexagonal grid with $125 \mathrm{~m}$ spacing. Each string contains $60 \mathrm{DOMs}$ that are each separated by $17 \mathrm{~m}$. The DOM are buried from $1450 \mathrm{~m}$ to $2450 \mathrm{~m}$ below the ice's surface. IceCube is composed of a surface array, IceTop, and a denser inner subdetector, DeepCore, that significantly enhances the capabilities of the observatory, making it a multipurpose facility. DeepCore lowers the neutrino energy threshold to $10 \mathrm{GeV}[10]$.

\subsubsection{Event Morphologies}

Events at IceCube are generally described by the optical signature that is left in the detector and separated into three morphologies: track, cascade, and double bang. Characteristic events for each event morphology are shown in Fig. 3.5. 


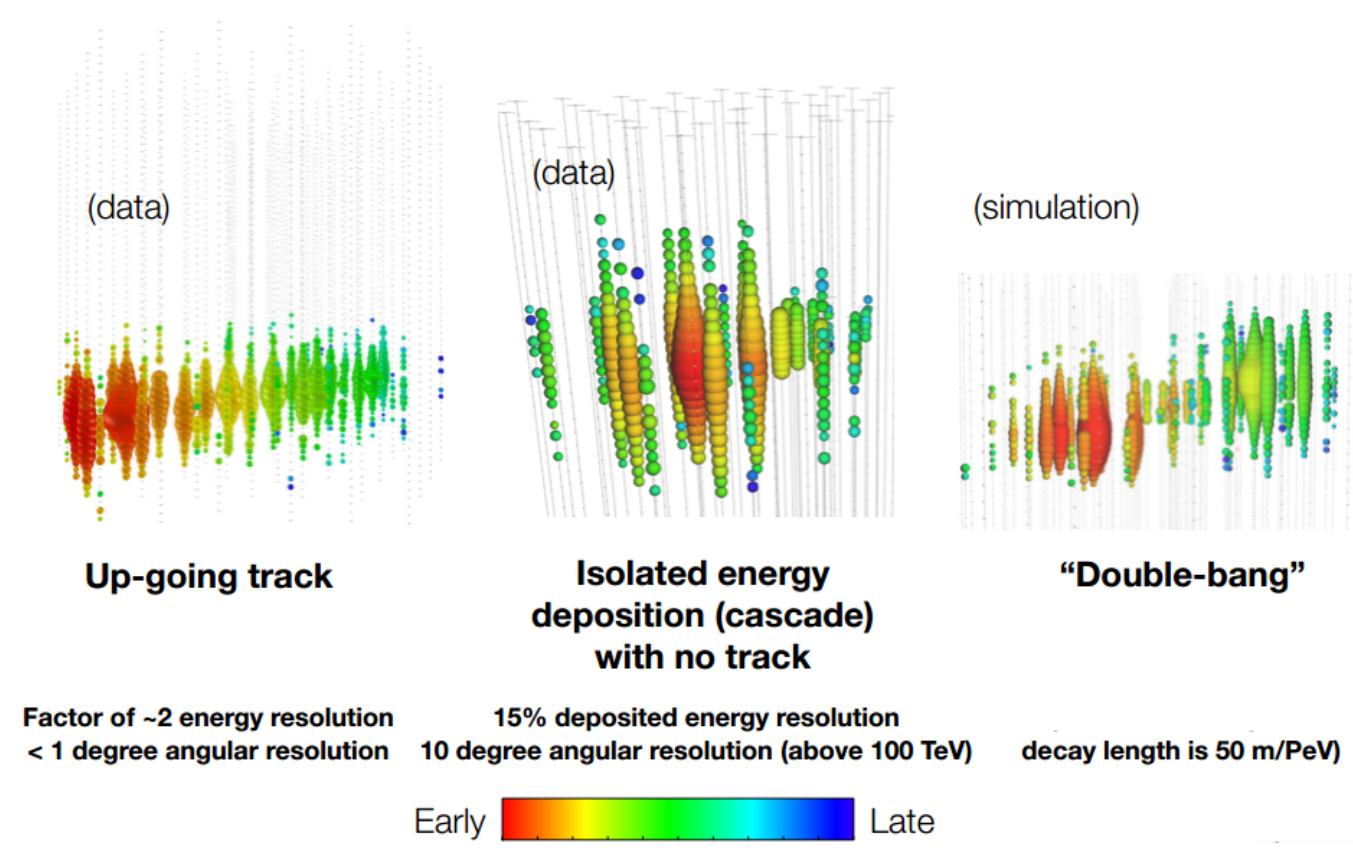

Figure 3.5: Different event morphologies observed at IceCube from tracks, cascades or 'double bangs'. Figure from [11].

\section{Cascades}

Cascade events come from the hadronic particle showers that follow the neutrino scattering of the nucleus in the ice. These are highly localized signals where the repeated scattering and production of secondary particles leads to a distinct spherical signal in the detector. Cascade type events include all flavours of neutrinos from $\mathrm{NC}$ and electron and tau neutrinos from CC interactions. They have about a $15 \%$ energy resolution and $10^{\circ}$ angular resolution above $100 \mathrm{TeV}[2]$. 


\subsection{DETECTING NEUTRINOS}

\section{Track}

Track events are caused by muons that pass through the detector leaving a long line of Cherenkov light. These tracks typically originate outside of the detector from CC interactions and form a kilometer long track through the detector. Upgoing track events that have traveled through the Earth have $\leq 0.3^{\circ}$ angular resolution at 100 $\mathrm{TeV}$, however, have a weak energy resolution that is $\sim 2 E_{\nu}$. Certain veto techniques, such as the starting events method used in the HESE 7.5 analysis (and described in 3.5.3) can improve this energy resolution to $30 \%$ for energies above $10^{4} \mathrm{GeV}$ [13].

\section{Double cascades}

Since the tau neutrino has a very short lifetime, a large boost is required to observe a double cascade. As such these events are rare with only two detected in 7.5 years of data. These events occur when a tau neutrino interacts twice within the detector volume via charged current interactions. In these type of events, the first cascade is from the hadronic interaction of a tau neutrino to produce a tau and the second cascade comes from the tau decaying to a hadronic or electromagnetic cascade [8].

\subsubsection{Distinguishing Astrophysical Events}

There exists a large background of muons from cosmic ray interactions in the atmosphere for which various techniques are used to isolate an astrophysical neutrino signal. At IceCube there are three ways to differentiate the astrophysical neutrino signal from the background of atmospheric neutrinos and atmospheric muons: 1) observing tracks from upgoing muons and 2) using a veto region 3) look at very high energies where the astrophysical flux dominates [13]. 


\subsection{DETECTING NEUTRINOS}

\section{Upgoing Tracks}

In the tracks method the Earth is used as a shield against the muon background. Since neutrinos travel through the Earth unimpeded, this means that any track events that are upgoing (having passed though the Earth) came from neutrino events. This is effective for eliminating the atmospheric muon flux above $100 \mathrm{TeV}$, but is only sensitive to the northern hemisphere and muon neutrinos [13].

\section{Starting Events}

The starting events selection technique involves separating the detector volume into an outer veto region and an inner detector region. In this case any track events that are recorded in an outer veto region are removed, eliminating muons that come from outside the detector. In this case only events that have a neutrino-nucleon interaction vertex located inside of the detector are accepted. This is shown in Figs. 3.6. Here a muon track that passes through the veto region is excluded, while a neutrino that travels through the veto region and interacts within the inner detector region is allowed. This technique allows events from both the northern and southern skies to be recorded [2].

Similarly, the atmospheric and astrophysical neutrino events can be differentiated by searching for coincident muon tracks and neutrino cascades. Since the air showers that produce atmospheric neutrinos also produce atmospheric muons, coincident track and cascade events are likely to be from atmospheric neutrinos. This requires that the muon is sufficiently energetic and at a small zenith angle. This also allows one to include down-going astrophysical neutrino events and a full view of the neutrino sky. This is shown in Fig. 3.7. 


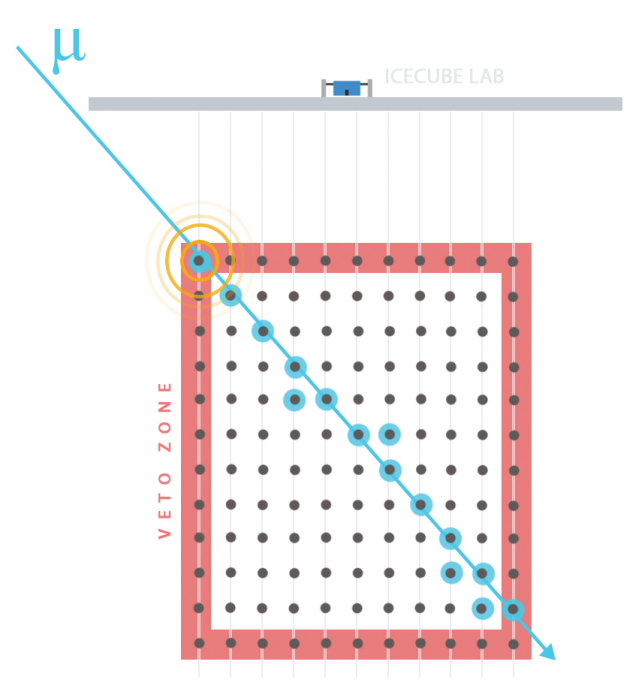

(a) Charged current $\nu_{e}$

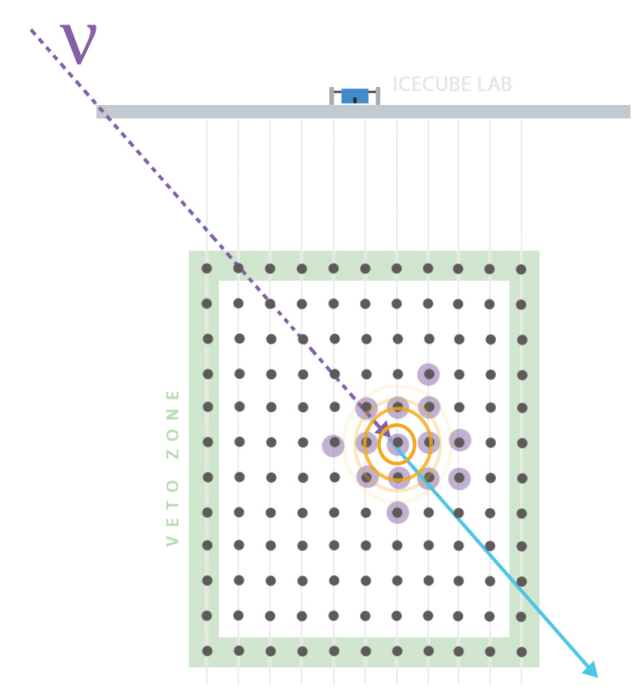

(b) Charged current $\nu_{\mu}$

Figure 3.6: The veto region can be used to differentiate neutrinos from atmospheric muons. a) A muon that enters the detector is vetoed while b) a neutrino can pass through the veto region, interact within the detector volume and be recorded as a neutrino event. Fig. from [9].

\section{Observing High Energies}

As the atmospheric neutrino flux dominates below energies of $10^{2} \mathrm{TeV}$, a common veto technique involves removing events below $10^{2} \mathrm{TeV}$ from the data. For energies below this cutoff the previously described veto techniques become important [2]. The astrophysical and atmospheric fluxes are shown in 3.8, where the dominance of the atmospheric flux at low energies can be observed. At IceCube a unique veto technique that is described by the previous veto methods is used to form datasets that have a high number of astrophysical events. These include both the high energy starting events (HESE) and medium energy starting events (MESE) datasets that extend from energies $30 \mathrm{TeV}-10 \mathrm{PeV}$ and $1 \mathrm{TeV}-10 \mathrm{PeV}$ respectively [2,13]. 


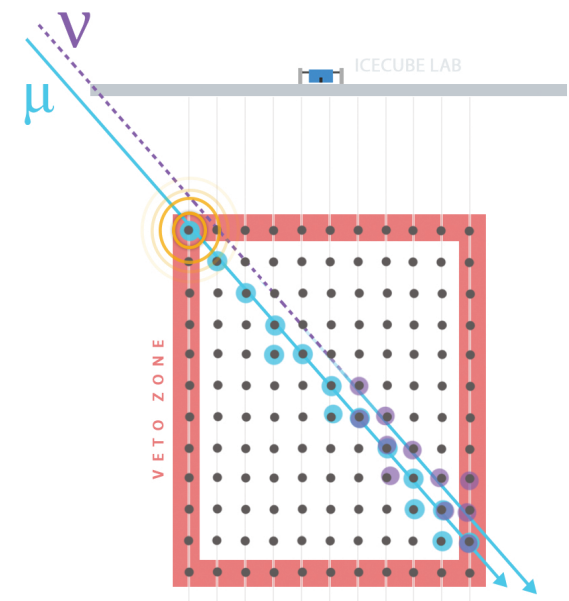

Figure 3.7: The veto region is used to remove an atmospheric neutrino that is coincident with a muon from the signal [9].

\subsection{Modelling Neutrinos Observed at IceCube}

\subsubsection{Astrophysical Sources}

The astrophysical component of the neutrinos observed at IceCube is affected by DM-neutrino scattering and is the source of the signal for this analysis. There are a variety of flux models to describe the astrophysical neutrinos as shown in Fig. 3.9. As discussed in Sec. 3.2, while there is evidence supporting particular point sources of neutrinos such as active galactic nuclei, the exact source of extragalactic neutrinos remains uncertain. As such, this analysis takes an agnostic approach to the astrophysical neutrino source and assumes an isotropic distribution that is consistent with theory and observation. This distribution implies a diffuse extra-galactic origin from a large number of sources. It is also assumed that the energy spectrum of the neutrino 


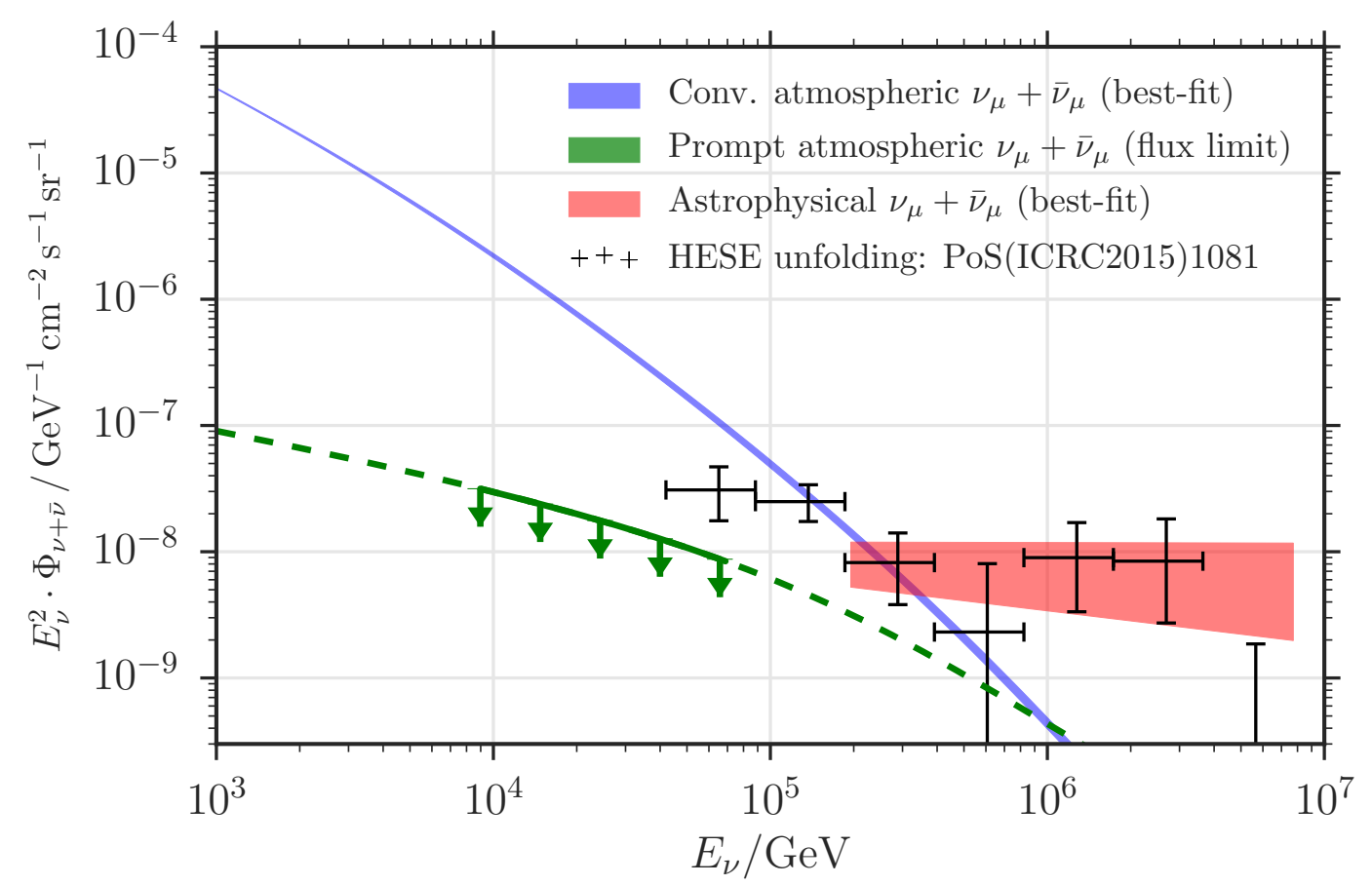

Figure 3.8: The atmospheric neutrino flux at IceCube is dominated by conventional rather than prompt neutrinos. Figure from Ref: [12].

flux $\frac{d \Phi}{d E_{\nu}}$ follows a power law:

$$
\frac{d \Phi_{\text {astro }}}{d E_{\nu}}=\phi_{\text {astro }}\left(\frac{E_{\nu}}{100 \mathrm{TeV}}\right)^{-\gamma} \times 10^{-18} \mathrm{GeV}^{-1} \mathrm{~cm}^{-2} \mathrm{~s}^{-1} \mathrm{sr}^{-1}
$$

for neutrino energy $E_{\nu}$, a spectral index of $\gamma$ and flux normalization constant $\phi_{\text {astro }}$ at $100 \mathrm{TeV}$. This type of power law is motivated by Fermi acceleration mechanisms where the normalization constant of the high energy astrophysical neutrino flux relates to interaction conditions at the sources. The best fits for $\phi_{\text {astro }}$ and $\gamma$ are shown in Fig. 3.10, where it can be seen that these are constrained to about $\gamma \approx 2-3$ and $\phi_{\text {astro }} \approx 2-10$. While this thesis considers an isotropic extragalactic source of astrophysical neutrinos, it is possible that a portion astrophysical neutrinos come 


\subsection{MODELLING NEUTRINOS OBSERVED AT ICECUBE}

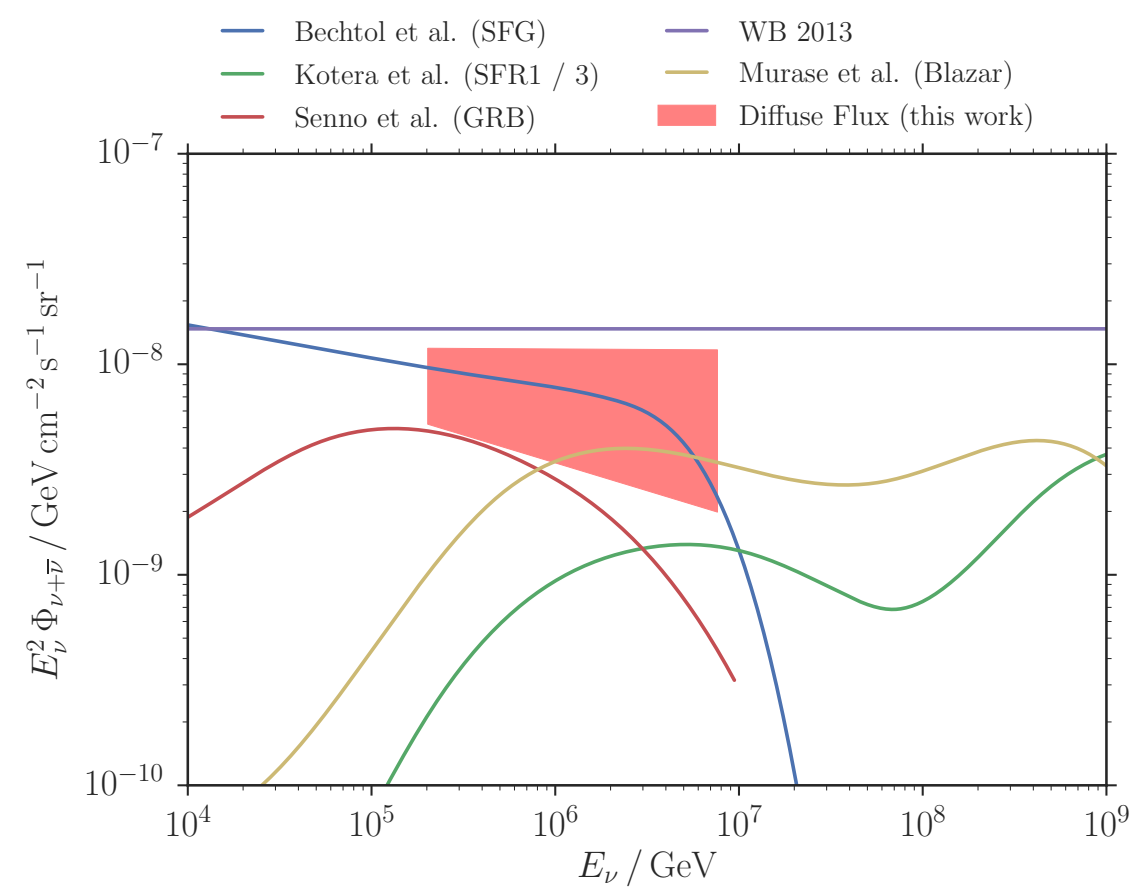

Figure 3.9: There are many different models to describe the astrophysical neutrino flux. Figure from Ref: [12].

possible galactic source. This contribution has been constrained to a $10 \%$ contribution at $90 \%$ confidence, with a best fit for the galactic contribution being $\sim 1 \%$ of the astrophysical neutrino flux [73].

\subsubsection{Atmospheric Neutrinos}

Atmospheric neutrinos constitute a significant background at IceCube for energies up to $100 \mathrm{TeV}$. They are produced when cosmic rays interact with the Earth's atmosphere to produce mesons. The common air showers that are considered are shown in Fig. 3.11. There are two classes of atmospheric neutrinos depending on their decay chain: conventional and prompt [74]. Conventional neutrinos come from the decay of cosmic protons into charged pions or kaons $\left(p \rightarrow \pi^{+}\right)$, while the prompt flux comes 


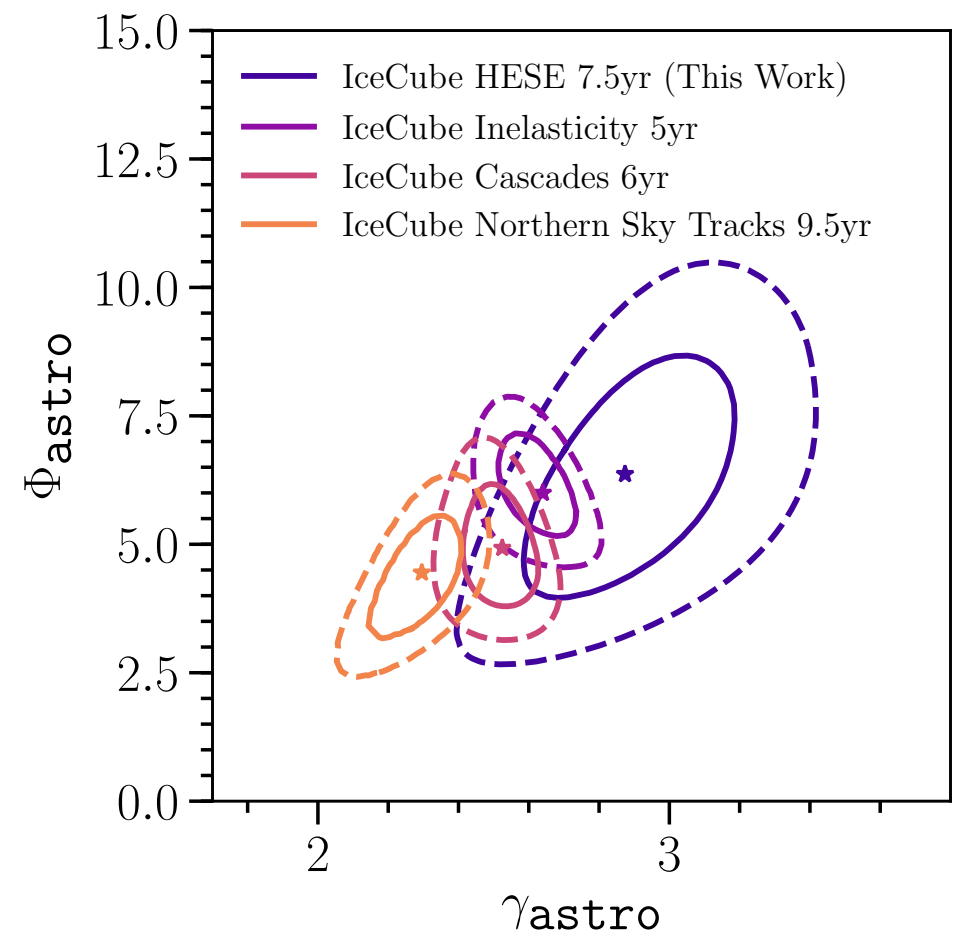

Figure 3.10: Best fits for the spectral index and flux normalization constant [13].

from the decay of heavier mesons (containing a charm quark). The pions then decay to muons of Eq. (3.2).

The pions and kaons in the conventional flux have enough time to decay in the atmosphere and lose energy. As such, the conventional flux is expected to be softer than its parent cosmic ray spectrum and behaves as $\sim E^{-3.7}$. At high energies this is dominated by muon neutrinos and is largest at the horizon after having passed through the greatest atmospheric column density [75].

The prompt flux is from the decay of heavier mesons like D mesons with $\nu_{e}$ and $\nu_{\mu}$ in a 1:1 ratio. This flux is expected to follow a $\sim E^{-2.7}$ power law that is isotropic. Neutrinos from the prompt channel have yet to be measured and in this thesis the 


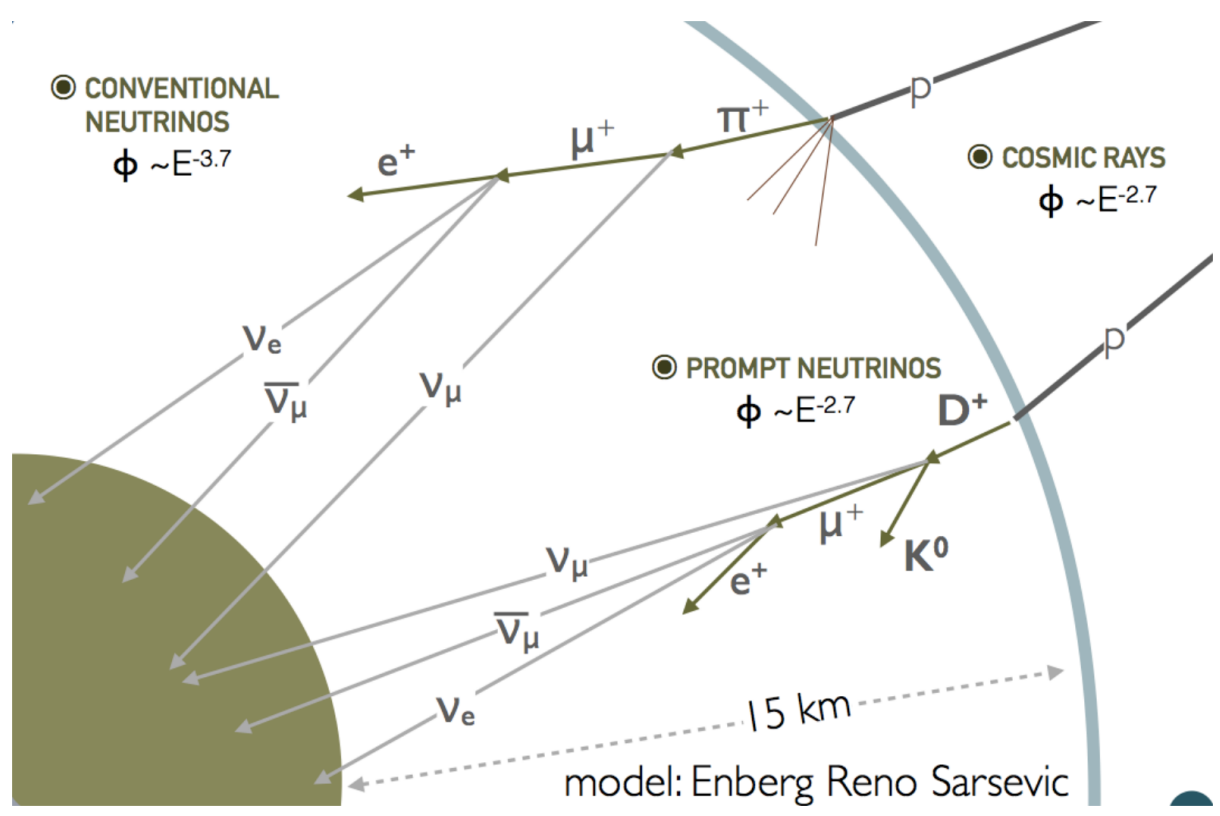

Figure 3.11: The cosmic ray air shower that leads to the production of the background atmospheric neutrinos observed at IceCube. The interactions shown are not complete and does not show the associated electromagnetic component and muon shower. Figure from Ref: [14].

prompt flux is assumed to be negligible [75].

Conventional neutrinos dominate the atmospheric flux except at high energies (hundreds of $\mathrm{TeV}$ ). However, at these high energies the astrophysical neutrinos begin to dominate as shown in Fig. 3.8. The atmospheric neutrino flux model used in this thesis is described as [13]:

$$
\frac{d \Phi_{a t m}}{d E_{\nu}}=\phi_{\text {prompt }}\left(\phi_{\pi}+R_{K} \phi_{K}\right)\left(\frac{E_{\nu}}{E_{0}}\right)^{\Delta \gamma}+\phi_{\text {conv }}\left(\frac{E_{\nu}}{E_{0}}\right)^{\Delta \gamma}
$$

where $\phi_{\text {prompt }}$ and $\phi_{\text {conv }}$ are the normalizations to the prompt and conventional fluxes at neutrino energy, $E_{\nu}, \phi_{\pi}$ and $\phi_{K}$ are the normalizations for the kaon and pions, $R_{K}$ modifies the relative kaon and pion contributions, and $\Delta \gamma$ allows for hardening or softening of these components. $E_{0}=2020 \mathrm{GeV}$ is a pivot energy for the adjusted 


\subsection{MODELLING NEUTRINOS OBSERVED AT ICECUBE}

spectral index. In this thesis the atmospheric neutrino flux is modelled using nuFlux with a Honda-Gaisser model that only includes the conventional component [76]. As such Eq. (3.18) reduces to:

$$
\Phi_{a t m}=\phi_{a t m} w_{H G}\left(\frac{E_{\nu}}{E_{0}}\right)^{\Delta \gamma},
$$

where $\phi_{a t m}$ is the overall normalization of the atmospheric flux, $w_{H G}$ is the HondaGaisser weighting from nuFlux, and $\Delta \gamma$ is a spectral hardening/softening factor, which has a pivot point at $E_{0}=2020 \mathrm{GeV}$.

\subsubsection{Atmospheric Muons}

The signals detected at IceCube are dominated by cosmic ray muons. For every neutrino detected in IceCube, $10^{6}$ muons interact in the detector. These are produced in air showers when cosmic rays interact in the atmosphere. In this thesis these are computed with the muongun software [77].

For this analysis it is important to accurately model the neutrino background, so that a signal from possible DM-neutrino scattering can be detected. This signal from DM-neutrino scattering is described in the next chapter. 


\section{Chapter 4}

\section{Neutrino-Dark Matter Interactions}

The Standard Model (SM) of particle physics is the most widely accepted theory for describing all known elementary particles. However, numerous experiments have demonstrated the need for new theories to explain Beyond the Standard Model (BSM) physics. Examples of BSM phenomena include the non-zero mass of neutrinos and the (possible particle) nature of dark matter (DM). Of particular interest for this thesis is the mechanism of DM-neutrino interaction, which is a possible means to connect DM with the Standard Model.

\subsection{Dark Matter Detection}

DM researchers colloquially segment DM particle search techniques into three main categories: 'make it', 'break it' or 'shake it'. These correspond to the detection of DM via: production at colliders, indirect detection and direct detection. These strategies all involve detecting possible interactions between SM and DM particles. While these searches are each optimal for different DM models, they provide complementary strategies that are all important in the detection of DM. The schematic diagrams of these detection methods are shown in Fig. 4.1. 

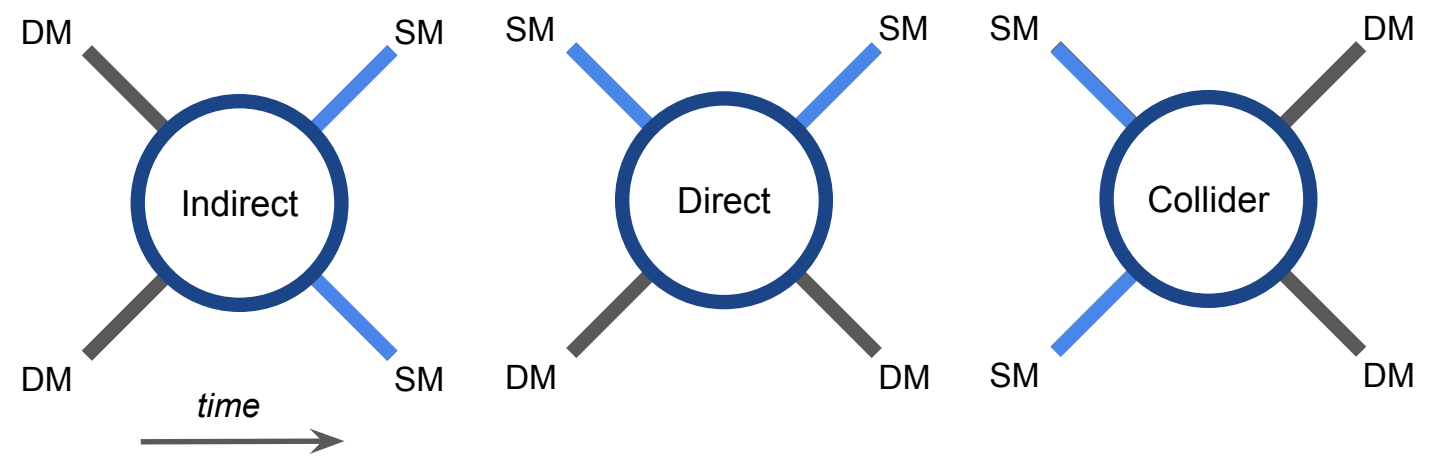

Figure 4.1: Schematic diagram for the different methods of detecting DM. Indirect detection involve the production of Standard Model (SM) particles from dark matter (DM). Direct detection involves the scattering of DM with SM. Collider techniques involve producing DM from SM.

\subsubsection{Production at Colliders}

Particle colliders such as the Large Hadron Collider have been instrumental in many of the particle physics discoveries of the last century, especially the recent discovery of the Higgs boson [78]. They can also be used in the hunt for for DM. This would involve accelerating Standard Model particles (such as protons) to collide, then searching the detectors for missing energy that could be DM [79]. Searches are restricted by the energy range that colliders can achieve $(<13 \mathrm{TeV}$ centre-of-mass) and timescales with which particles traverse the detector. Collider searches have the advantage of allowing for precision measurements in controlled experiments, enabling high levels of statistics [80].

\subsubsection{Direct Detection}

Direct detection involves detecting recoils from interactions of DM particles with target nuclei or electrons in detectors. This elastic scattering will lead to a release 
of energy through heat, ionization or scintillation that can be observed. These are typically performed underground to shield from cosmic ray backgrounds. The greatest challenges with this method are the size requirements to achieve a sufficient signal and distinguishing a signal from a background [81].

\subsubsection{Indirect Detection}

Indirect detection involves using astronomical observations of Standard Model particles that come from DM decay or annihilation [82]. These interactions can be observed in the fluxes of SM products, in particular gamma rays, charged particles and neutrinos [83]. Neutral particle (neutrino and gamma) searches are typically done by searching for signals from sources with higher DM concentrations [84], while cosmic ray antiparticles can also provide insight into dark matter [85]. Sources that would be expected to provide a strong DM signal include the galactic DM halo [86], the Galactic Centre [87], dwarf spheroidal galaxies [88], the CMB as well as signals from the Sun [89] and the Earth [90].

\section{Decay}

DM decay into standard model particles is a means of indirect detection to probe the properties of DM [91]. In the case of DM decaying into neutrinos this could lead to an excess in the neutrino flux in the direction of the Galactic Centre [92]. For galactic halo searches the neutrino flux from DM decay is given as:

$$
\frac{d \Phi_{\nu}}{d E_{\nu}}=\frac{1}{4 \pi} \frac{1}{m_{\chi} \tau_{\chi}} \frac{d N_{\nu}}{d E_{\nu}} \int_{0}^{\Delta \Omega} d \Omega \int_{\text {l.o.s. }} \rho_{\chi}(s, \phi, \theta) d s,
$$


where $m_{\chi}$ is the DM mass, $\frac{d N_{\nu}}{d E_{\nu}}$ is the DM neutrino spectrum per decay, $\Delta \Omega$ is the angular region of interest, $\tau_{\chi}$ is the DM lifetime, and $\rho_{\chi}$ is the DM density profile which is integrated over the line of sight (l.o.s).

\section{Annihilation}

DM annihilation into standard model particles is supported by the WIMP scenario in the case of a continued self-annihilation into SM particles. Here the WIMP miracle predicts a thermally averaged self annihilation rate of $\langle\sigma v\rangle=3 \times 10^{-26} \mathrm{~cm}^{3} \mathrm{~s}^{-1}$ [93]. Common annihilation products of DM that are used in analyses are $W^{ \pm}, Z, b \bar{b}$, $\mu, \tau$, and $\nu$. These can subsequently annihilate or decay to electrons/positrons, protons/anti-protons, photons, or neutrinos. In Galactic Centre searches, DM annihilation into neutrinos would lead to an excess of neutrinos in the direction of the Galactic Centre. The neutrino flux from DM annihilation is generically:

$$
\frac{d \Phi_{\nu}}{d E_{\nu}}=\frac{1}{4 \pi} \frac{\left\langle\sigma_{A} v\right\rangle}{2 m_{\chi}^{2}} \frac{d N_{\nu}}{d E_{\nu}} \int_{0}^{\Delta \Omega} d \Omega \int_{\text {l.o.s. }} \rho_{\chi}^{2}(s, \phi, \theta) d s
$$

This neutrino flux depends on the annihilation cross section $\left\langle\sigma_{A} v\right\rangle$, the DM mass $m_{\chi}$, the final state energy spectrum $\frac{d N_{\nu}}{d E_{\nu}}$, as well as the DM density distribution along the l.o.s, $\rho_{\chi}$. In the case of IceCube, a DM annihilation search has been performed for the Galactic Centre, where limits down to $7.44 \times 10^{-24} \mathrm{~cm}^{3} \mathrm{~s}^{-1}$ were found for a 200 GeV DM particle annihilating through the $\tau^{+} \tau^{-}$channel [87,93,94]. Better limits can also be achieved with gamma ray studies which have placed upper limits on the thermally averaged cross section at $\left\langle\sigma_{A} v\right\rangle \lesssim 10^{-26} \mathrm{~cm}^{3} \mathrm{~s}^{-1}$ for a $200 \mathrm{GeV}$ dark matter through annihilation to $\tau^{+} \tau^{-}[84]$. 


\subsection{SCATTERING}

\subsection{Scattering}

The mechanism that is explored in this thesis is the scattering of DM with standard model particles. Possible DM scattering interactions that have been previous explored involve quarks [95], electrons [16], photons [96,97] or neutrinos [1]. Searching for DM scattering with neutrinos has the benefit that neutrinos rarely interact with normal matter and can point directly back to their source. In this thesis, scattering between neutrinos and DM is considered with respect to the altered neutrino flux that would be observed on Earth.

\subsection{Dark Matter-Neutrino Scattering}

In addition to augmenting the information from electromagnetic astronomy, the observation of high-energy neutrinos also provides a novel means through which to probe the properties of DM [22]. While DM interactions with neutrinos are one of the least constrained possible interactions, bounds have been placed from the CMB and largescale structure for scattering at low energies [15,98-106]. However, for high neutrino energies, the DM-neutrino cross section is weakly constrained due to a lack of observational data [107]. DM-neutrino models are especially attractive for light DM models, where annihilation into heavier products is forbidden and appears naturally in models like the sterile neutrino. Searching for interactions at the high energies observed at IceCube is important as the scattering cross section scales with energy.

While this thesis considers how the neutrino-DM scattering would affect the neutrino spectrum as observed at IceCube, other research has considered the effect of boosting DM via such scattering interactions [108,109]. This type of signal would require different interaction mechanisms to be observed (electron-DM scattering). 


\subsection{DARK MATTER-NEUTRINO SCATTERING}

DM-neutrino scattering has also been considered in terms of the time delay between neutrino and photon signals as a result of DM neutrino scattering [110].

Similar work on the spectral variation was previously considered for 37 neutrino events detected at IceCube over 988 days with energies ranging from 30-2000 TeV. An upper limit of $\sigma \leq 10^{-17}\left(m_{\chi} / \mathrm{GeV}\right)\left(E_{\nu} / \mathrm{PeV}\right)^{2} \mathrm{~cm}^{2}$ was found from neutrinos scattering in with extragalactic DM [107]. They also considered extragalactic neutrinos scattering with the galactic DM halo for which they set limits of $\sim$ $10^{-22}(\mathrm{~m} / \mathrm{GeV})(\mathrm{E} / \mathrm{PeV})^{2} \mathrm{~cm}^{2}$ [107]. Both of these constraints assumed the cross sec-

tion can be modeled as $\sigma \sim \sigma_{0}\left(\frac{m_{\chi}}{\mathrm{GeV}}\right)\left(\frac{E}{E_{0}}\right)^{2}$. This can be compared with Eq. (4.8), where this approximation is seen to no longer hold when the centre-of-mass energy of the neutrino approaches the mediator mass. This is the case for high mediator masses $(\mathrm{GeV}-\mathrm{PeV})$. The limits from neutrinos scattering with extragalactic DM assumed that a time delay from neutrino scattering would lead to a cut-off in the spectrum at a specific energy that is dependent on cross section. The limits from neutrinos scattering with the Milky Way DM halo assumed that the scattering interaction would lead to an excess of neutrinos in the Galactic Centre. This excess was the result of neutrinos appearing to originate from the Galactic Centre, even if they come from outside the Milky Way, due to increased scattering at the Galactic Centre. While they accounted for an increase in neutrinos that appear to originate from the Galactic Centre they did not seem to consider the reduction that should also be expected. This work on the spectral variation also lacked sufficient observational data that has now become available, as well as the proper techniques to model the atmospheric background [107]. 


\subsection{DARK MATTER-NEUTRINO SCATTERING}

\subsubsection{Previous HESE work}

Previous work has been done to study DM-neutrino scattering in the Galactic Centre with the four year high energy starting event (HESE) dataset from IceCube contain-

ing 53 events [1]. They were able to find that constraints from IceCube were more stringent than those from cosmology for specific regions of the DM, mediator, and coupling parameter space. There is now more data that exists from IceCube that can be used to improve their bounds, as well as other DM-neutrino models that can be considered.

\subsubsection{Cascade Equation}

This analysis considers extragalactic neutrinos travelling towards the Earth that scatter with the diffuse DM halo of the Milky Way. Since the DM column density is different for different arrival directions, there is expected to be a shadow in the neutrino flux in the direction of the Galactic Centre. The changes in the neutrino flux that are a result of the DM-neutrino scattering are characterized by the cascade equation:

$$
\frac{d \Phi(E, \tau)}{d \tau}=-\sigma(E) \Phi(E, \tau)+\int_{E}^{\infty} d \tilde{E} \frac{d \sigma(\tilde{E}, E)}{d E} \Phi(\tilde{E}, \tau)
$$

where $\Phi$ is the neutrino flux, $E$ is the neutrino energy, $\tau$ is the DM column density, and $\sigma$ is the scattering cross section. The first term accounts for loses due to scattering interactions, while the second term accounts for the addition of neutrinos scattering from higher energies to lower energies [70].

The column density describes the amount of DM along the l.o.s. to the neutrino 
source:

$$
\tau(\vec{x})=\int_{l . o . s} n_{\chi}(\vec{x}) d x
$$

For simplicity, the distance $x$ over which the DM number density is integrated is converted to galactic coordinates. This is done via the transformation:

$$
r=\sqrt{x^{2}+R^{2}-2 x R \cos (\theta)}
$$

where $r$ is the distance to the Galactic Centre, $x$ is the distance from the Solar system, $R$ is the distance from the Sun to the Galactic Centre $R=8.3 \mathrm{kpc}$, and $\theta$ is the angle to the Galactic Centre.

\subsubsection{Other Considerations}

\section{Scattering angle}

An important consideration in this analysis is that the scattering angle be small so that the neutrinos will lose energy, but not change direction significantly as they pass through the galactic dark matter halo. The differential cross section $\frac{d \sigma}{d \cos \theta}$ from Eq. (4.7) is plotted against scattering angle $\theta$ in Fig. 4.2. It is seen that the smallest angles $(\cos \theta \sim 1)$ are associated with a strong peak in the differential cross section indicating the preference for small scattering angles. This means that the angular spread in DM-neutrino scattering signal from the Galactic Centre would not be effected by the scattering angle. Instead this spread is determined by the DM halo profile. 


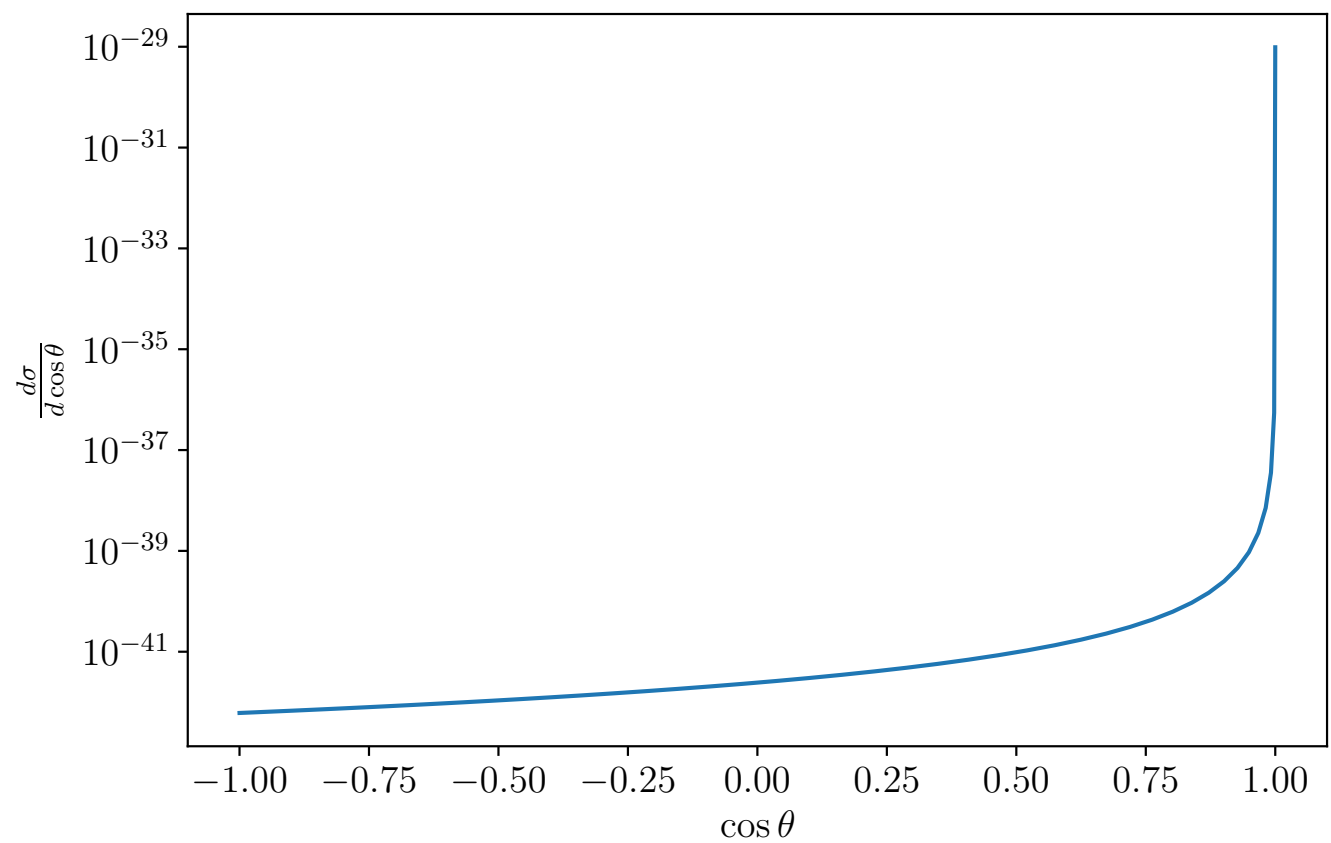

Figure 4.2: The angular dependence of the differential cross section. Small scattering angles are strongly favoured indicating that after DM-neutrino scattering the neutrino maintains is original direction.

\section{Combined Dark Matter-Neutrino Scattering and Annihilation}

Analyzing neutrino flavours observed at IceCube could provide deeper insight into possible flavour dependent dark matter neutrino interactions. Constraining much of these interactions requires stronger statistics than presently available [111-114]

\section{Combined Dark Matter-Neutrino Scattering and Annihilation}

For neutrino-DM scattering it could be possible to contrive a scenario where the deficit in the neutrino flux from scattering could be balanced by the excess from annihilation or decay. Such a scenario would be difficult to design since the annihilation and 


\subsection{DARK MATTER-NEUTRINO SCATTERING}

scattering signals require different DM mass ranges. Based on current constraints the DM mass range that can lead to significant amounts of scattering is sub-GeV [1], while the mass range of DM that annihilates to neutrinos is in the $\mathrm{PeV}$ [93].

\section{Extragalactic Dark Matter Scattering}

A simple case of DM-neutrino scattering with the isotropic extragalactic DM can also be considered. In the case of DM-neutrino scattering outside of the Milky Way, the DM redshifts as:

$$
\rho_{\chi}(z)=\rho_{\chi_{0}}(1+z)^{3}
$$

where $\rho_{\chi_{0}}=1.25 \mathrm{GeV} / \mathrm{cm}^{3}$ is the present DM density [4] and $z$ is the redshift.

By computing the comoving distances of the DM with Ref: [115] and calculating the column density, the contributions from the Milky Way DM halo can be compared with the extragalactic DM. As shown in Fig. 4.3 the galactic component dominates for all but the highest redshifts. Since the most distant astrophysical objects have redshifts $\sim 10^{1}$, the extragalactic DM-neutrino scattering can be neglected [116]. 


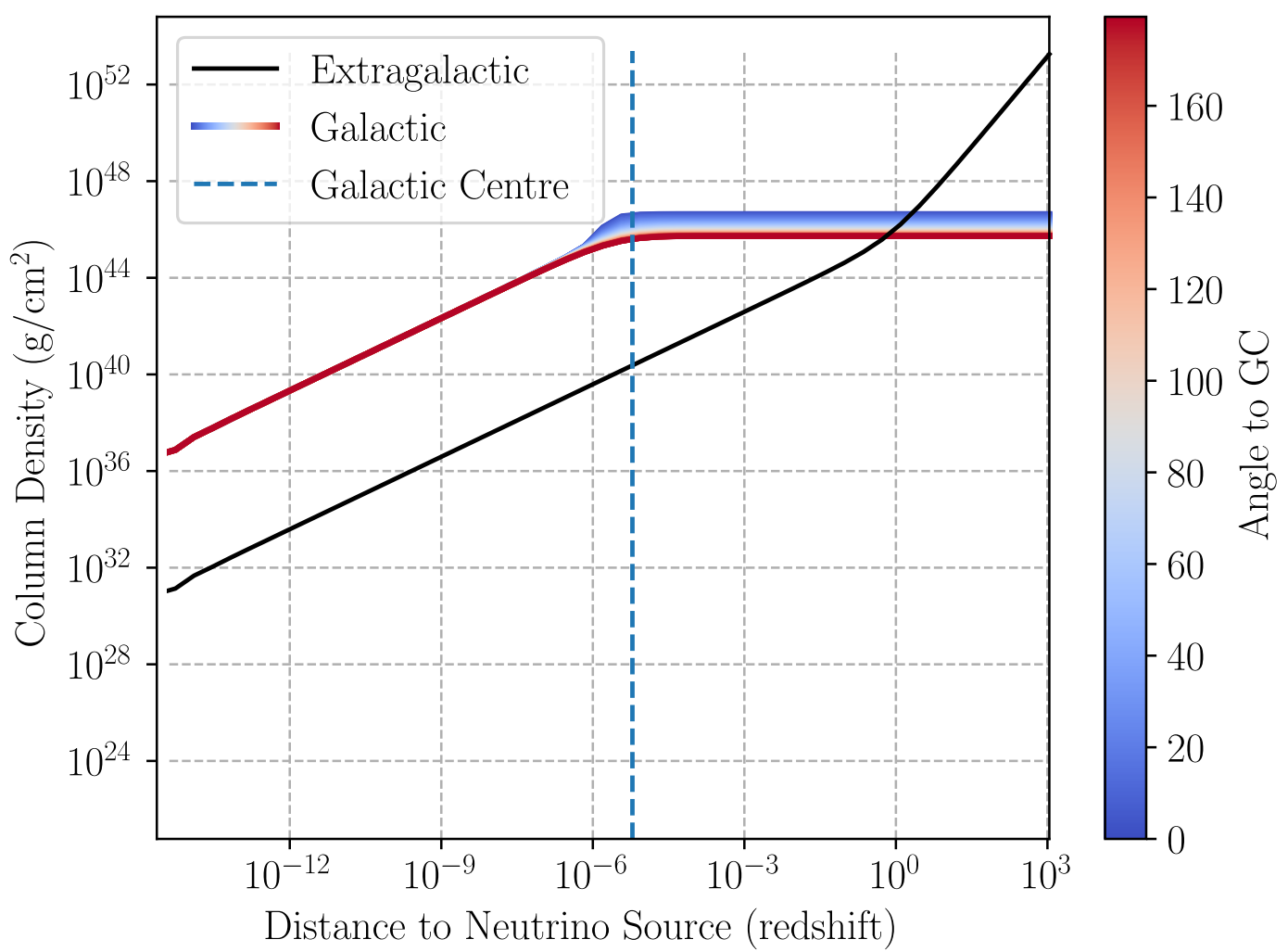

Figure 4.3: A comparison of the extragalactic and galactic contributions to the DM column density. Here the distance to the neutrino source is the length from Earth over which the column density is integrated ( $x$ in Eq. (4.4)). The extragalactic component is modelled with the redshifted DM from (4.6), while the galactic component is modelled using the NFW halo described by (2.11). The line for the Galactic Centre represents the comoving distance to the Galactic Centre converted to a redshift. The contribution to dark matter column density from the Milky Way halo dominates up to redshifts $\sim 1$ where the contribution from relic dark matter outside the Milky Way. It can also be seen that the angles nearest to the galactic centre have higher column densities.

\subsubsection{Dark Matter-Neutrino Scattering Models}

Four DM-neutrino elastic scattering scenarios are considered for this thesis. The Feynman diagrams for these processes are shown in Fig. 4.4. In these models particle 


\subsection{DARK MATTER-NEUTRINO SCATTERING}

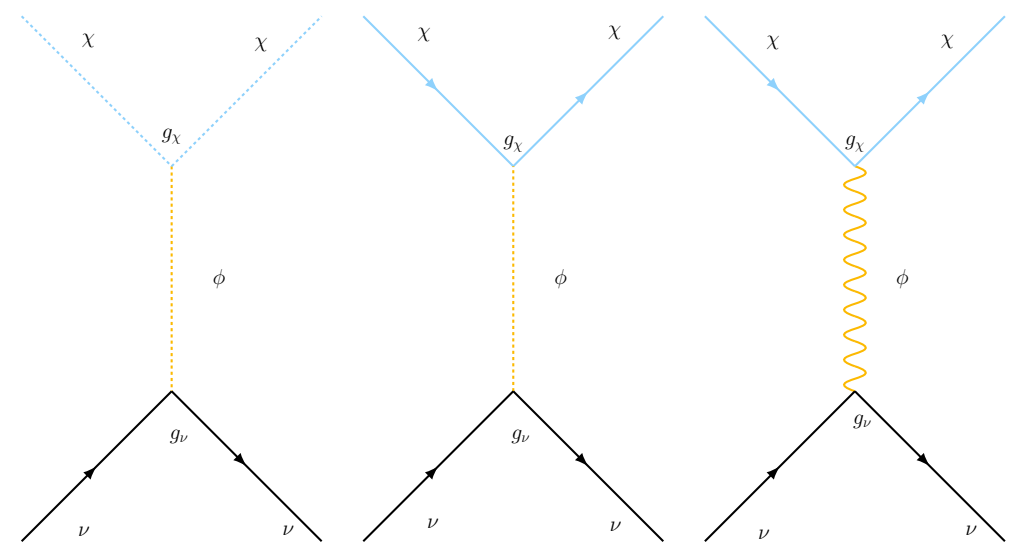

(a) Scalar DM scalar (b) fermion DM (c) Fermion DM vecmediator scalar mediator tor mediator
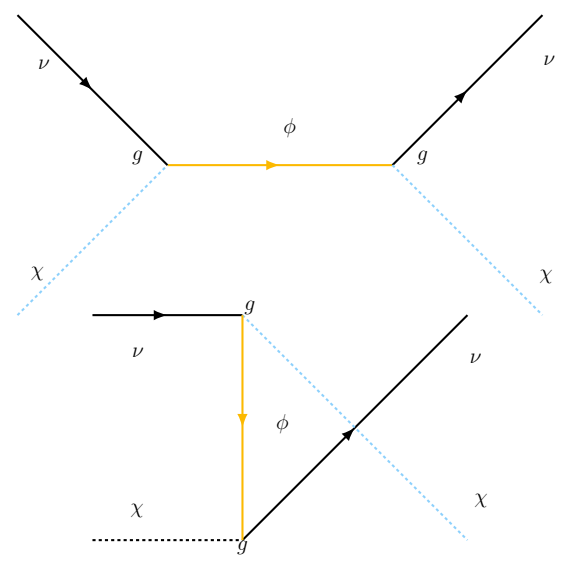

(d) Scalar DM fermion mediator

Figure 4.4: Feynman diagrams of DM-neutrino scattering models.

$\chi$ is the DM particle and $\phi$ is the mediator, which are connected by the coupling $g$. The cross sections are derived assuming that the neutrino mass is negligible, the DM is massive, no flavour effects, elastic scattering, and no differences between neutrinos and anti-neutrinos. The four models are summarized as:

- Scalar mediator-scalar DM: the neutrino coupling $g_{\nu}$ is similar to a Yukawa coupling and is dimensionless while the $g_{\chi}$ has dimensions of energy. The Lagrangian has the term $\mathcal{L}_{\text {int }}=-g_{\nu} \phi \bar{\nu} \nu-g_{\chi} \phi \chi^{2}+$ h.c.. 


\subsection{DARK MATTER-NEUTRINO SCATTERING}

- Scalar singlet mediator-dirac fermion DM: higgslike mediator. The Lagrangian has the term $\mathcal{L}_{i n t}=-g_{\nu} \phi \bar{\nu} \nu-g_{\chi} \phi \bar{\chi} \chi+$ h.c..

- Vector mediator-dirac fermion DM: like a new leptophillic Z' gauge boson. It scales with $E$. The Lagrangian has the term $\mathcal{L}_{i n t}=-g_{\chi_{L}} \bar{\chi}_{R} \gamma^{\mu} Z_{\mu}^{\prime} \chi_{L}-$ $g_{\chi_{R}} \bar{\chi}_{R} \gamma^{\mu} Z_{\mu}^{\prime} \chi_{R}-g_{\nu} \bar{\nu}_{L} \gamma^{\mu} Z_{\mu}^{\prime} \nu_{L}$

- Fermion mediator-scalar DM: inspired by right handed sneutrino models-it has a s-channel diagram and resonant structure $\mathcal{L}_{i n t}=-g \chi \bar{\phi} \nu_{L}+$ h.c..

In these scenarios elastic scattering can be expected as the DM is not assumed to be composite. This scattering rate is proportional to the DM column density and inversely proportional to the DM mass. It is assumed that the DM particle is at rest and that $m_{\chi} \ll E_{\nu}$. This leads to this reaction being favoured for sub-GeV DM. In this thesis the scalar DM-scalar mediator model was selected to be studied as it is the simplest and has not been previously constrained at IceCube. The scattering cross sections that are used from Ref: [1] are shown in Fig. 4.5 for the case of a scalar DM and scalar mediator, with a coupling of $g^{2}=1$. In the case of the scalar DM-scalar mediator the differential cross section is:

$$
\frac{d \sigma}{d \cos \theta}=\frac{g_{\nu}^{2} g_{\chi}^{2}(1-x) E_{\nu_{i}}^{2} m_{\chi}}{16 \pi\left((1-x) E_{\nu_{i}}+m_{\chi}\right)\left((1-x) E_{\nu_{i}} m_{\phi}^{2}+m_{\chi}\left(m_{\phi}^{2}-2(x-1) E_{\nu_{i}}^{2}\right)\right)^{2}},
$$

which is integrated to give the total cross section:

$$
\sigma=-g_{\nu}^{2} g_{\chi}^{2} \frac{4 E_{\nu_{i}}^{2} m_{\chi}+\left(2 E_{\nu_{i}} m_{\phi}^{2}+4 E_{\nu_{i}}^{2} m_{\chi}+m_{\phi}^{2} m_{\chi}\right) \log \left(\frac{m_{\phi}^{2}\left(2 E_{\nu_{i}}+m_{\chi}\right)}{2 E_{\nu_{i}} m_{\phi}^{2}+4 E_{\nu_{i}}^{2} m_{\chi}+m_{\phi}^{2} m_{\chi}}\right)}{64 \pi E_{\nu_{i}}^{2} m_{\chi}^{2}\left(2 E_{\nu_{i}} m_{\phi}^{2}+4 E_{\nu_{i}}^{2} m_{\chi}+m_{\phi}^{2} m_{\chi}\right)}
$$

Here $E_{\nu_{i}}$ is the initial neutrino energy, $m_{\chi}$ is the DM mass, $m_{\phi}$ is the mediator mass, 
$g_{\nu}$ and $g_{\chi}$ are the couplings, $x=\cos \theta$, and $\theta$ is the scattering angle. The cross sections of the other models are provided in Appendix A.

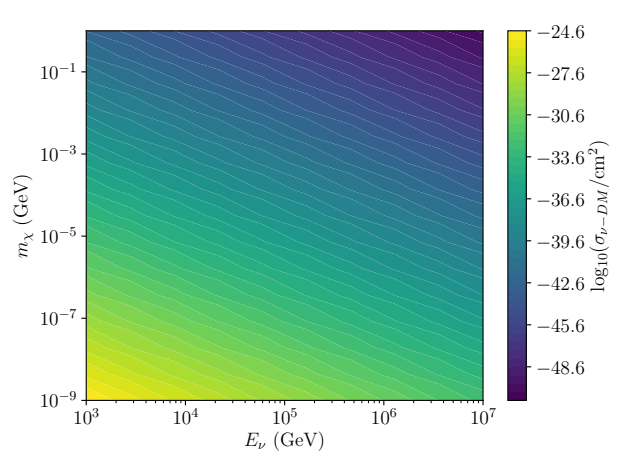

(a)

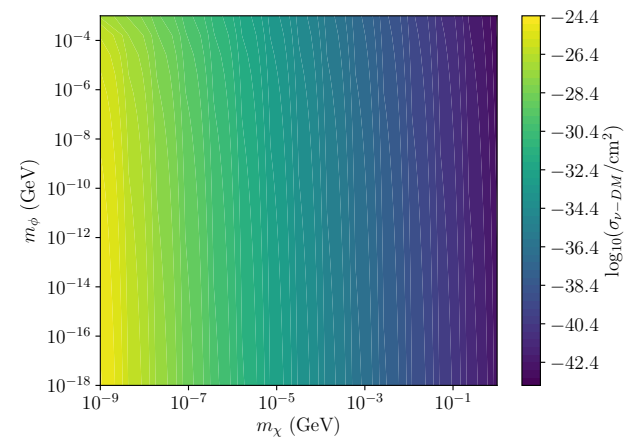

(b)

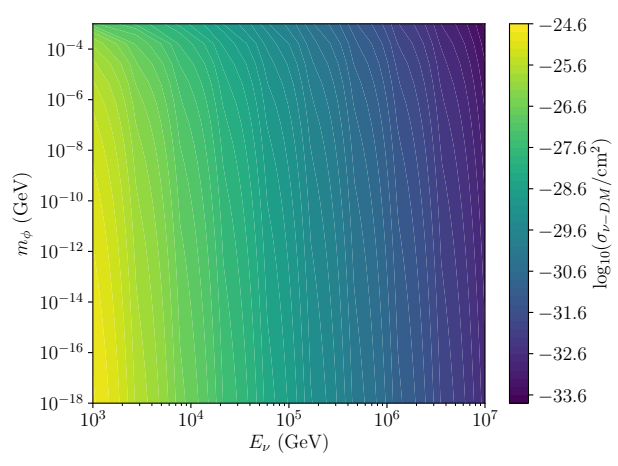

(c)

Figure 4.5: DM-neutrino scattering cross sections for the scalar DM-scalar mediator model. Here the coupling is $g=1$, and the fixed variables are a) $m_{\phi}=10^{2}$ $\mathrm{eV}$, b) $E=10^{3} \mathrm{GeV}$, c) $m_{\chi}=10^{6} \mathrm{eV}$.

\subsubsection{Dark Matter-Neutrino Interactions in Cosmology}

While $\Lambda \mathrm{CDM}$ cosmology assumes that DM and neutrinos are non-interacting, possible interaction scenarios could have effects on the angular power spectra of the CMB, the matter power spectrum and large scale structure. The Early Universe is a prime 


\subsection{DARK MATTER-NEUTRINO SCATTERING}

candidate for exploring the weak interactions of neutrinos and DM, since the number densities were much greater in the past. The DM density scales with redshift $z$ as $\rho_{D M} \propto(1+z)^{3}$ and the neutrino density in the Universe goes as $\rho_{\nu} \propto(1+z)^{4}$ $[117,118]$.

In possible neutrino-DM scattering scenarios, DM remains in kinetic connect with neutrinos long after freeze-out. In this case, DM will not cluster under gravity because collisional damping will lead to less small-scale DM perturbations [98]. Simultaneously neutrinos are no longer free streaming, and will cause an enhancement in the CMB acoustic peaks and reduction in small-scale power in the matter power spectrum [98]. These effects are contained as a drag term in the perturbed Euler

equations. In the Newtonian gauge assuming a flat Universe, the density and velocity perturbation equations are given by [106]:

$$
\begin{aligned}
\dot{\delta}_{D M} & =-\theta_{D M}+3 \dot{\phi}, \\
\dot{\theta}_{D M} & =k^{2} \psi-\mathcal{H} \theta_{D M}-S^{-1} \dot{\mu}\left(\theta_{D M}-\theta_{\nu}\right), \\
\dot{\delta}_{\nu} & =-\frac{4}{3} \theta_{D M}+4 \dot{\phi}, \\
\dot{\theta}_{\nu} & =k^{2} \psi+k^{2}\left(\frac{1}{4} \delta_{\nu}-\sigma_{\nu}\right)-\dot{\mu}\left(\theta_{D M}-\theta_{\nu}\right),
\end{aligned}
$$

where $\delta_{D M}$ and $\delta_{\nu}$ are the DM and neutrino density perturbations, $\theta_{D M}$ and $\theta_{\nu}$ are velocity divergences, $k$ is the comoving wavenumber, $\psi$ the gravitational potential, $\sigma_{\nu}$ is the neutrino ansiotropic stress potential, and $\mathcal{H}$ is the conformal Hubble parameter. The parameter of interest here is the the DM-neutrino scattering rate $\dot{\mu}=a \sigma_{D M-\nu} n_{D M}$, which can be used to constrain the neutrino-DM cross section. 


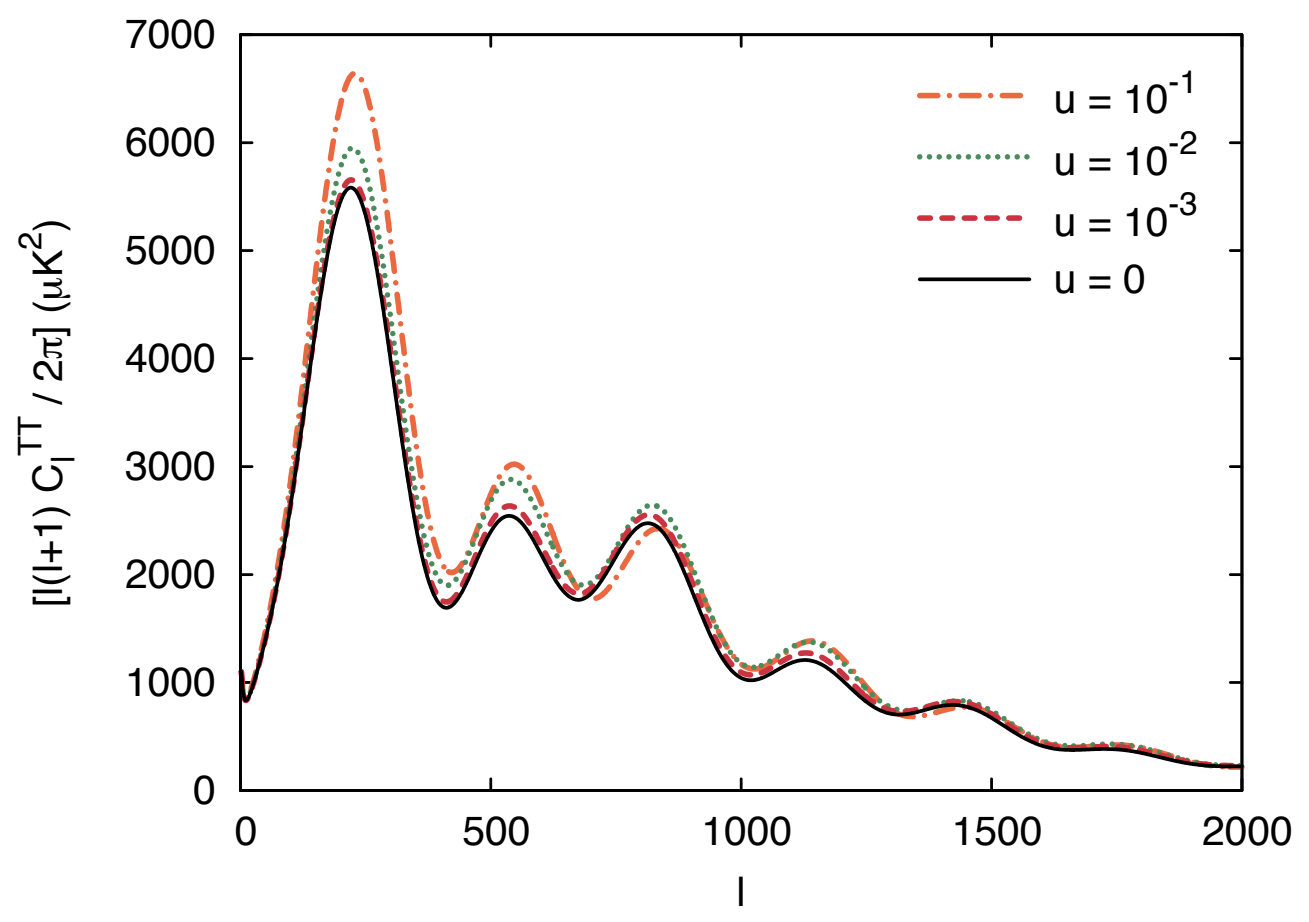

Figure 4.6: CMB angular power spectrum for varying effects of a neutrino-DM interaction cross section. Fig. from Ref: [15].

$\dot{\mu}$ is typically expressed as a dimensionless constant:

$$
u=\left(\frac{\sigma_{\nu}}{\sigma_{T h}}\right)\left(\frac{m_{\chi}}{100 \mathrm{GeV}}\right)^{-1}
$$

The effects of varying neutrino-DM cross sections on the CMB angular power spectrum are shown in Fig. 4.6.

For this case of large scale structure upper limits on the DM-neutrino scattering 
cross section have been set as:

$$
\sigma_{D M-\nu} \lesssim 10^{-33}\left(m_{\chi} / \mathrm{GeV}\right) \mathrm{cm}^{2}
$$

for the s-wave and

$$
\sigma_{D M-\nu} \lesssim 10^{-45}\left(\frac{T}{T_{0}}\right)^{2}\left(m_{\chi} / \mathrm{GeV}\right) \mathrm{cm}^{2}
$$

for the p-wave case were obtained by [15].

These interactions have also been considered in terms of the effects on the growth of small structures from elastic scattering between DM and photons which would lead to a suppression of small-scale structures [96]. This was done by comparing the number of satellite galaxies in the Milky Way with what would be expected for a weak DM-photon interaction hypothesis. An estimate on the upper limit on the elastic scattering cross section as $\sigma_{\nu D M}<10^{-35}\left(m_{D M} / \mathrm{GeV}\right)$. This work hasn't been confirmed and was an estimate extrapolated from bounds on DM-photon interactions [96].

\subsubsection{Comparing IceCube constraints with Cosmology}

While the constraints from IceCube and cosmology apply to different neutrino energy ranges ( $\mathrm{TeV}$ vs $\lesssim \mathrm{eV}$ ), the constraints can be compared for a specific model by taking the low-energy limit of the neutrino-DM cross section. In the low energy limit the cross sections are found by expanding a power series in $E_{\nu}$ about $E_{\nu}=0$. These limits are: 
Scalar DM-scalar mediator:

$$
\sigma_{L E}=\frac{g_{\phi}^{2} g_{\chi}^{2} E_{\nu}^{2}}{8 \pi m_{\chi}^{2} m_{\phi}^{4}}
$$

Fermion DM-scalar mediator and fermion DM-vector mediator:

$$
\sigma_{L E}=\frac{g_{\phi}^{2} g_{\chi}^{2} E_{\nu}^{2}}{2 \pi m_{\phi}^{4}}
$$

The cosmology limits have both a p-wave and s-wave component from an $T^{2}$ expansion to the cross section from the Boltzmann equation:

$$
\sigma \propto a T^{0}+b T^{2}
$$

In this case the best limits from cosmology for the coefficients $a$ and $b$ are from Eqs. (4.14) and (4.15), while the temperature evolves as:

$$
T_{\nu}(z)=T_{\nu, 0}(1+z) \approx 1.68 \times 10^{-4}(1+z) \mathrm{eV},
$$

here a redshift at recombination of $z=1089$ and present temperature of $T_{0, \nu} \simeq$ $1.68 \times 10^{-4} \mathrm{eV}$ was used [117]. At recombination neutrinos can be assumed to follow a Fermi-Dirac distribution with energy $E_{\nu}$ and temperature $T$ [119]:

$$
f\left(E_{\nu}, T\right)=\frac{1}{e^{\frac{E_{\nu}}{k_{B} T}}+1},
$$

where $k_{B}$ is the Boltzmann constant. 


\subsection{DARK MATTER-NEUTRINO SCATTERING}

The average neutrino energy can be found as:

$$
\left\langle E_{\nu}\right\rangle=\frac{\int_{0}^{\infty} E_{\nu} f\left(E_{\nu}, T\right) d E_{\nu}}{\int_{0}^{\infty} f\left(E_{\nu}, T\right) d E_{\nu}} .
$$

Taking $T / T_{0}=(1+z)$ and the average energy is given as $\left\langle E_{\nu}\right\rangle \approx k_{B} T \approx 0.2 \mathrm{eV}$ then the cosmological limits constrain the coupling as:

Scalar-scalar:

$$
g_{\chi} g_{\phi} \lesssim 1.5 \times 10^{7}\left(\frac{m_{\phi}^{4} m_{\chi}^{3}}{\mathrm{GeV}^{7}}\right)^{1 / 2} \mathrm{GeV}
$$

Fermion DM-scalar mediator and fermion DM-vector mediator:

$$
g^{2} \lesssim 5.3 \times 10^{6}\left(\frac{m_{\phi}^{4} m_{\chi}}{\mathrm{GeV}^{5}}\right)^{1 / 2}
$$




\section{Chapter 5}

\section{Method}

This analysis aimed to set sensitivities in searches for an energy dependent deficit in the isotropic extra-galactic neutrino flux at the Galactic Centre from DM neutrino elastic scattering. This was done using an unbinned likelihood analysis with simulated IceCube data using a Markov Chain Monte Carlo (MCMC) algorithm. This involved determining an expected signal by combining a DM hypothesis with models for astrophysical and atmospheric neutrinos and muons. A likelihood was then constructed to compare this expected signal with a simulated dataset. This likelihood was then explored using a MCMC to constrain the upper limits on DM parameters and allow the nuisance parameters to be included as free parameters.

\subsection{MESE-C Dataset}

In this thesis, a Monte Carlo simulated sample for the IceCube medium energy starting event-cascades (MESE-C) data is used. Since the IceCube data unblinding process for observational data for this analysis is still underway, the full dataset will be analyzed in a future paper. As a result, this thesis uses a simulated sample dataset that is constructed by randomly selecting events from the Monte Carlo simulation of 


\begin{tabular}{|l||l|l|}
\hline Parameter & MESE-C & HESE \\
\hline Energy $(\mathrm{GeV})$ & $1 \mathrm{TeV}-10^{4} \mathrm{TeV}$ & $30-10^{4} \mathrm{TeV}$ \\
$N_{\text {astro }}$ & 551 & 34 \\
$N_{\text {atm }}$ & 1369 & 14 \\
$N_{\text {muon }}$ & 57 & 7 \\
$N$ & 1976 & 54 \\
\hline
\end{tabular}

Table 5.1: Comparison of the HESE [1] and MESE-C [2] datasets. The number of each type of event are based on fits to models described in Sec. 3.6.

expected events for the MESE-C data.

As described in the seven year MESE-C paper [2], the MESE-C dataset contains 1980 events across seven years $(209,779,200$ seconds) of data taking. These detected events have energies from $1 \mathrm{TeV}$ to $10 \mathrm{PeV}$ over the full sky. Of these, 550 are expected to be astrophysical, 1300 atmospheric, and 45 muons [2]. Through an improved veto technique, a lower energy threshold can be used compared to the previous high energy starting event (HESE) data, so that a greater number of astrophysical neutrinos can be observed. This marks a notable increase in the number of astrophysical events over the previously used 4-year HESE dataset, which contained 54 events. This improvement comes at the cost of an increased number of atmospheric background events to model. A comparison of these datasets is shown in Table 5.1. Since IceCube is located at the South Pole, certain veto methods like using the Earth as a shield are not possible when observing the Galactic Centre which is located in the southern sky. The Galactic Centre is located at RA 17h 45m 40.04s, Dec -29 00' 28.1" [120]. The MESE-C data was optimal for this analysis as its veto method allow for observations of events from the entire sky [2],.

The distribution of events for the MESE-C observational dataset is shown in Fig. 5.1 across energy and declination. The coloured histogram is the Monte Carlo 
expectation separated by the event source (muon, atmosphere, astrophysical), while black crosses are the observed data. The dataset was consistent with an isotropic extragalactic flux with a $\left(\nu_{e}, \nu_{m} u, \nu_{t} a u\right)=(1: 1: 1)$ flavour ratio. The observed data can be compared with 5.2 which shows the distribution of a simulated sample dataset that is used to take the place of real data. This simulated dataset was constructed by randomly sampling from the Monte Carlo expectation. The Monte Carlo expectation is shown in colour bars for each component of the flux. The simulated sample can be considered to provide a reasonable replacement of the real data. The angular resolution is provided by Ref: [2] and shown in Fig. 5.3

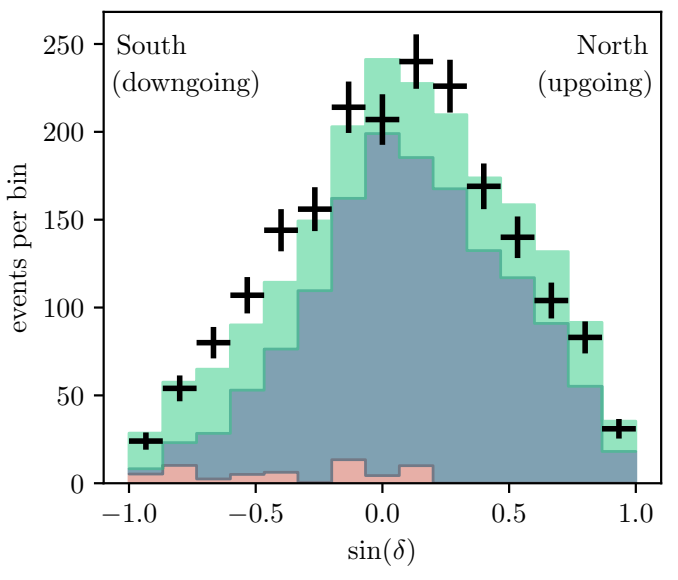

(a)

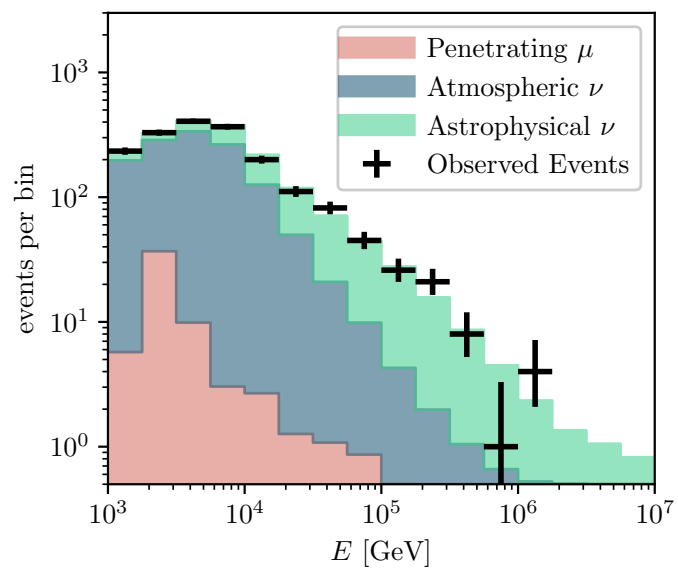

(b)

Figure 5.1: Distribution of the observed MESE-C events (black crosses) in a) declination and b) energy. The coloured histogram shows the Monte Carlo expectation for each event's expected source (muon, atmosphere, astrophysical). Figure from Ref: [2] 


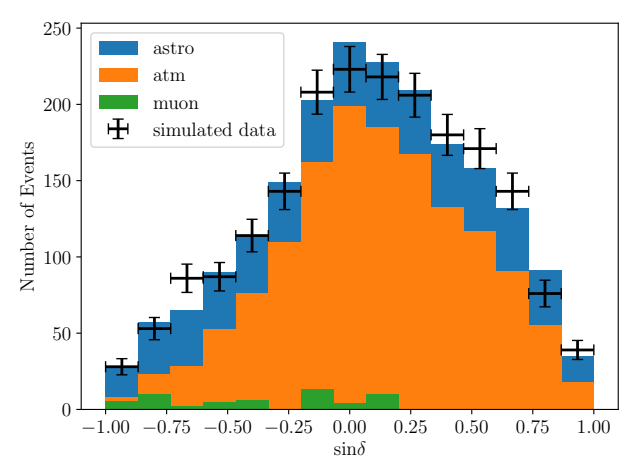

(a)

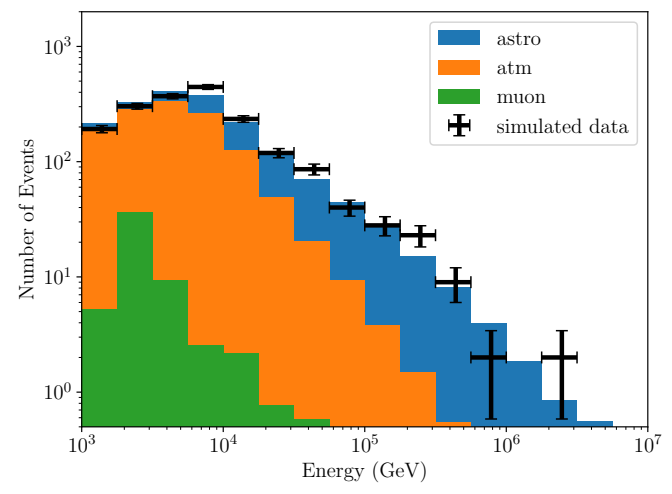

(b)

Figure 5.2: Distribution of the Monte Carlo randomly sampled simulated sample events in energy and declination alongside the Monte Carlo expectation of each source.

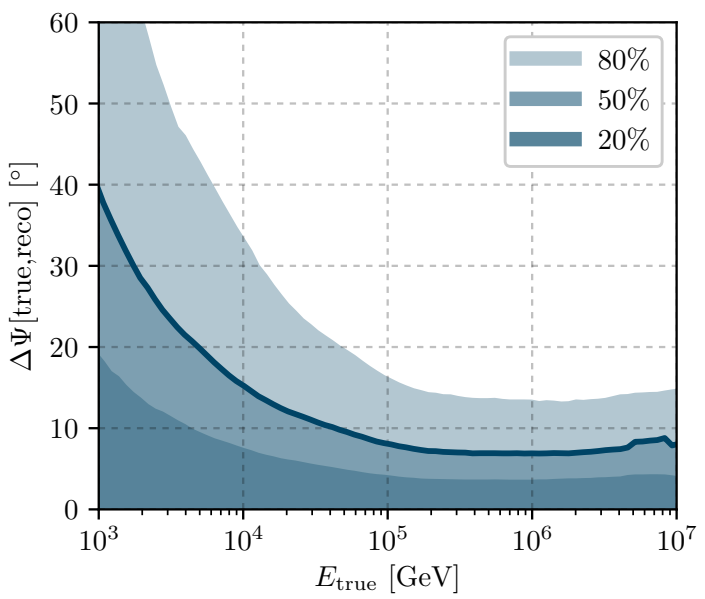

Figure 5.3: The angular resolution of the MESE-C dataset. The low energy neutrinos have a high angular error. Figure from Ref: [2]. 


\subsubsection{Monte Carlo Simulated Data}

A Monte Carlo simulation was used to determine the expected neutrino flux and probability density functions for the events that are expected in IceCube. This was constructed with the NuGen code. The NuGen Monte Carlo software has recently been superseded by the LeptonInjector and LeptonWeighter packages [121]. A separate Monte Carlo set was used for the expected number of muons that were simulated using muongun, which is an IceCube implementation of MUPAGE: [122].

The Monte Carlo simulation was then adjusted with the nuVeto software to account for the veto region in the detector that was used to reduce the number of atmospheric neutrinos [123].

Numerous reconstruction techniques can be used to approximate a neutrinos energy and direction based on the light that is detected in the IceCube detectors. These are shown in Fig. 5.4. For this thesis the 'true' neutrino energy and direction are used for the neutrino source models, while the probability density functions used in the likelihood described in Sec. 5.3 use the simulated reconstructed values.

\section{OneWeight}

An important part of observing events at IceCube is understanding the physics of the Antarctic ice and how dust and sediment affects the transmission of light $[124,125]$. When constructing a Monte Carlo of expected events effects including ice properties, the neutrino-nucleon cross section, and DOM efficiency. These effects are contained in the weighting that is assigned to each Monte Carlo event to describe the probability that a given neutrino will be observed at the detector [126]. This weight is produced 


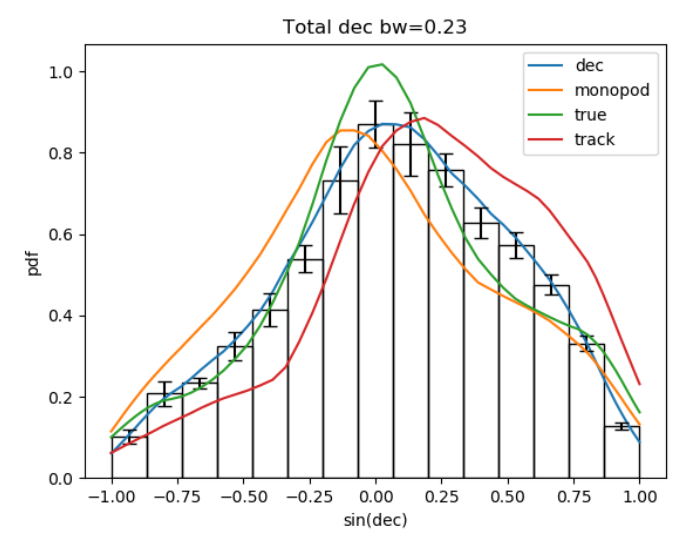

Figure 5.4: The different reconstruction methods that were used in [12]. These are only shown for the Monte Carlo simulation, where true is the declination that represents the neutrino energy at an astrophysical source.

alongside the Monte Caro simulation from NuGen and is called the OneWeight:

$$
\text { OneWeight }=\frac{P_{\text {int }}}{E^{-\gamma}} \int_{E_{\text {min }}}^{E_{\max }} E^{-\gamma} d E \cdot \text { Area } \cdot \Omega \cdot T \mathrm{GeV} \mathrm{cm}{ }^{2} \mathrm{~s} \mathrm{sr}
$$

where $P_{\text {int }}$ is the total interaction probability weight, $E^{-\gamma}$ the neutrino energy generation shape, $E_{\min }$ and $E_{\max }$ the minimum and maximum energies of the neutrinos. Area is the detector area, $\Omega$ is the generation solid angle, and $T$ is the detector's live time.

The interaction probability is given as:

$$
P_{\text {int }}=1-P_{\text {surviving }}=1-e^{-\sigma_{\text {tot }} L_{d} / M_{p}} .
$$

where $\sigma_{\text {tot }}$ is the total cross section at the interaction point, $L_{d}$ is the total column depth, and $M_{p}$ is the proton mass [127]. 


\subsection{DARK MATTER HYPOTHESIS}

The weight of a given flux is modified by the OneWeight as:

$$
w_{i}=\frac{\text { OneWeight }}{N} \frac{d \Phi_{\nu}\left(E_{\nu}\right)}{d E_{\nu}}
$$

where $N$ is the number of events and $\frac{d \Phi_{\nu}\left(E_{\nu}\right)}{d E_{\nu}}$ is the neutrino flux.

The expected number of events for the Monte Carlo is found as:

$$
N=\frac{d \Phi_{\nu}\left(E_{\nu}\right)}{d E_{\nu}} * \text { OneWeight } * L
$$

where $L$ is the detector's live time.

\subsection{Dark Matter Hypothesis}

The DM models described in Sec. 4.3.4 are used to model the interaction cross section, while the NFW DM profile described in Sec 2.3 is used to calculate the DM column density. These are incorporated into the cascade equation from Eq. (4.3). The code that is used to solve the cascade equation is based on nuFATE (neutrino fast attenuation through Earth) [70], which was designed to efficiently model the attenuation of neutrinos passing through the Earth. In this thesis nuFATE was modified in Python to include a DM halo, and DM-neutrino interactions. The code works by simplifying the cascade equation with the substitution $\varphi(E) \equiv d \phi_{\nu_{\ell}} / d E$ :

$$
\frac{d \varphi(E)}{d x}=-\sigma(E) \varphi(E)+\int_{E}^{\infty} d \tilde{E} f(E, \tilde{E}) \varphi(\tilde{E})
$$

The nuFATE code then quickly solves this equation by vectorizing the energy and neutrino flux: $E_{\nu} \rightarrow \vec{E}_{\nu}, \varphi \rightarrow \vec{\phi}$. 


\subsection{DARK MATTER HYPOTHESIS}

The cascade equation then takes the form of an eigenvalue problem:

$$
\frac{d \vec{\phi}}{d x}=(-\operatorname{diag}(\vec{\sigma})+C) \vec{\phi}=M \vec{\phi}
$$

The components of the matrix $C$ are from the integrand in Eq. 5.5: $C_{i j}=f\left(E_{i}, \tilde{E}_{j}\right)$. Since Eq. (5.6) is linear, the eigenvectors $\hat{\phi}_{i}$ of $M$ individually satisfy the differential equation $\hat{\phi}_{i}^{\prime}=\lambda_{i} \hat{\phi}_{i}$, where $\lambda_{i}$ are the corresponding eigenvalues. The solution to Eq. (5.6) is thus

$$
\vec{\phi}=\sum c_{i} \hat{\phi}_{i} e^{\lambda_{i} x}
$$

The coefficients $c_{i}$ are determined by the initial $(x=0)$ neutrino flux, which corresponds to a power law for the astrophysical flux as described in Sec. 3.6.1. This solution is vectorized across energy and the flux is interpolated in Python to obtain a continuous DM-adjusted astrophysical flux:

$$
\frac{d \Phi_{\text {astro }}}{d E_{\nu}}=\operatorname{interp}(\vec{\phi})
$$

An example of the column density for the NFW halo profile is shown in Fig. 5.5, where the Galactic Centre has a higher column density. A Burkert or cored profile would be expected to produce a lower signal that is more spread out across the sky. Since the likelihood of a scattering interaction is proportional to the column density a greater scattering effect should be expected at the Galactic Centre. This scattering effect would attenuate the high energy neutrino flux as the neutrinos lose energy. Fig. 5.6 shows the skymap of the flux survival probability for a scalar DMscalar mediator model, where the astrophysical neutrino flux would be expected to be reduced at the Galactic Centre. 


\subsection{DARK MATTER HYPOTHESIS}

This survival probability across energy and angle to the Galactic Centre is shown in Fig. 5.7. For this specific example of a scalar DM and scalar mediator there is a noticeable attenuation for energies below $1 \mathrm{PeV}$. There is also a gradient in attenuation where l.o.s angles closer to the Galactic Centre (smaller) lead to lower survival probability for any given energy. Here the advantage of MESE-C data including lower energies becomes apparent, as the higher energy HESE wouldn't be able to probe this interaction as well.

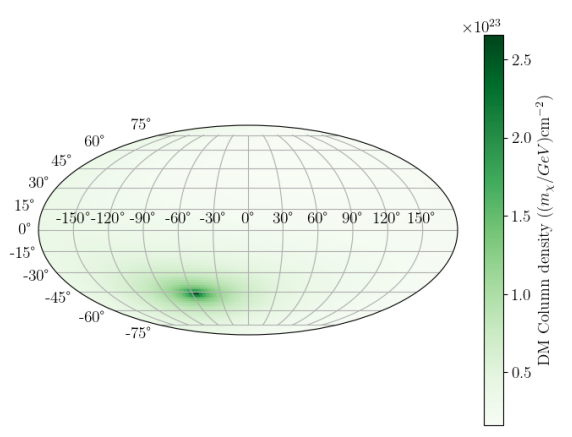

(a) NFW

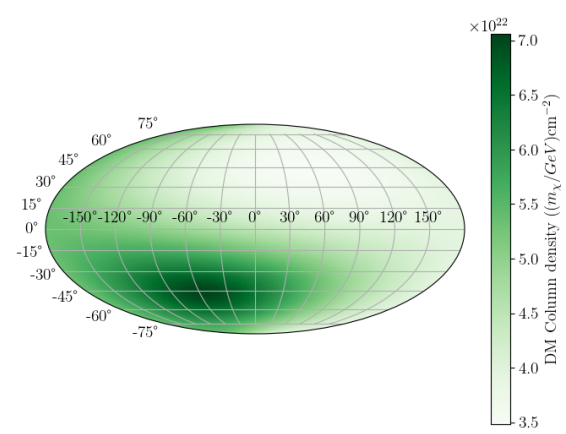

(b) Burkert

Figure 5.5: Skymap of the DM column density. The column density increases at the Galactic Centre. 


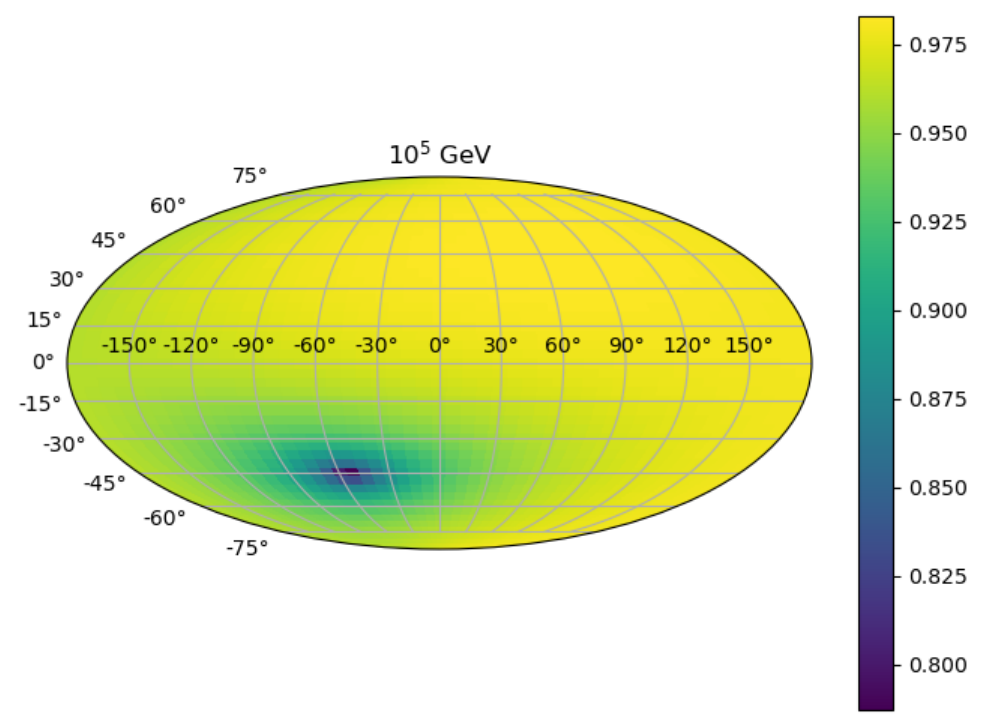

Figure 5.6: Skymap of the survival probability for the astrophysical neutrino flux. This is for the scalar mediator-scalar DM scenario with $g=1, m_{\phi}=10^{6}$ $\mathrm{MeV}, m_{\chi}=1 \mathrm{GeV}$ at $E_{\nu}=1 \mathrm{PeV}$. 


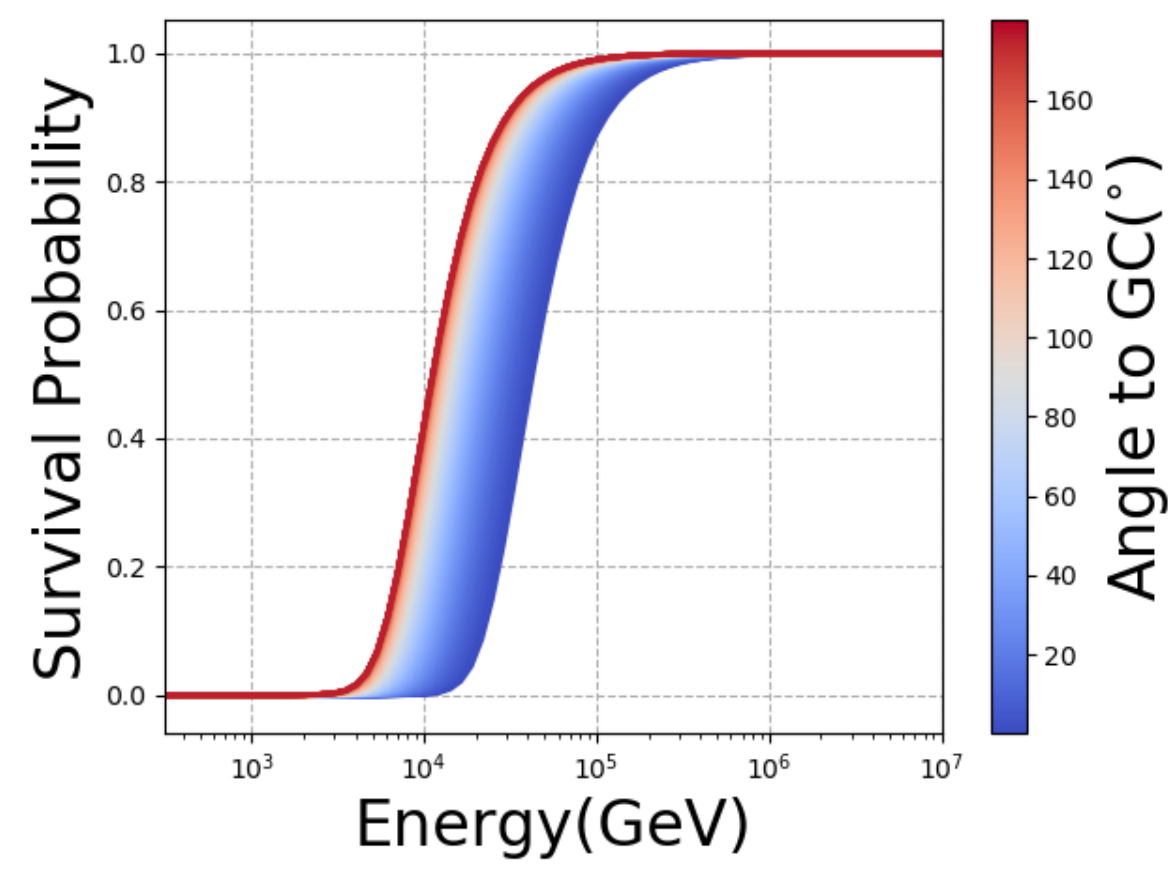

Figure 5.7: Survival probability for a neutrino flux across energy and angle to the Galactic Centre. This is for the scalar mediator-scalar DM scenario with $g=1, m_{\phi}=\mathrm{MeV}, m_{\chi}=1 \mathrm{GeV}$. The low energy $(\leq 20 \mathrm{TeV})$ part of the neutrino flux is completely attenuated by DM-neutrino scatter, while the high energy $(\leq 500 \mathrm{TeV})$ is unaffected. The inclusion of energies down to $1 \mathrm{TeV}$ by MESE-C compared to the $30 \mathrm{TeV}$ of HESE demonstrates how MESE-C will be able to better probe certain DM-neutrino scattering interactions. 


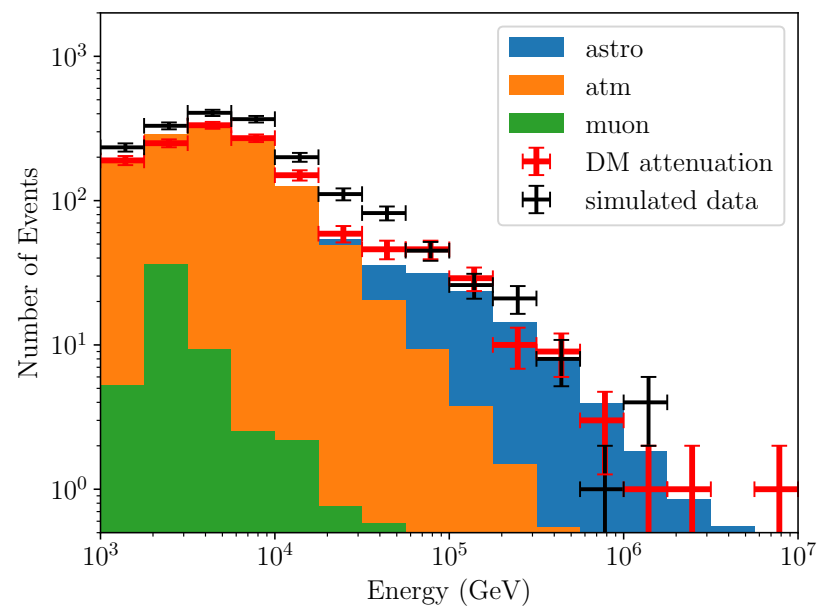

Figure 5.8: The energy spectrum that includes a DM attenuation of the astrophysical neutrino flux.

\subsection{Likelihood}

For this analysis, an unbinned likelihood method is used to compare the expected events with detected events at IceCube for a scalar DM-scalar mediator model. The likelihood function was composed of hypothesis parameters for the DM contribution: $\vec{\theta}=\left\{g, m_{\phi}, m_{\chi}\right\}$, nuisance parameters for modeling the background: $\vec{\eta}=$ $\left\{\gamma, \phi_{\text {astro }}, \Delta \gamma, \phi_{\text {atm }}, \phi_{\text {muon }}\right\}$ and observable parameters as reconstructed at IceCube: $\vec{x}_{\text {reco }}=\left\{\log _{10}\left(E_{\nu}\right), \alpha, \sin (\delta)\right\}$. Here $m_{\chi}$ is the DM mass, $m_{\phi}$ is the mediator mass, and $g$ is the coupling strength. For the nuisance parameters $\gamma$ is the spectral index of the astrophysical flux, $\phi_{\text {astro }}$ is the normalization of the astrophysical flux, $\Delta \gamma$ is the spectral hardening/softening parameter for the atmospheric flux, and $\phi_{\text {muon }}$ is the normalization of the muon flux. The observable parameters at IceCube are en-

ergy $\log _{10} E$, right ascension $\alpha$ and the declination $\sin (\delta)$. The likelihood of a DM 


\subsection{LIKELIHOOD}

hypothesis, $\vec{\theta}$, given the data, $\vec{x}$ and including nuisance parameters, $\vec{\eta}$ is:

$$
\mathcal{L}(\vec{\theta}, \vec{\eta} ;\{\vec{x} \in \text { dataset }\})=\frac{e^{-\lambda(\vec{\theta}, \vec{\eta})} \lambda(\vec{\theta}, \vec{\eta})^{k}}{k !} \prod_{i}^{k} f\left(\vec{x}_{i}, \vec{\theta}, \vec{\eta}\right)
$$

The likelihood is composed of a Poisson normalization for the whole dataset that compares the total number of events observed, $k$, to the number of expected events $\lambda(\vec{\theta}, \vec{\eta})$. The second component is a product over the likelihoods for each individual event, $i$, in the dataset. Here the likelihood for each individual event, $f\left(\vec{x}_{i}, \vec{\theta}, \vec{\eta}\right)$, includes shape effects across the physical observables $\vec{x}=\left\{E_{\nu}, \alpha, \delta\right\}$ as well the weights to account for the detector properties:

$$
f\left(\vec{x}_{i}, \vec{\theta}, \vec{\eta}\right)=\frac{N^{\text {astro }} P_{i}^{\text {astro }}+N^{\text {atm }} P_{i}^{a t m}+N^{\text {muon }} P_{i}^{\text {muon }}}{N^{\text {astro }}+N^{\text {atm }}+N^{\text {muon }}},
$$

where $N^{\text {astro }}, N^{\text {atm }}$, and $N^{\text {muon }}$ are the number of expected astrophysical neutrinos, atmospheric neutrinos, and atmospheric muons respectively and $P_{i}^{\text {astro }}, P_{i}^{a t m}$, and $P_{i}^{\text {muon }}$ are the probability distributions for an individual event; these numbers are determined from the flux assumptions of the hypothesis. It should be noted that each probability, $P$ and number of events $N$ is a function of $\left(\vec{x}_{i}, \vec{\theta}, \vec{\eta}\right)$.

The number of expected events are found by integrating the weighted flux across energy, $E$ :

$$
N\left(\vec{x}_{i}, \vec{\theta}, \vec{\eta}\right)=\int \frac{d^{3} \Phi}{d E d \Omega}\left(E_{j}, \alpha_{j}, \delta_{j} ; \vec{\theta}, \vec{\eta}_{j}\right) \frac{L}{g\left(\vec{\eta}_{j}\right)} d E
$$

where $\frac{d^{3} \Phi}{d E d \Omega}\left(E_{j}, \alpha_{j}, \delta_{j} ; \vec{\theta}, \vec{\eta}_{j}\right)$ is the neutrino flux associated with each source and $\frac{L}{g\left(\vec{\eta}_{j}\right)}$ represents a weight factor that incorporates the detector properties including livetime $L$, and OneWeight, $1 / g(\vec{\eta})$. The OneWeight factor accounts for spectral, direction, oversampling and other biases. 


\subsection{LIKELIHOOD}

The probability $P$ associated with each source is constructed from the Monte Carlo simulated dataset. These probability density functions are evaluated for the observables $\vec{x}_{i}$ for each observed event $i$ :

$$
P\left(\vec{x}_{i}, \vec{\theta}, \vec{\eta}\right)=\sum_{j} K\left(\vec{x}_{i}-\vec{x}_{j}\right) \frac{d \Phi}{d E d \Omega}\left(E_{j}, \alpha_{j}, \delta_{j} ; \vec{\theta}, \overrightarrow{\eta_{j}}\right) \frac{L}{g\left(\vec{\eta}_{j}\right)}
$$

where $K$ is the kernel density estimate function that is used to smooth the probability distribution. This probability function is constructed from the Monte Carlo simulation containing $j$ events. Each of the components of the likelihood is explicitly described below.

\section{Astrophysical}

The DM hypothesis is incorporated as an attenuation on the astrophysical flux term as computed by nuFATE, so that the probability for the astrophysical neutrinos is:

$$
P_{i}^{a s t r o}=\sum_{j} K\left(\vec{x}_{i}-\vec{x}_{j}\right) \frac{d \Phi_{a s t r o}}{d E_{\nu}} \frac{L}{g\left(\vec{\eta}_{j}\right)},
$$

where $\frac{d \Phi_{\text {astro }}}{d E_{\nu}}$ is the astrophysical neutrino flux that contains the DM attenuation from Eq. (5.8), $L$ is the livetime, $1 / g(\vec{\eta})$ is the OneWeight, and $K\left(\vec{x}_{i}-\vec{x}_{j}\right)$ is the kernel density estimate function that is describe in Sec. 5.3.1. 


\subsection{LIKELIHOOD}

\section{Atmospheric}

The atmospheric neutrino flux described in 3.6.2 includes the conventional neutrino flux as calculated with a Honda-Gaisser model [121]:

$$
P_{i}^{a t m}=\sum_{j} K\left(\vec{x}_{i}-\vec{x}_{j}\right) \phi_{a t m} w_{H G} w_{n u V e t o}\left(\frac{E_{\nu}}{2020 \mathrm{GeV}}\right)^{\Delta \gamma} \frac{L}{g\left(\vec{\eta}_{j}\right)},
$$

where $K\left(\vec{x}_{i}-\vec{x}_{j}\right)$ is the kernel density estimate function that is describe in Sec. 5.3.1, $\phi_{\text {atm }}$ is the atmospheric flux normalization, $\Delta \gamma$ is a spectral softening/hardening factor, $L$ is the livetime and $1 / g(\vec{\eta})$ is the OneWeight, $w_{H G}$ is the Honda-Gaisser weight from nuflux, $w_{\text {nuVeto }}$ is the weight from nuVeto.

\section{Muon}

The muon events are computed using the muongun software [122]:

$$
P_{i}^{\text {muon }}=\sum_{j} K\left(\vec{x}_{i}-\vec{x}_{j}\right) \phi_{\text {muon }} w_{\mu} L,
$$

where $K\left(\vec{x}_{i}-\vec{x}_{j}\right)$ is the kernel density estimate function that is describe in Sec. 5.3.1, $\phi_{\text {muon }}$ is the muon normalization, $L$ is the livetime and $w_{\mu}$ is the weighting from muongun.

\subsubsection{Kernel Density Estimation}

Kernel density estimation (KDE) is a method used to obtain a continuous probability density function (PDF) from a discrete set of samples. As binning can result in a loss of statistical information KDEs, allow one to smooth a histogram distribution. This is shown in Fig. 5.9. KDEs were used for the PDFs that describe the shape of the 


\subsection{LIKELIHOOD}

distribution of expected events over energy and direction.
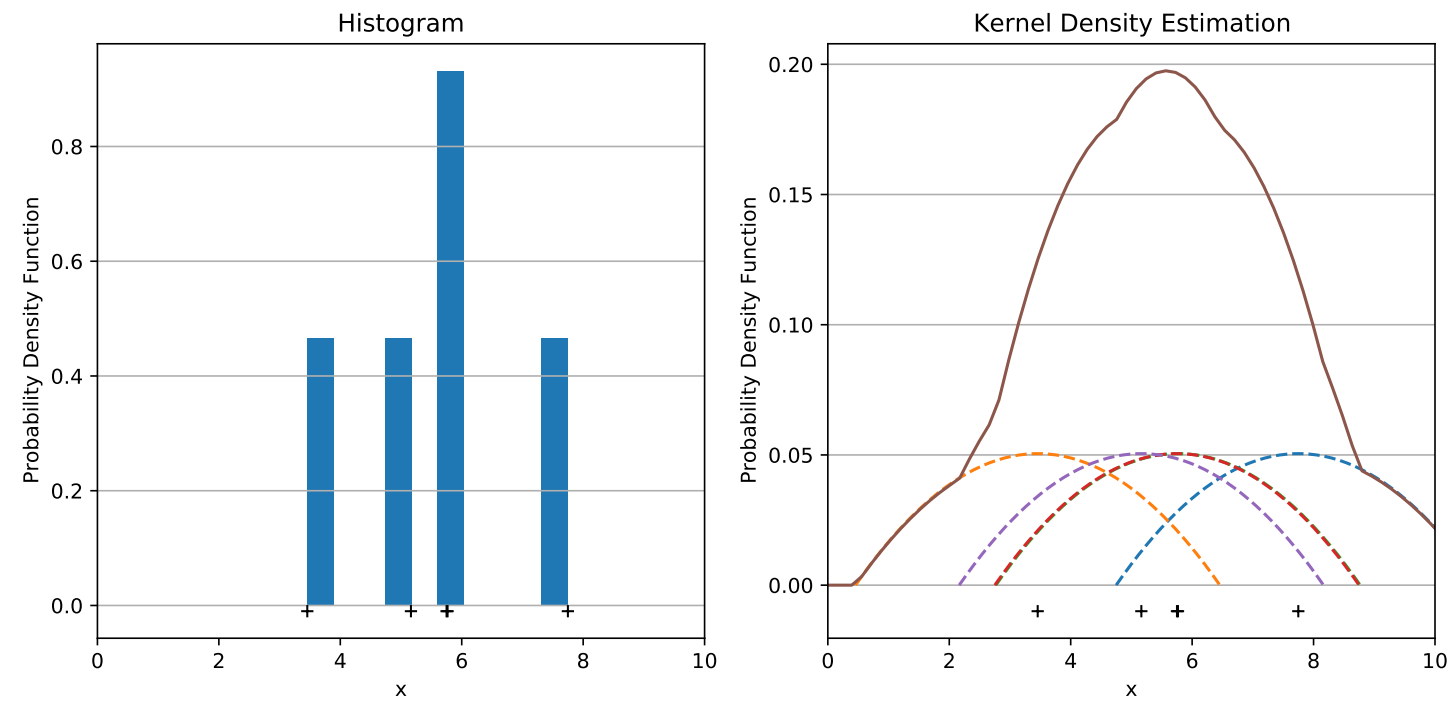

Figure 5.9: A comparison of kernel density estimation (KDE) and histogram binning. KDEs place a kernel at each event location and take the sum of the kernels to determine the overall probability density.

When computing KDEs, a kernel function is placed over each event, $x_{i}$ and the kernels across all events are summed to determine the overall PDF at any point, $x$ in observable space. In one-dimension [128]:

$$
\operatorname{PDF}(x)=\frac{1}{n h} \sum_{i=1}^{n} K\left(\frac{x-x_{i}}{h}\right),
$$

where $n$ is the total number of events, $K(x)$ is the kernel function evaluated at $x$ and $h$ is the bandwidth of the kernel. The PDFs were constructed using the simulated Monte Carlo and evaluated at each data point.

Since the Monte Carlo simulation is weighted by detector parameters, a weighted KDE was used. For weighed KDEs each event in the KDE is multiplied by its weight 
$w_{i}$ :

$$
\operatorname{PDF}(x)=\frac{1}{n h} \sum_{i=1}^{n} w_{i} K\left(\frac{x-x_{i}}{h}\right) .
$$

The kernel function used in this thesis was the Epanechnikov kernel which takes the form [128]:

$$
K(u)=\frac{3}{4}\left(1-u^{2}\right)
$$

with $|u| \leq 1$. It can be shown that the Epanichnikov is the optimal kernel with the least mean square error [128].

When extended to multiple dimensions the KDE takes the form [128]:

$$
\operatorname{PDF}(\vec{x})=\frac{1}{n|\vec{H}|^{1 / 2}} \sum_{i=1}^{n} K\left(\frac{\left|\vec{x}-\vec{x}_{i}\right|}{|\vec{H}|^{1 / 2}}\right)
$$

where $n$ is the total number of events, $K(\vec{x})$ is the kernel evaluated at $\vec{x}$ and $\vec{H}$ is the bandwidth matrix.

The 3-D Epanechnikov can be represented as [128]:

$$
K(\vec{u})=\frac{3 \cdot 15}{8 \pi}\left(1-|\vec{u}|^{2}\right)
$$

Since the Monte Carlo simulated data is large, this marked a computationally expensive part of the likelihood. As such, the KDE was precomputed, stored in memory and reweighted for each likelihood evaluation. The KDE was implemented with Scikit-learn using the method prescribed by Langren and Warin [128].

A further treatment of the angular probability distribution could be performed 


\subsection{LIKELIHOOD}

by treating the directional coordinates on a spherical surface with the von MisesFisher distribution [129], which is to a sphere what the Gaussian distribution is to a line. Alternatively, the directional parameters could be reduced to a single angle representing the angular distance to the Galactic Centre.

Sample plots of the KDEs that describe the probability density functions for the energy, right ascension and declination are shown in Fig. 5.10.

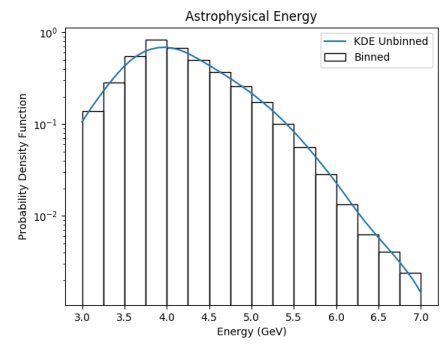

(a) Astrophysical energy

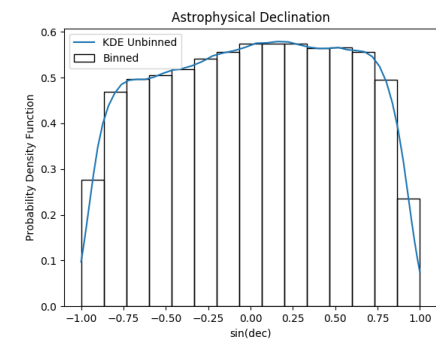

(b) Astrophysical declination

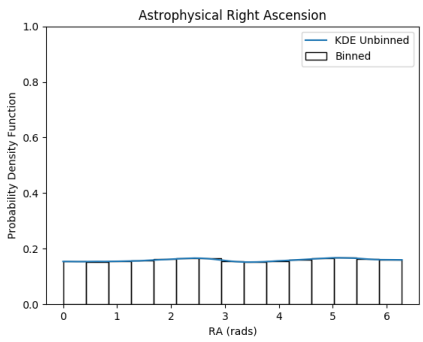

(c) Astrophysical right ascension

Figure 5.10: Probability density functions for the energy, declination and right ascension that are fitted with a KDE. These are used to account for shape effects in the data.

To properly model these probabilities with KDEs, an appropriate bandwidth needs to be selected. The variations in the PDF that result from different bandwidths are shown in Fig. 5.11. 


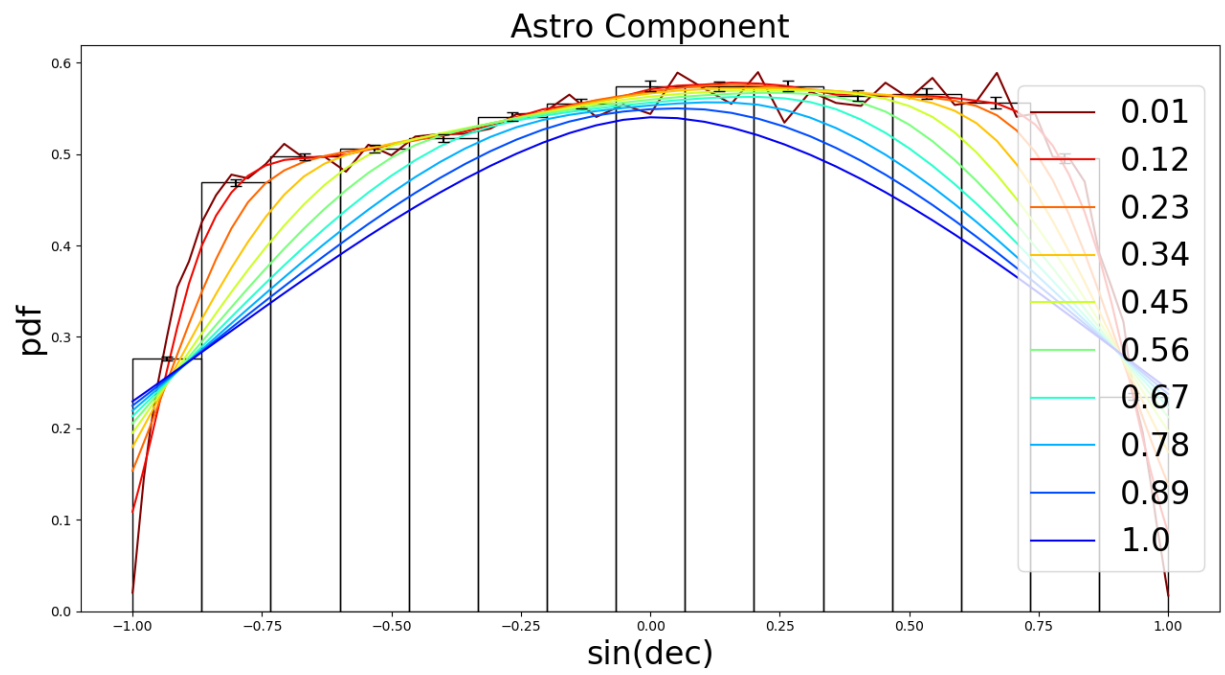

Figure 5.11: Different bandwidths and their effects on the resultant PDF. In this case the ideal bandwidth was about 0.1 and was determined using a chi squared figure of merit.

\subsection{Markov Chain Monte Carlo}

The method that was used to determine sensitivities was a Markov Chain Monte Carlo (MCMC) sampler to constrain DM parameters with the python module emcee [130]. MCMC methods are an efficient way to explore the posterior probability across a large probability space. In fact, while conventional likelihood grid scans scale as $k^{N}$ for $N$ dimensions and $k$ points, MCMC methods scale as $\sim N$. MCMC methods also allow for the inclusion of nuisance parameters [131].

The posterior probability that is explored with the MCMC is expressed with Bayes rule as $[4,132]$ :

$$
P(\theta \mid d)=\frac{P(d \mid \theta) P(\theta)}{P(d)},
$$

for a theory $\theta$ and dataset $d$. $P(\theta \mid d)$ is the posterior probability, $P(d \mid \theta)$ the likelihood from Eq. (5.9), $P(\theta)$ is the prior, and $P(d)$ is the marginal likelihood. Uniform priors 


\subsection{MARKOV CHAIN MONTE CARLO}

over $\left\{\gamma, \phi_{\text {astro }}, \phi_{a t m}, \delta \gamma, \log m_{\chi}, \log m_{\phi}, \log \left(g_{\nu} g_{\chi}\right)\right\}$ are used. While the marginal likelihood is typically difficult to compute, MCMC methods like emcee allow this term to be neglected. emcee makes use of an affine-invariant ensemble sampling algorithm incorporating multiple 'walkers' that sample the parameter space. The algorithm for a single walker $X_{k}$ is $[130,133]$ :

1. Randomly select a walker, $X_{j}$, from the set of all other walkers

2. Form a probability distribution based on the distance between the walkers $X_{j}$ and $X_{k}$. This distribution is given as $Z=\left(X_{k}-X_{j}\right)^{-1 / 2}$.

3. Select a new candidate position $X_{j}+Z$.

4. Evaluate the posterior at the new location, $p\left(X_{k}\right)$ and accept this location if it has a probability greater than $Z^{1-N} p\left(X_{k}\right) . N$ is the number of dimensions.

5. If the new point is accepted, add this new point to the chain and repeat the process.

The chain of points that have been sampled by an MCMC represent the posterior probability distribution in the limit that a large number of points have been sampled. This posterior distribution can then be used to set limits on the DM parameters by marginalizing over nuisance parameters [132]:

$$
P(\theta \mid d)=\int P(\theta, \eta \mid d) d \eta
$$

For the case of the MCMC chain the posterior probability density is obtained by constructing a histogram or KDE across the parameter of interest. 


\subsection{MARKOV CHAIN MONTE CARLO}

In the case of uninformative (uniform) priors the likelihood and posterior distri-

bution are proportional: $P(\theta \mid d) \propto P(d \mid \theta)$, in which case the profile likelihood test statistic can also be used as:

$$
T S=-2 \ln \left(\frac{\mathcal{L}(\theta)}{\mathcal{L}(\prime)}\right)
$$




\section{Chapter 6}

\section{Sensitivity Analysis}

As part of this analysis the sensitivity of IceCube to a scalar DM-scalar mediator model are explored with a sample Monte Carlo dataset using the method described in Sec. 5. Such a search has not been performed for this model in the literature at high energies, but the sensitivities can compared with the low energy cosmology constraints.

\subsection{Nuisance parameters}

The nuisance parameters in this analysis were included to account for variations in the modeling of background events. These nuisance parameters included the atmospheric neutrino and muon backgrounds as well as the isotropic astrophysical flux. To confirm that the method would recover the null hypothesis when the DM hypothesis was not included, the posteriors for the nuisance parameters were determined. These are shown in Fig. 6.1. Here the best fit values for the neutrino flux are $\gamma=-2.7_{-0.05}^{+0.06}$, $\phi_{\text {astro }}=2.18_{-0.21}^{+0.20}, \phi_{\text {atm }}=0.96_{-0.04}^{+0.05}, \phi_{\text {muon }}=1.08_{-0.26}^{+0.28}$ and $\Delta \gamma=-0.11_{-0.04}^{+0.04}$.

These recovered spectral index and flux normalizations were consistent with previously reported values $[13,66,134-138]$, indicating that the models used to describe 


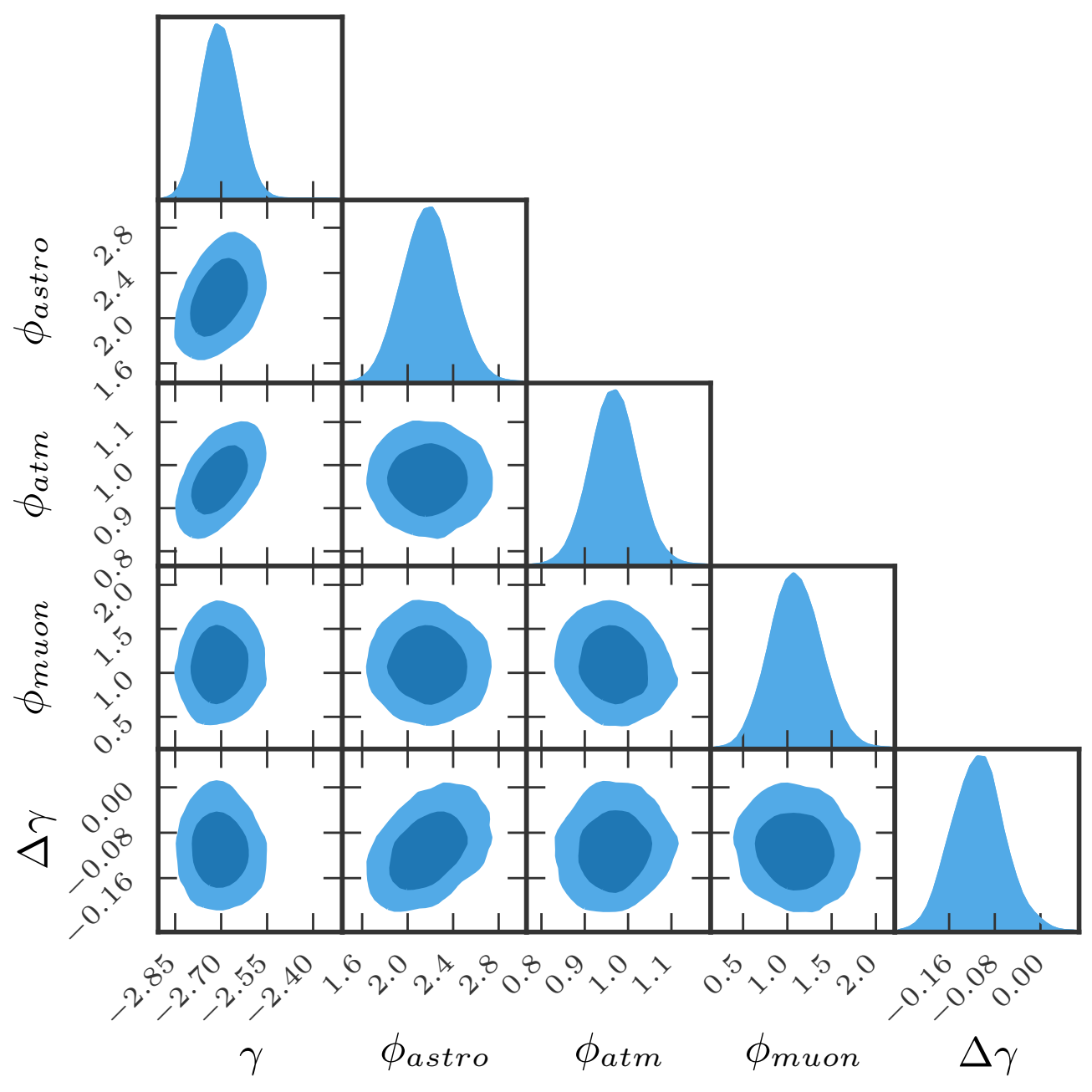

Figure 6.1: Posterior probabilities as sampled by emcee for the no DM scenario. This was to ensure that reasonable values for the spectral indices and normalizations of the background models. 


\subsection{SCALAR DM-SCALAR MEDIATOR}

the neutrino background sources in Sec 3.6 are reasonable.

\subsection{Scalar DM-Scalar Mediator}

Here the case of a scalar DM and scalar mediator is considered to determine sensitivities on the neutrino DM interaction as observed at IceCube. The marginalized posterior probabilities from emcee are shown in Fig. 6.2. The coloured areas signify allowed parameter values, with the dark shading for $95 \%$ credible regions and the light shading for $68 \%$ credible regions. The preference for low mass mediators is found with high mediator masses being excluded. The DM mass is also found to prefer high DM masses up to about $\mathrm{GeV}$, where the posterior drops off. This is expected for a light scalar DM scenario [139].

The best fit values from emcee can be mapped onto the expected event distribution at IceCube as shown with the banded line in Fig. 6.3, here the coloured bars and black crosses correspond to a no DM scenario. These results are consistent with the null hypothesis indicating that a signal from DM-neutrino wasn't found above certain

cross sections. As such, sensitives for the upper limits that can be achieved with the MESE-C dataset can be obtained. This is done by both setting an energy and DM mass dependent upper limit on the scattering cross section as well as exploring the maximum coupling strength for such an interaction. 


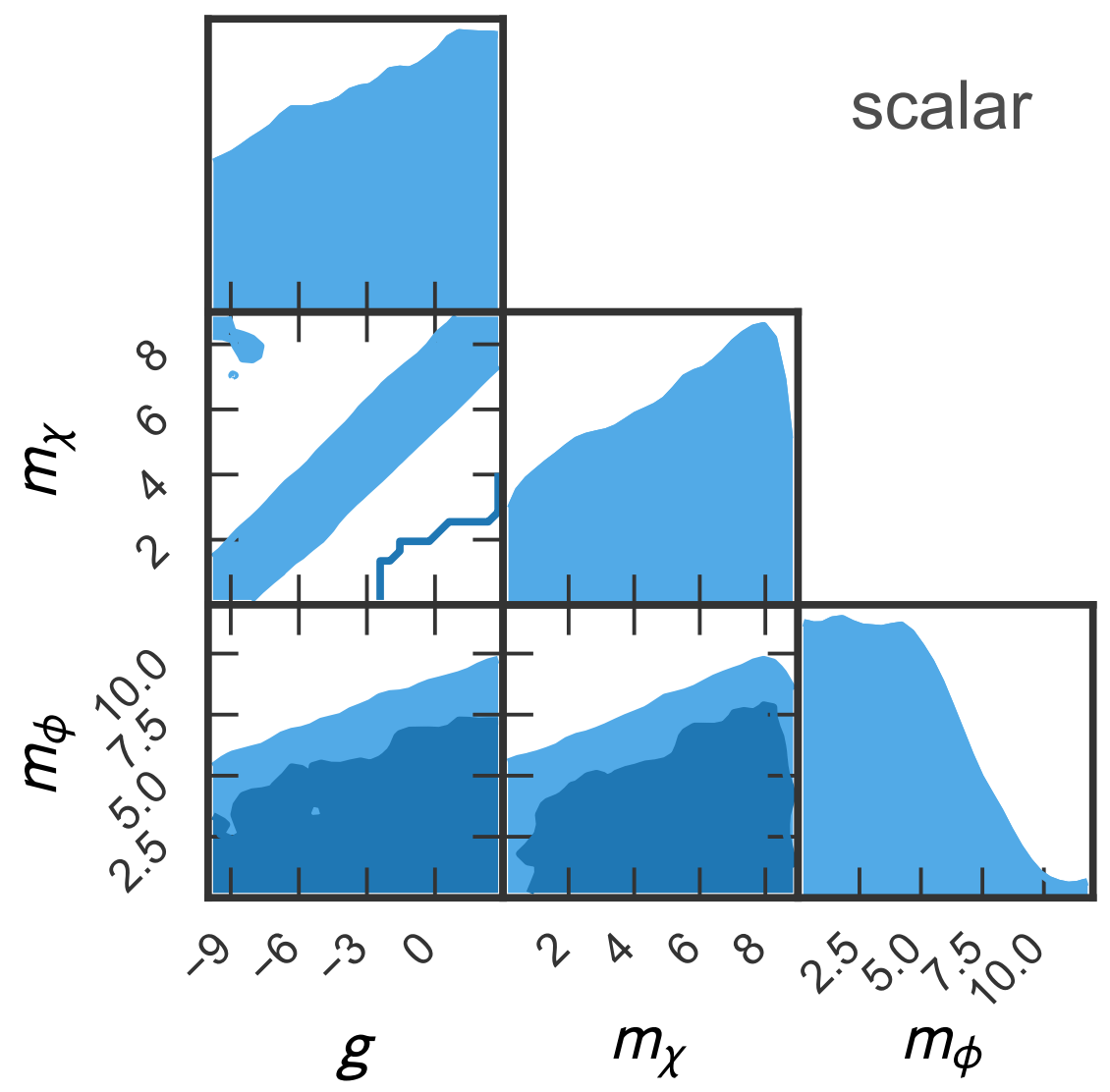

Figure 6.2: The posterior probability distribution for the DM parameters as sampled by emcee.
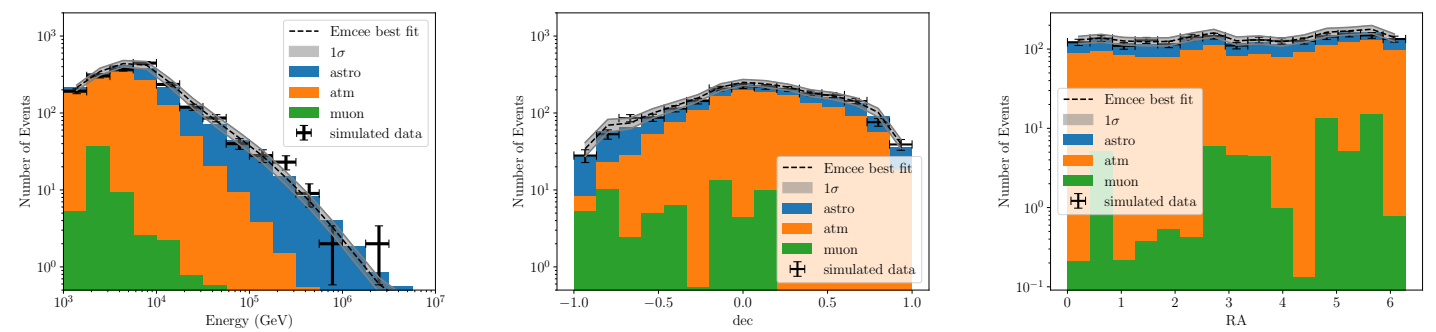

Figure 6.3: The best fit values from the posterior distribution that are expected to be observed at IceCube. These are consistent with a null hypothesis.

The maximum coupling constant that is allowed under the simulated dataset is 


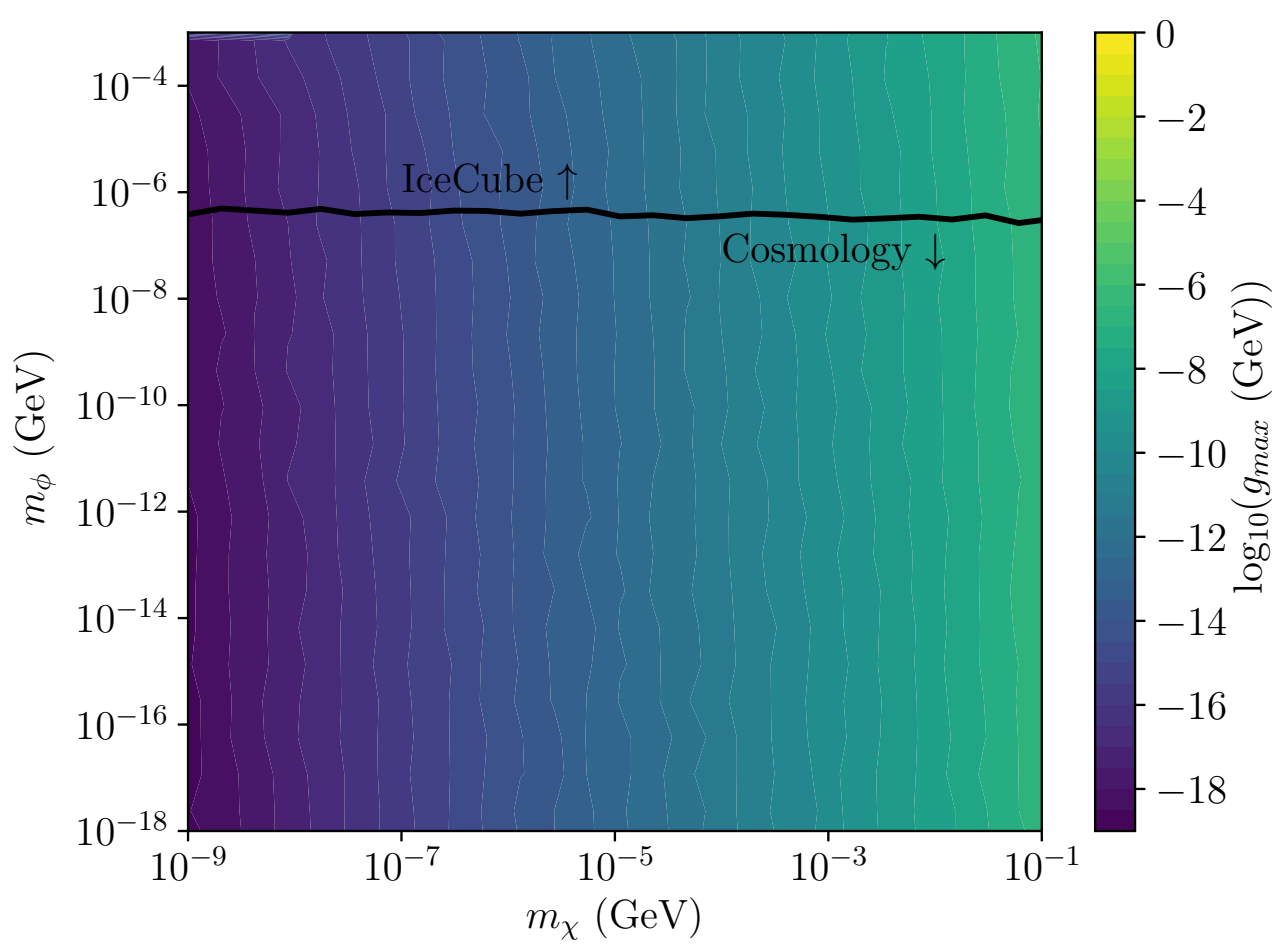

Figure 6.4: The maximum coupling constant that is expected to be allowed by IceCube. Above the line constraints from IceCube are more stringent, while below the line those from cosmology dominate.

plotted in Fig 6.4. Above the line IceCube is more sensitive, while below the line cosmology is more sensitive to constraining the coupling constant. It can be seen that across a range of DM masses and for high mediator masses $\left(m \phi \gtrsim 10^{-6}\right)$, IceCube is more sensitive in constraining the maximum coupling for a DM-neutrino scattering scenario. Previous work with the HESE dataset presented a similar plot for a upper limit on the coupling strength, these were for different models so a direct comparison is not possible. Future work should consider setting constraints with the same models used in the HESE DM-neutrino scattering analysis so a comparison can be made.

The cross section limits from IceCube can be plotted by marginalizing over the 


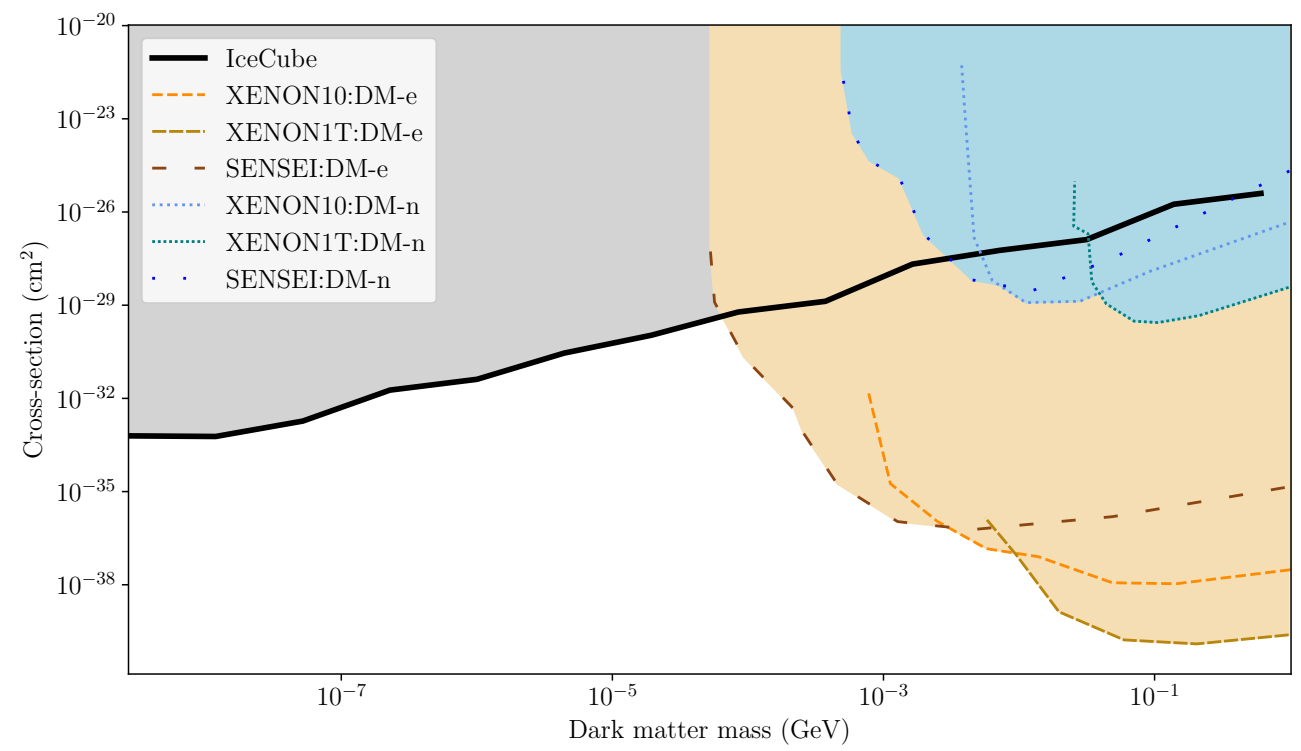

Figure 6.5: The upper limit on the sensitivity for a neutrino-DM cross section is shown in black across DM mass. Also plotted are the DM-electron and DM-nucleon scattering cross section limits from SENSEI, XENON1T and XENON10 as presented in Ref: [16]. Since the cross section is energy dependent, this is plotted for a neutrino energy of $46 \mathrm{TeV}$.

nuisance parameters and making the appropriate transformations on the prior space. The sensitivities obtained are shown in Fig. 6.5, where the upper limit that is expected at IceCube is shown by the black line. The constraints on DM-electron and DMnucleon scattering cross section from the SENSEI and XENON experiments are also shown by the yellow and blue regions respectively for a comparison [16]. This leads to sensitivities at IceCube being set as:

$$
\sigma_{\nu-D M} \lesssim 10^{-27}\left(\frac{m_{\chi}}{\mathrm{GeV}}\right)\left(\frac{E_{\nu}}{\mathrm{PeV}}\right)^{-2} \mathrm{~cm}^{2}
$$

For this particular scalar DM-scalar mediator model these sensitivities mark the first 


\subsection{SCALAR DM-SCALAR MEDIATOR}

limits that can be placed at high neutrino energies. The sensitivity of IceCube was found to be similar to that of cosmology, however, in the high mediator mass range, IceCube can provide stronger constraints on a possible DM-neutrino coupling. This analysis provides a path forward to unblind real IceCube data and set constraints on the DM-neutrino interaction. 


\section{Chapter 7}

\section{Conclusions and Future Work}

\subsection{Conclusion}

The nature of dark matter is one of the greatest unsolved problems in physics. While its gravitational effects have been observed, DM has yet to be detected. In this thesis the elastic scattering interaction between dark matter and neutrinos was explored. These interactions would be expected to occur in areas where neutrinos pass through large column densities of dark matter, such as in the dark matter halo at the centre of Milky Way. This would lead to an attenuation of the extragalactic flux that could be observed at the IceCube Neutrino Observatory. In this thesis software was developed to perform a likelihood analysis of the DM-neutrino scattering interaction. This code incorporated models for backgrounds observed at IceCube, models for the DM halo of the Milky Way and models for the new physics of a DM-neutrino scattering interaction. Through Bayesian inference using Monte Carlo simulations of the IceCube MESE-C dataset sensitivities were set on the dark matter-neutrino scattering cross section. The sensitivity for the DM-neutrino cross section of a scalar

dark matter-scalar mediator model was set as: $\sigma_{\nu-D M} \lesssim 10^{-27}\left(\frac{m_{\chi}}{\mathrm{GeV}}\right)\left(\frac{E_{\nu}}{\mathrm{PeV}}\right)^{-2} \mathrm{~cm}^{2}$. 


\subsection{FUTURE WORK}

This marks the first limits that are set at the high neutrino energies observed in IceCube for the scalar-scalar model. Furthermore, it was shown that IceCube is more sensitive than cosmology in setting constraints on the coupling strength for dark matter-neutrino scattering for certain regions of the parameter space.

\section{$7.2 \quad$ Future Work}

There are numerous avenues to continue this work: exploring additional scattering models, improving the data availability, and incorporating more advanced techniques to account for background neutrino signals and dark matter halo uncertainties.

The other DM-neutrino scattering interaction models described in Sec. 4.3.4 should be constrained. By exploring models that have previously been explored a direct comparison of the limits can be made between the MESE-C and HESE datasets. Future work could also explore other models that have been studied in the literature $[100,140,141]$. Future work should also perform and 'inject and recovery' of a dark matter signal in the data. This involves injecting a fake dark matter signal into the MESE-C data, and verifying that the likelihood sampler would be able to recover the correct DM parameters.

While this thesis considered Monte Carlo simulations to determine sensitivities

for the MESE-C dataset, constraints from an unblinded dataset will be set following the method described in Sec. 5 in a future paper. This unblinding procedure involves performing various validations of the analysis technique and obtaining approvals from the IceCube collaboration to perform the analysis on the full dataset. Future analyses could also expand the present dataset to include track events, use other datasets like the HESE 7.5 year sample (which has 100 astrophysical events) and make use of other 


\subsection{FUTURE WORK}

neutrino observatories. Neutrino observatories located in the northern hemisphere would allow for a simpler veto technique to be used, with the Earth as a shield against the muon background. Future detectors that could provide improved bounds include P-ONE [142], ANTARES [143], KM3NeT [144], IceCube Gen2 [145], and Lake Baikal GVD [146].

Due to the complexity and computational intensity of a multidimensional parameter exploration, this analysis considered a limited number of variables to model the nuisance parameters and dark matter interaction terms. Future work could consider more nuisance parameters such as dark matter halo parameters, or a more advanced background model. For instance a double power law in the astrophysical neutrino flux could be implemented similar to the HESE 7.5 year analysis [13]. As the DM halo parameters have large uncertainties (especially the scale radius) these should also be included as nuisance parameters [40]. Properly accounting for errors in the bandwidth on the KDE should also be included to avoid overfitting. Incorporating more nuisance parameters would be computationally intensive and other Bayesian techniques could be considered such as Gibb's or nested sampling [132]. A more thorough search could consider possible points sources of dark matter such as nearby dwarf galaxies. A more thorough search could also include the extragalactic DM-neutrino interactions as was done in Ref: [107]. 


\section{BIBLIOGRAPHY}

\section{Bibliography}

[1] C. A. Argüelles, A. Kheirandish and A. C. Vincent, Imaging galactic dark matter with high-energy cosmic neutrinos, Phys. Rev. Lett. 119 (2017) 201801.

[2] M. G. Aartsen, M. Ackermann, J. Adams, J. A. Aguilar, M. Ahlers, M. Ahrens et al., Search for sources of astrophysical neutrinos using seven years of icecube cascade events, The Astrophysical Journal 886 (2019) 12.

[3] V. C. Rubin, W. K. Ford, Jr. and N. Thonnard, Extended rotation curves of high-luminosity spiral galaxies. IV. Systematic dynamical properties, Sa through Sc, Astrophys. J. Lett. 225 (1978) L107.

[4] Particle Data Group collaboration, Review of Particle Physics, PTEP 2020 (2020) 083C01.

[5] L. Roszkowski, E. M. Sessolo and S. Trojanowski, Wimp dark matter candidates and searches-current status and future prospects, Reports on Progress in Physics 81 (2018) 066201.

[6] A. Massari, E. Izaguirre, R. Essig, A. Albert, E. Bloom and G. A. Gómez-Vargas, Strong optimized conservative fermi-lat constraints on dark 


\section{BIBLIOGRAPHY}

matter models from the inclusive photon spectrum, Physical Review D 91 (2015) .

[7] E. Vitagliano, I. Tamborra and G. Raffelt, Grand Unified Neutrino Spectrum at Earth: Sources and Spectral Components, Rev. Mod. Phys. 92 (2020) 45006 [1910.11878].

[8] R. Abbasi, M. Ackermann, J. Adams, J. A. Aguilar, M. Ahlers, M. Ahrens et al., Measurement of astrophysical tau neutrinos in icecube's high-energy starting events, 2011.03561.

[9] IceCube, "Graphic resources." https://gallery.icecube.wisc.edu/internal/v/GraphicRe/, 2018.

[10] M. Aartsen, M. Ackermann, J. Adams, J. Aguilar, M. Ahlers, M. Ahrens et al., The icecube neutrino observatory: instrumentation and online systems, Journal of Instrumentation 12 (2017) P03012-P03012.

[11] J. Madsen, Ultra-high energy neutrinos, 1901.02528.

[12] M. G. Aartsen, K. Abraham, M. Ackermann, J. Adams, J. A. Aguilar, M. Ahlers et al., Observation and characterization of a cosmic muon neutrino flux from the northern hemisphere using six years of icecube data, The Astrophysical Journal 833 (2016) 3.

[13] R. Abbasi, M. Ackermann, J. Adams, J. A. Aguilar, M. Ahlers, M. Ahrens et al., The icecube high-energy starting event sample: Description and flux characterization with 7.5 years of data, 2011.03545. 


\section{BIBLIOGRAPHY}

[14] J. A. Aguilar, "Summary of neutrino atmospheric fluxes." https://w3.iihe.ac.be/ aguilar/PHYS-467/PA4.html, 2015.

[15] R. J. Wilkinson, C. Bœhm and J. Lesgourgues, Constraining dark matter-neutrino interactions using the cmb and large-scale structure, Journal of Cosmology and Astroparticle Physics 2014 (2014) 011-011.

[16] L. Barak, I. M. Bloch, M. Cababie, G. Cancelo, L. Chaplinsky, F. Chierchie et al., Sensei: Direct-detection results on sub-gev dark matter from a new skipper ccd, Physical Review Letters 125 (2020).

[17] PLAnCK collaboration, Planck 2015 results. XIII. Cosmological parameters, Astron. Astrophys. 594 (2016) A13 [1502.01589].

[18] G. Bertone, D. Hooper and J. Silk, Particle dark matter: Evidence, candidates and constraints, Phys. Rept. 405 (2005) 279 [hep-ph/0404175].

[19] M. G. e. a. Aartsen, Evidence for high-energy extraterrestrial neutrinos at the icecube detector, Science 342 (2013) 1242856-1242856.

[20] M. Aartsen, M. Ackermann, J. Adams, J. A. Aguilar, M. Ahlers, M. Ahrens et al., Multimessenger observations of a flaring blazar coincident with high-energy neutrino icecube-170922a, Science 361 (2018) .

[21] M. Aartsen, M. Ackermann, J. Adams, J. A. Aguilar, M. Ahlers, M. Ahrens et al., Neutrino emission from the direction of the blazar txs $0506+056$ prior to the icecube-170922a alert, Science 361 (2018) 147-151.

[22] M. Ahlers, K. Helbing and C. Pérez de los Heros, Probing particle physics with icecube, The European Physical Journal C 78 (2018) . 


\section{BIBLIOGRAPHY}

[23] G. Bertone and D. Hooper, History of dark matter, Reviews of Modern Physics 90 (2018) .

[24] F. Zwicky, Die Rotverschiebung von extragalaktischen Nebeln, Helvetica Physica Acta 6 (1933) 110.

[25] F. Zwicky, On the Masses of Nebulae and of Clusters of Nebulae, Astrophys. J. 86 (1937) 217.

[26] V. C. Rubin and W. K. Ford, Jr., Rotation of the Andromeda Nebula from a Spectroscopic Survey of Emission Regions, Astrophys. J. 159 (1970) 379.

[27] M. Bucher, Physics of the cosmic microwave background anisotropy, International Journal of Modern Physics D 24 (2015) 1530004.

[28] R. Tojeiro, Analysing Observables in Structure Formation Theories, Ph.D. thesis, University of Edinburgh, September, 2009.

[29] W. Hu and S. Dodelson, Cosmic microwave background anisotropies, Annual Review of Astronomy and Astrophysics 40 (2002) 171-216.

[30] A. L. Coil, The large-scale structure of the universe, Planets, Stars and Stellar Systems (2013) 387-421.

[31] L. Bergström, Dark matter candidates, New Journal of Physics 11 (2009) 105006 .

[32] S. Dodelson and L. M. Widrow, Sterile neutrinos as dark matter, Physical Review Letters 72 (1994) 17-20. 


\section{BIBLIOGRAPHY}

[33] M. Aartsen, R. Abbasi, M. Ackermann, J. Adams, J. Aguilar, M. Ahlers et al., ev-scale sterile neutrino search using eight years of atmospheric muon neutrino data from the icecube neutrino observatory, Physical Review Letters $125(2020)$.

[34] M. Aartsen, M. Ackermann, J. Adams, J. Aguilar, M. Ahlers, M. Ahrens et al., Search for sterile neutrino mixing using three years of icecube deepcore data, Physical Review D 95 (2017) .

[35] J. An and H. Zhao, Fitting functions for dark matter density profiles, Monthly Notices of the Royal Astronomical Society 428 (2012) 2805-2811.

[36] J. F. Navarro, C. S. Frenk and S. D. M. White, A universal density profile from hierarchical clustering, The Astrophysical Journal 490 (1997) 493-508.

[37] A. Burkert, The structure of dark matter halos in dwarf galaxies, The Astrophysical Journal 447 (1995) .

[38] J. Einasto, On the Construction of a Composite Model for the Galaxy and on the Determination of the System of Galactic Parameters, Trudy Astrofizicheskogo Instituta Alma-Ata 5 (1965) 87.

[39] M. Benito, A. Cuoco and F. Iocco, Handling the uncertainties in the galactic dark matter distribution for particle dark matter searches, Journal of Cosmology and Astroparticle Physics 2019 (2019) 033-033.

[40] M. Benito, F. Iocco and A. Cuoco, Uncertainties in the galactic dark matter distribution: an update, 2009.13523. 


\section{BIBLIOGRAPHY}

[41] C. A. Argüelles, M. Bustamante, A. Kheirandish, S. Palomares-Ruiz, J. Salvado and A. C. Vincent, Fundamental physics with high-energy cosmic neutrinos today and in the future, 1907.08690.

[42] A. J. Lowe, Neutrino physics \&3 the solar neutrino problem, 0907.3658.

[43] N. Aghanim, Y. Akrami, M. Ashdown, J. Aumont, C. Baccigalupi, M. Ballardini et al., Planck 2018 results, Astronomy $\&$ Astrophysics 641 (2020) A6.

[44] SNO Collaboration collaboration, Measurement of the rate of $\nu_{e}+d \rightarrow p+p+e^{-}$interactions produced by ${ }^{8} b$ solar neutrinos at the sudbury neutrino observatory, Phys. Rev. Lett. 87 (2001) 071301.

[45] M. Aartsen, M. Ackermann, J. Adams, J. Aguilar, M. Ahlers, M. Ahrens et al., Differential limit on the extremely-high-energy cosmic neutrino flux in the presence of astrophysical background from nine years of icecube data, Physical Review D 98 (2018) .

[46] D. Smith, D. Hooper and A. Vieregg, Revisiting agn as the source of icecube's diffuse neutrino flux, Journal of Cosmology and Astroparticle Physics 2021 (2021) 031.

[47] A. Plavin, Y. Y. Kovalev, Y. A. Kovalev and S. Troitsky, Observational evidence for the origin of high-energy neutrinos in parsec-scale nuclei of radio-bright active galaxies, The Astrophysical Journal 894 (2020) 101.

[48] E. Waxman and J. Bahcall, High energy neutrinos from cosmological gamma-ray burst fireballs, Physical Review Letters 78 (1997) 2292-2295. 


\section{BIBLIOGRAPHY}

[49] M. G. Aartsen, M. Ackermann, J. Adams, J. A. Aguilar, M. Ahlers,

M. Ahrens et al., Search for prompt neutrino emission from gamma-ray bursts with icecube, The Astrophysical Journal 805 (2015) L5.

[50] A. Ambrosone, M. Chianese, D. F. G. Fiorillo, A. Marinelli, G. Miele and O. Pisanti, Starburst galaxies strike back: a multi-messenger analysis with fermi-lat and icecube data, Monthly Notices of the Royal Astronomical Society 503 (2021) 4032-4049.

[51] P. Giommi, T. Glauch, P. Padovani, E. Resconi, A. Turcati and Y. L. Chang, Dissecting the regions around icecube high-energy neutrinos: growing evidence for the blazar connection, Monthly Notices of the Royal Astronomical Society 497 (2020) 865-878.

[52] A. V. Plavin, Y. Y. Kovalev, Y. A. Kovalev and S. V. Troitsky, Directional association of tev to pev astrophysical neutrinos with radio blazars, The Astrophysical Journal 908 (2021) 157.

[53] M. Aartsen, M. Ackermann, J. Adams, J. Aguilar, M. Ahlers, M. Ahrens et al., Time-integrated neutrino source searches with 10 years of icecube data, Physical Review Letters 124 (2020) .

[54] IceCube collaboration, IceCube Data for Neutrino Point-Source Searches Years 2008-2018, 2101.09836.

[55] A. Kheirandish, Identifying galactic sources of high-energy neutrinos, Astrophysics and Space Science $\mathbf{3 6 5}$ (2020) . 


\section{BIBLIOGRAPHY}

[56] F. Capel, D. Mortlock and C. Finley, Bayesian constraints on the astrophysical neutrino source population from icecube data, Physical Review D 101 (2020) .

[57] C. Deaconu, L. Batten, P. Allison, O. Banerjee, J. Beatty, K. Belov et al., A search for ultrahigh-energy neutrinos associated with astrophysical sources using the third flight of anita, Journal of Cosmology and Astroparticle Physics 2021 (2021) 017.

[58] X. Rodrigues, S. Garrappa, S. Gao, V. S. Paliya, A. Franckowiak and W. Winter, Multi-wavelength and neutrino emission from blazar pks $1502+106,2009.04026$.

[59] A. Kochocki, V. Takhistov, A. Kusenko and N. Whitehorn, Contribution of secondary neutrinos from line-of-sight cosmic ray interactions to the icecube diffuse astrophysical flux, 2012.05955.

[60] I. Collaboration, R. Abbasi, M. Ackermann, J. Adams, J. A. Aguilar, M. Ahlers et al., A search for time-dependent astrophysical neutrino emission with icecube data from 2012 to 2017, 2012.01079.

[61] R. Abbasi, M. Ackermann, J. Adams, J. A. Aguilar, M. Ahlers, M. Ahrens et al., Follow-up of astrophysical transients in real time with the icecube neutrino observatory, The Astrophysical Journal 910 (2021) 4.

[62] A. Capanema, A. Esmaili and P. D. Serpico, Where do icecube neutrinos come from? hints from the diffuse gamma-ray flux, Journal of Cosmology and Astroparticle Physics 2021 (2021) 037-037. 


\section{BIBLIOGRAPHY}

[63] S. Sahu, C. E. López Fortín and S. Nagataki, Photohadronic model for the neutrino and gamma-ray emission from txs 0506+056, The Astrophysical Journal 898 (2020) 103.

[64] O. Pisanti, Astrophysical neutrinos: theory, Journal of Physics: Conference Series 1263 (2019) 012004.

[65] T. K. Gaisser, R. Engel and E. Resconi, Cosmic Rays and Particle Physics: 2nd Edition. Cambridge University Press, 6, 2016.

[66] A. C. Vincent, S. Palomares-Ruiz and O. Mena, Analysis of the 4-year icecube high-energy starting events, Physical Review D 94 (2016) .

[67] N. Song, S. W. Li, C. A. Argüelles, M. Bustamante and A. C. Vincent, The future of high-energy astrophysical neutrino flavor measurements, 2012.12893.

[68] C. Giunti, C. Kim and O. U. Press, Fundamentals of Neutrino Physics and Astrophysics. OUP Oxford, 2007.

[69] M. G. Aartsen et al., Measurement of the multi-tev neutrino interaction cross-section with icecube using earth absorption, Nature 551 (2017) 596-600 [1711.08119].

[70] A. C. Vincent, C. A. Argüelles and A. Kheirandish, High-energy neutrino attenuation in the earth and its associated uncertainties, Journal of Cosmology and Astroparticle Physics 2017 (2017) 012-012.

[71] P. J. McMillan, Mass models of the milky way, Monthly Notices of the Royal Astronomical Society 414 (2011) 2446-2457. 


\section{BIBLIOGRAPHY}

[72] M. Ahlers and F. Halzen, Opening a new window onto the universe with icecube, Progress in Particle and Nuclear Physics 102 (2018) 73-88.

[73] P. B. Denton, D. Marfatia and T. J. Weiler, The galactic contribution to icecube's astrophysical neutrino flux, Journal of Cosmology and Astroparticle Physics 2017 (2017) 033-033.

[74] A. Fedynitch, R. Engel, T. K. Gaisser, F. Riehn and T. Stanev, Calculation of conventional and prompt lepton fluxes at very high energy, 1503.00544.

[75] C. Mascaretti and F. Vissani, On the relevance of prompt neutrinos for the interpretation of the icecube signals, Journal of Cosmology and Astroparticle Physics 2019 (2019) 004-004.

[76] M. Honda, T. Kajita, K. Kasahara, S. Midorikawa and T. Sanuki, Calculation of atmospheric neutrino flux using the interaction model calibrated with atmospheric muon data, Physical Review D 75 (2007).

[77] J. van Santen, Neutrino Interactions in IceCube above 1 TeV: Constraints on Atmospheric Charmed-Meson Production and Investigation of the Astrophysical Neutrino Flux with 2 Years of IceCube Data taken 2010-2012, Ph.D. thesis, Wisconsin U., Madison, 11, 2014.

[78] G. Aad, T. Abajyan, B. Abbott, J. Abdallah, S. Abdel Khalek, A. Abdelalim et al., Observation of a new particle in the search for the standard model higgs boson with the atlas detector at the lhc, Physics Letters B 716 (2012) 1-29.

[79] O. Buchmueller, C. Doglioni and L.-T. Wang, Search for dark matter at colliders, Nature Physics 13 (2017) 217-223. 


\section{BIBLIOGRAPHY}

[80] J. L. Feng, Collider Physics and Cosmology, Class. Quant. Grav. 25 (2008) 114003 [0801.1334].

[81] T. Marrodán Undagoitia and L. Rauch, Dark matter direct-detection experiments, J. Phys. G 43 (2016) 013001 [1509.08767].

[82] R. K. Leane, Indirect detection of dark matter in the galaxy, 2006.00513.

[83] J. M. Gaskins, A review of indirect searches for particle dark matter, Contemp. Phys. 57 (2016) 496 [1604.00014].

[84] C. Pérez de los Heros, Status, challenges and directions in indirect dark matter searches, Symmetry 12 (2020) 1648.

[85] A. Lowell, T. Aramaki, R. Bird, M. Boezio, S. Boggs, R. Carr et al., An indirect dark matter search using cosmic-ray antiparticles with gaps, 1812.04800 .

[86] M. G. Aartsen, M. Ackermann, J. Adams, J. A. Aguilar, M. Ahlers, M. Ahrens et al., Multipole analysis of icecube data to search for dark matter accumulated in the galactic halo, The European Physical Journal C 75 (2015) .

[87] A. Albert, M. André, M. Anghinolfi, M. Ardid, J.-J. Aubert, J. Aublin et al., Combined search for neutrinos from dark matter self-annihilation in the galactic center with antares and icecube, Physical Review D 102 (2020) .

[88] M. G. Aartsen, R. Abbasi, Y. Abdou, M. Ackermann, J. Adams, J. A. Aguilar et al., Icecube search for dark matter annihilation in nearby galaxies and galaxy clusters, Physical Review D $8 \mathbf{8}$ (2013) . 


\section{BIBLIOGRAPHY}

[89] M. G. Aartsen, M. Ackermann, J. Adams, J. A. Aguilar, M. Ahlers, M. Ahrens et al., Search for annihilating dark matter in the sun with 3 years of icecube data, The European Physical Journal C 77 (2017) .

[90] M. G. Aartsen, K. Abraham, M. Ackermann, J. Adams, J. A. Aguilar, M. Ahlers et al., First search for dark matter annihilations in the earth with the icecube detector, The European Physical Journal C 77 (2017) .

[91] A. E. Erkoca, M. H. Reno and I. Sarcevic, Probing dark matter models with neutrinos from the galactic center, Physical Review D 82 (2010).

[92] K. C. Y. Ng, A. Dekker, S. Ando, B. Bouwer, M. Geijsen, C. Glazener et al., Sensitivities of km3net on decaying dark matter, 2007.03692.

[93] C. A. Argüelles, A. Diaz, A. Kheirandish, A. Olivares-Del-Campo, I. Safa and A. C. Vincent, Dark matter annihilation to neutrinos, 1912.09486.

[94] M. Chianese, G. Miele and S. Morisi, Dark matter interpretation of low energy icecube mese excess, Journal of Cosmology and Astroparticle Physics 2017 (2017) 007-007.

[95] M. Schumann, Direct detection of wimp dark matter: concepts and status, Journal of Physics G: Nuclear and Particle Physics 46 (2019) 103003.

[96] M. Escudero, L. Lopez-Honorez, O. Mena, S. Palomares-Ruiz and P. Villanueva-Domingo, A fresh look into the interacting dark matter scenario, Journal of Cosmology and Astroparticle Physics 2018 (2018) 007-007.

[97] G. Guo, Y.-L. S. Tsai, M.-R. Wu and Q. Yuan, Elastic and inelastic scattering of cosmic rays on sub-gev dark matter, Physical Review D 102 (2020) . 


\section{BIBLIOGRAPHY}

[98] M. Escudero, O. Mena, A. C. Vincent, R. J. Wilkinson and C. Bœhm, Exploring dark matter microphysics with galaxy surveys, Journal of Cosmology and Astroparticle Physics 2015 (2015) 034-034.

[99] C. Bœhm, M. J. Dolan and C. McCabe, A lower bound on the mass of cold thermal dark matter from planck, Journal of Cosmology and Astroparticle Physics 2013 (2013) 041-041.

[100] A. Olivares-Del Campo, C. Bøhm, S. Palomares-Ruiz and S. Pascoli, Dark matter-neutrino interactions through the lens of their cosmological implications, Physical Review D 97 (2018) .

[101] G. Mangano, A. Melchiorri, P. Serra, A. Cooray and M. Kamionkowski, Cosmological bounds on dark-matter-neutrino interactions, Physical Review D $74(2006)$.

[102] P. Serra, F. Zalamea, A. Cooray, G. Mangano and A. Melchiorri, Constraints on neutrino - dark matter interactions from cosmic microwave background and large scale structure data, 0911.4411.

[103] J. F. Cherry, A. Friedland and I. M. Shoemaker, Neutrino portal dark matter: From dwarf galaxies to icecube, 1411.1071.

[104] B. Bertoni, S. Ipek, D. McKeen and A. E. Nelson, Reducing cosmological small scale structure via a large dark matter-neutrino interaction: constraints and consequences, 1412.3113. 


\section{BIBLIOGRAPHY}

[105] Y. Farzan and S. Palomares-Ruiz, Dips in the diffuse supernova neutrino background, Journal of Cosmology and Astroparticle Physics 2014 (2014) 014-014.

[106] A. Paul, A. Chatterjee, A. Ghoshal and S. Pal, Shedding light on dark matter and neutrino interactions from cosmology, 2104.04760.

[107] J. H. Davis and J. Silk, Spectral and spatial distortions of pev neutrinos from scattering with dark matter, 1505.01843.

[108] A. Das and M. Sen, Boosted dark matter from diffuse supernova neutrinos, 2104.00027.

[109] Y. Jho, J.-C. Park, S. C. Park and P.-Y. Tseng, Cosmic-neutrino-boosted dark matter ( $\nu b d m), 2021$.

[110] S. Koren, Neutrino-dark matter scattering and coincident detections of uhe neutrinos with em sources, Journal of Cosmology and Astroparticle Physics 2019 (2019) 013-013.

[111] M. Ahlers, M. Bustamante and S. Mu, Unitarity bounds of astrophysical neutrinos, Physical Review D 98 (2018) .

[112] C. A. Argüelles, T. Katori and J. Salvado, Effect of new physics in astrophysical neutrino flavor, Physical Review Letters 115 (2015) .

[113] S. Karmakar, S. Pandey and S. Rakshit, Astronomy with energy dependent flavour ratios of extragalactic neutrinos, 2010.07336. 


\section{BIBLIOGRAPHY}

[114] P. de Salas, R. Lineros and M. Tórtola, Neutrino propagation in the galactic dark matter halo, Physical Review D 94 (2016) .

[115] D. W. Hogg, Distance measures in cosmology, arXiv e-prints (1999) astro [astro-ph/9905116].

[116] L. Jiang, N. Kashikawa, S. Wang, G. Walth, L. C. Ho, Z. Cai et al., Evidence for gn-z11 as a luminous galaxy at redshift 10.957, Nature Astronomy $\mathbf{5}$ (2020) 256-261.

[117] M. Gerbino and M. Lattanzi, Status of neutrino properties and future prospects - cosmological and astrophysical constraints, Frontiers in Physics $\mathbf{5}$ (2018) 70 .

[118] J. Lesgourgues and S. Pastor, Neutrino cosmology and Planck, New J. Phys. 16 (2014) 065002 [1404.1740].

[119] J. Lesgourgues and S. Pastor, Neutrino cosmology and planck, New Journal of Physics 16 (2014) 065002.

[120] A. Blaauw, C. S. Gum, J. L. Pawsey and G. Westerhout, The new I. A. U. system of galactic coordinates (1958 revision), Monthly Notices of the Royal Astronomical Society 121 (1960) 123.

[121] R. Abbasi, M. Ackermann, J. Adams, J. A. Aguilar, M. Ahlers, M. Ahrens et al., Leptoninjector and leptonweighter: A neutrino event generator and weighter for neutrino observatories, 2012.10449. 


\section{BIBLIOGRAPHY}

[122] G. Carminati, M. Bazzotti, S. Biagi, S. Cecchini, T. Chiarusi, A. Margiotta et al., Mupage: a fast atmospheric muon generator for neutrino telescopes based on parametric formulas, 0907.5563.

[123] C. A. Argüelles, S. Palomares-Ruiz, A. Schneider, L. Wille and T. Yuan, Unified atmospheric neutrino passing fractions for large-scale neutrino telescopes, Journal of Cosmology and Astroparticle Physics 2018 (2018) 047-047.

[124] M. Aartsen, R. Abbasi, Y. Abdou, M. Ackermann, J. Adams, J. Aguilar et al., Measurement of south pole ice transparency with the icecube led calibration system, Nuclear Instruments and Methods in Physics Research Section A: Accelerators, Spectrometers, Detectors and Associated Equipment 711 (2013) $73-89$.

[125] ICECuBE collaboration, South Pole glacial climate reconstruction from multi-borehole laser particulate stratigraphy, J. Glaciol. 59 (2013) 1117.

[126] IceCube Collaboration, "Weights in nugen simulation data." https://wiki . icecube.wisc.edu/index.php/Weights_in_nugen_Simulation_Data, May, 2017.

[127] IceCube Collaboration, "Neutrino generator/weighting." https: //wiki.icecube.wisc.edu/index.php/Neutrino_Generator/Weighting, Feb., 2020. 


\section{BIBLIOGRAPHY}

[128] N. Langrené and X. Warin, Fast and stable multivariate kernel density estimation by fast sum updating, Journal of Computational and Graphical Statistics 28 (2019) 596-608.

[129] T. Yuan, The 8-parameter fisher-bingham distribution on the sphere, Computational Statistics 36 (2020) 409-420.

[130] D. Foreman-Mackey, D. W. Hogg, D. Lang and J. Goodman, emcee: The mcmc hammer, Publications of the Astronomical Society of the Pacific $\mathbf{1 2 5}$ (2013) 306-312.

[131] L. E. Padilla, L. O. Tellez, L. A. Escamilla and J. A. Vazquez, Cosmological parameter inference with bayesian statistics, 1903.11127.

[132] R. Trotta, Bayesian methods in cosmology, 1701.01467.

[133] J. Goodman and J. Weare, Ensemble samplers with affine invariance, Communications in Applied Mathematics and Computational Science 5 (2010) 65.

[134] S. Palomares-Ruiz, A. C. Vincent and O. Mena, Spectral analysis of the high-energy icecube neutrinos, Physical Review D 91 (2015) .

[135] M. G. Aartsen, K. Abraham, M. Ackermann, J. Adams, J. A. Aguilar, M. Ahlers et al., A combined maximum-likelihood analysis of the high-energy astrophysical neutrino flux measured with icecube, The Astrophysical Journal 809 (2015) 98.

[136] A. Schneider, Characterization of the astrophysical diffuse neutrino flux with icecube high-energy starting events, 1907.11266. 


\section{BIBLIOGRAPHY}

[137] J. Stettner, Measurement of the diffuse astrophysical muon-neutrino spectrum with ten years of icecube data, 1908.09551.

[138] ICECuBE collaboration, A measurement of the diffuse astrophysical muon neutrino flux using eight years of IceCube data., PoS ICRC2017 (2018) 1005.

[139] C. Bøhm and P. Fayet, Scalar dark matter candidates, Nuclear Physics B 683 (2004) 219-263.

[140] J. Stadler, C. Bøehm and O. Mena, Comprehensive study of neutrino-dark matter mixed damping, Journal of Cosmology and Astroparticle Physics 2019 (2019) 014-014.

[141] S. Pandey, S. Karmakar and S. Rakshit, Interactions of astrophysical neutrinos with dark matter: a model building perspective, Journal of High Energy Physics 2019 (2019) .

[142] M. Agostini, M. Böhmer, J. Bosma, K. Clark, M. Danninger, C. Fruck et al., The pacific ocean neutrino experiment, Nature Astronomy 4 (2020) 913-915.

[143] M. Ageron, J. Aguilar, I. Al Samarai, A. Albert, F. Ameli, M. André et al., Antares: The first undersea neutrino telescope, Nuclear Instruments and Methods in Physics Research Section A: Accelerators, Spectrometers, Detectors and Associated Equipment 656 (2011) 11-38.

[144] A. Margiotta, The km3net deep-sea neutrino telescope, Nuclear Instruments and Methods in Physics Research Section A: Accelerators, Spectrometers, Detectors and Associated Equipment 766 (2014) 83-87. 


\section{BIBLIOGRAPHY}

[145] G. Collaboration, M. G. Aartsen, R. Abbasi, M. Ackermann, J. Adams, J. A. Aguilar et al., Icecube-gen2: The window to the extreme universe, 2008.04323.

[146] G. Collaboration, A. D. Avrorin, A. V. Avrorin, V. M. Aynutdinov, R. Bannash, I. A. Belolaptikov et al., Neutrino telescope in lake baikal: Present and future, 1908.05427.

[147] D. J. Griffiths, Introduction to elementary particles; 2nd rev. version. Wiley, New York, NY, 2008.

[148] K. Kumericki, Feynman Diagrams for Beginners, arXiv e-prints (2016) arXiv:1602.04182 [1602.04182]. 


\section{Appendix A}

\section{Cross Sections}

\section{A.1 Matrix elements}

The cross section is derived using the golden rule of scattering as described by Griffiths [147]. A brief summary of Feynman diagram calculations was also referenced here: [148]. Note that $c$ and $\hbar$ terms are omitted in the below calculations and need to be added in so that units will match.

In the lab frame of the dark matter (incoming neutrino, stationary dark matter) the differential cross section is given by [147]:

$$
\frac{d \sigma}{d \Omega}=\left(\frac{E_{\nu_{f}}}{8 \pi m_{\chi} E_{\nu_{i}}}\right)^{2} S|\mathcal{M}|^{2}
$$

where $S=1$ since the products are not identical, $E_{\nu_{i}}$ is the initial energy of the neutrino, $E_{\nu_{f}}$ is the final energy of the neutrino, $m_{\chi}$ is the mass of the dark matter particle, and $\mathcal{M}$ is the matrix element specific to the interaction model. This cross section equation assumes that the neutrino mass is negligible.

The matrix element is constructed following the Feynman rules for the scalarpseudoscalar interaction. The particles are labelled initial neutrino: $p_{1}$, final neutrino: 
$p_{3}$, initial DM: $p_{2}$, final DM: $p_{4}$ as shown in Fig. A.1.

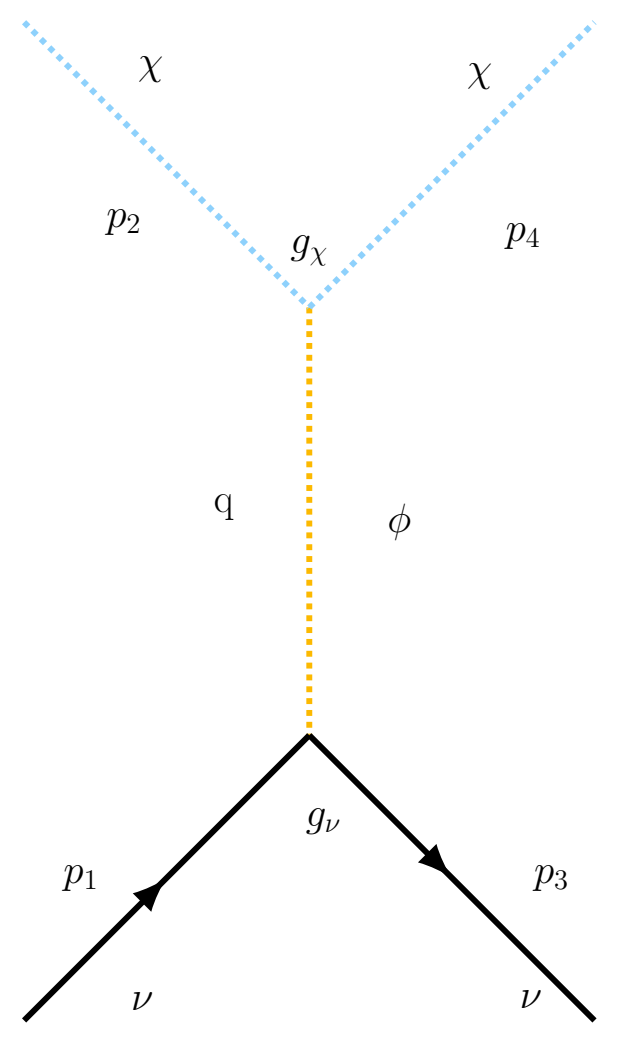

Figure A.1: Feynman diagram of scalar DM-scalar mediator scattering with momenta labels.

The vertex factor between the mediator and neutrinos is $g_{\nu}\left(1+i \gamma^{5}\right)$. The vertex factor between the mediator and dark matter is $g_{\chi}$ and the propagator contributes $\left(\frac{i}{q^{2}-m_{\phi}^{2}}\right)$. The incoming neutrino contributes an external line $u$, while the outgoing neutrino contributes $\bar{u}$. The external lines of the scalar dark matter contribute 1 . The matrix element is thus constructed as:

$$
-i \mathcal{M}=\int\left[\bar{u}\left(p_{3}\right) g_{\nu}\left(i \gamma^{5}+1\right) u\left(p_{1}\right)\right]\left(\frac{i}{q^{2}-m_{\phi}^{2}}\right)\left[(1) i g_{\chi}(1)\right] \delta\left(p_{1}-p_{3}-q\right)(2 \pi)^{4} d q^{4}
$$




\section{A.1. MATRIX ELEMENTS}

Upon integrating over the internal momentum this becomes:

$$
-i \mathcal{M}=\left[\bar{u}\left(p_{3}\right) g_{\nu}\left(i \gamma^{5}+1\right) u\left(p_{1}\right)\right]\left(\frac{g_{\chi}}{\left(p_{1}-p_{3}\right)^{2}-m_{\phi}^{2}}\right)
$$

The absolute value squared is:

$$
|\mathcal{M}|^{2}=\left[\bar{u}\left(p_{3}\right)\left(\gamma^{5}+1\right) u\left(p_{1}\right)\right]\left[\bar{u}\left(p_{3}\right)\left(i \gamma^{5}+1\right) u\left(p_{1}\right)\right]^{*}\left(\frac{g_{\chi} g_{\nu}}{t-m_{\phi}^{2}}\right)^{2}
$$

and using Casimirs trick to sum over spins:

$$
|\mathcal{M}|^{2}=\frac{1}{2} \operatorname{Tr}\left[\left(1+i \gamma^{5}\right) \not p_{1}\left(1-i \gamma^{5}\right) p_{3}\right]\left(\frac{g_{\chi} g_{\nu}}{t-m_{\phi}^{2}}\right)^{2}
$$

With trace commutation relations and noting that $\gamma^{5^{2}}=I$ this becomes:

$$
|\mathcal{M}|^{2}=\operatorname{Tr}\left[\not p_{1}\left(1+\gamma^{5}\right) \not p_{3}\right]\left(\frac{g_{\chi} g_{\nu}}{t-m_{\phi}^{2}}\right)^{2}
$$

These traces were solved using FeynCalc for which the matrix element was found to be:

$$
\sum_{s}|\mathcal{M}|^{2}=g^{2} g^{\prime 2} \frac{-t}{\left(t-m_{\phi}^{2}\right)^{2}}
$$

The outgoing neutrino energy $E_{\nu_{f}}$ is related to the incoming energy $E_{\nu_{i}}$ and scattering angle $\theta$ as:

$$
E_{\nu_{f}}=\left(\frac{1}{E_{\nu_{i}}}+\frac{1}{m_{\chi}}(1-x)\right)^{-1}
$$




\section{A.1. MATRIX ELEMENTS}

where $x=\cos \theta$. In this frame, the Mandelstam variables are:

$$
\begin{aligned}
s & =2 E_{\nu_{i}} m_{\chi}+m_{\chi}^{2}, \\
t & =-2 E_{\nu_{i}} E_{\nu_{f}}(1-x), \\
u & =-2 m_{\chi} E_{\nu_{f}}+m_{\chi}^{2} .
\end{aligned}
$$

Using Eq. (A.1) the differential cross sections for the scalar DM-scalar mediator is:

$$
\frac{d \sigma}{d \cos \theta}=\frac{g_{\nu}^{2} g_{\chi}^{2}(1-x) E_{\nu_{i}}^{2} m_{\chi}}{16 \pi\left((1-x) E_{\nu_{i}}+m_{\chi}\right)\left((1-x) E_{\nu_{i}} m_{\phi}^{2}+m_{\chi}\left(m_{\phi}^{2}-2(x-1) E_{\nu_{i}}^{2}\right)\right)^{2}}
$$

This differential cross section is integrated with Mathematica to determine the total cross section for the scalar DM-scalar mediator as:

$$
\sigma=-g_{\nu}^{2} g_{\chi}^{2} \frac{4 E_{\nu_{i}}^{2} m_{\chi}+\left(2 E_{\nu_{i}} m_{\phi}^{2}+4 E_{\nu_{i}}^{2} m_{\chi}+m_{\phi}^{2} m_{\chi}\right) \log \left(\frac{m_{\phi}^{2}\left(2 E_{\nu_{i}}+m_{\chi}\right)}{2 E_{\nu_{i}} m_{\phi}^{2}+4 E_{\nu_{i}}^{2} m_{\chi}+m_{\phi}^{2} m_{\chi}}\right)}{64 \pi E_{\nu_{i}}^{2} m_{\chi}^{2}\left(2 E_{\nu_{i}} m_{\phi}^{2}+4 E_{\nu_{i}}^{2} m_{\chi}+m_{\phi}^{2} m_{\chi}\right)} .
$$

The cross sections for the other models as described in Ref [1] are given below.

\section{Dirac DM fermion, scalar mediator:}

$$
\frac{d \sigma}{d \cos \theta}=\frac{g_{\nu}^{2} g_{\chi}^{2}(x-1) E_{\nu_{i}}^{2} m_{\chi}^{2}\left(2(x-1) E_{\nu_{i}} m_{\chi}+(x-1) E_{\nu_{i}}^{2}-2 m_{\chi}^{2}\right)}{8 \pi\left(m_{\chi}-(x-1) E_{\nu_{i}}\right)^{2}\left((x-1) E_{\nu_{i}} m_{\phi}^{2}-m_{\chi}\left(m_{\phi}^{2}-2(x-1) E_{\nu_{i}}^{2}\right)\right)^{2}},
$$




\section{A.1. MATRIX ELEMENTS}

and

$$
\begin{aligned}
\sigma= & \frac{g_{\nu}^{2} g_{\chi}^{2}}{32 \pi E_{\nu_{i}}^{2} m_{\chi}^{2}}\left[E_{\nu_{i}} m_{\chi}-\frac{E_{\nu_{i}} m_{\chi}^{2} m_{\phi}^{2}\left(m_{\phi}^{2}-4 m_{\chi}^{2}\right)}{\left(2 E_{\nu_{i}} m_{\chi}+m_{\phi}^{2}\right)\left(4 E_{\nu_{i}}^{2} m_{\chi}+2 E_{\nu_{i}} m_{\phi}^{2}+m_{\chi} m_{\phi}^{2}\right)}(\mathrm{A}\right. \\
& -\frac{E_{\nu_{i}} m_{\chi}^{2}}{2 E_{\nu_{i}}+m_{\chi}}+\frac{E_{\nu_{i}} m_{\chi}\left(m_{\phi}^{2}-4 m_{\chi}^{2}\right)}{2 E_{\nu_{i}} m_{\chi}+m_{\phi}^{2}} \\
& \left.+\left(m_{\phi}^{2}-2 m_{\chi}^{2}\right) \log \left(\frac{m_{\phi}^{2}\left(2 E_{\nu_{i}}+m_{\chi}\right)}{4 E_{\nu_{i}}^{2} m_{\chi}+2 E_{\nu_{i}} m_{\phi}^{2}+m_{\chi} m_{\phi}^{2}}\right)\right]
\end{aligned}
$$

Dirac fermion DM, vector mediator:

$$
\frac{d \sigma}{d \cos \theta}=\frac{g_{\nu}^{2} g_{\chi}^{2} E_{\nu_{i}}^{2} m_{\chi}^{2}\left(\left(1-x^{2}\right) E_{\nu_{i}} m_{\chi}+(1-x)^{2} E_{\nu_{i}}^{2}+(1+x) m_{\chi}^{2}\right)}{4 \pi\left((1-x) E_{\nu_{i}}+m_{\chi}\right)\left((1-x) E_{\nu_{i}} m_{\phi}^{2}+m_{\chi}\left(m_{\phi}^{2}-2(x-1) E_{\nu_{i}}^{2}\right)\right)^{2}}
$$

and

$$
\begin{gathered}
\sigma=\frac{g_{\nu}^{2} g_{\chi}^{2}}{16 \pi E_{\nu_{i}}^{2} m_{\chi}^{2}}\left[\left(m_{\phi}^{2}+m_{\chi}^{2}+2 E_{\nu_{i}} m_{\chi}\right) \log \left(\frac{m_{\phi}^{2}\left(2 E_{\nu_{i}}+m_{\chi}\right)}{m_{\chi}\left(4 E_{\nu_{i}}^{2}+m_{\phi}^{2}\right)+2 E_{\nu_{i}} m_{\phi}^{2}}\right)\right. \\
\left.+4 E_{\nu_{i}}^{2}\left(1+\frac{m_{\chi}^{2}}{m_{\phi}^{2}}-\frac{2 E_{\nu}\left(4 E_{\nu_{i}}^{2} m_{\chi}+E_{\nu_{i}}\left(m_{\chi}^{2}+2 m_{\phi}^{2}\right)+m_{\chi} m_{\phi}^{2}\right.}{\left(2 E_{\nu_{i}}+m_{\chi}\right)\left(m_{\chi}\left(4 E_{\nu_{i}}^{2}+m_{\phi}^{2}\right)+2 E_{\nu_{i}} m_{\phi}^{2}\right)}\right)\right] .
\end{gathered}
$$

Scalar DM, fermion mediator:

$$
\begin{aligned}
\frac{d \sigma}{d \cos \theta} & =\frac{g^{4}(1+x) E_{\nu_{i}}^{4} m_{\chi}^{5}\left((1-x) E_{\nu_{i}}+2 m_{\chi}\right)^{2}}{4 \pi\left(m_{\phi}^{2}-m_{\chi}\left(2 E_{\nu_{i}}+m_{\chi}\right)\right)^{2}\left((1-x) E_{\nu_{i}}+m_{\chi}\right)^{3}} \\
& \times \frac{1}{\left(E_{\nu_{i}}\left((x-1) m_{\phi}^{2}-(x+1) m_{\chi}^{2}\right)+m_{\chi}^{3}-m_{\chi} m_{\phi}^{2}\right)^{2}},
\end{aligned}
$$




\section{A.1. MATRIX ELEMENTS}

and

$$
\begin{aligned}
\sigma & =\frac{g^{4}}{64 \pi}\left[\frac{8 E_{\nu_{i}}^{2} m_{\chi}}{\left(2 E_{\nu_{i}}+m_{\chi}\right)\left(m_{\phi}^{2}-m_{\chi}\left(2 E_{\nu_{i}}+m_{\chi}\right)\right)^{2}}+\frac{4}{-2 E_{\nu_{i}} m_{\chi}+m_{\chi}^{2}-m_{\phi}^{2}}\right. \\
& +\frac{8}{m_{\chi}\left(2 E_{\nu_{i}}+m_{\chi}\right)-m_{\phi}^{2}} \\
& \left.+\left(\frac{4 E_{\nu_{i}} m_{\chi}-2\left(m_{\chi}^{2}+3 m_{\phi}^{2}\right)}{E_{\nu_{i}} m_{\chi}\left(m_{\chi}\left(2 E_{\nu_{i}}+m_{\chi}\right)-m_{\phi}^{2}\right)}+\frac{3}{E_{\nu_{i}}^{2}}\right) \log \left(\frac{4 E_{\nu_{i}}^{2} m_{\chi}}{m_{\phi}^{2}\left(2 E_{\nu_{i}}+m_{\chi}\right)-m_{\chi}^{3}}+1\right)\right]
\end{aligned}
$$

